Check for updates

Cite this: Chem. Soc. Rev., 2021, 50, 2444

Received 31st July 2020

DOI: $10.1039 / \mathrm{d} 0 \mathrm{cs} 00978 \mathrm{~d}$

rsc.li/chem-soc-rev

\section{Molecular and heterogeneous water oxidation catalysts: recent progress and joint perspectives}

\author{
J. Li, (D) C. A. Triana, W. Wan, D. P. Adiyeri Saseendran, (D) Y. Zhao, iD \\ S. E. Balaghi, (D) S. Heidari and G. R. Patzke (D)*
}

\begin{abstract}
The development of reliable water oxidation catalysts (WOCs) is essential for implementing artificial photosynthesis on a large technological scale. WOC research has evolved into two major branches, namely molecular and heterogeneous catalysts. Manifold design principles and plenty of mechanistic insights have been developed in these individual fields after decades of investigations. Over the past years, a growing need for knowledge transfer between both sides has emerged in order to expedite the development and optimization of next-generation WOCs. In this review, we first provide selected recent highlights in the area of molecular WOCs with different nuclearities, together with current mechanistic insight. WOCs offering molecular integrity under operational conditions are ideal platforms for elucidating reaction mechanisms and well-defined structure-function correlations at the atomic level. Next, recent mechanistic advances and design strategies for heterogeneous WOCs are illustrated for representative examples, together with a discussion of their inherent limitations in mechanistic studies. Finally, illustrative cases of knowledge transfer between molecular and heterogeneous WOCs are discussed to highlight the advantages of combining the best of both catalyst types. For the sake of conciseness, this review focuses primarily on WOCs based on the first-row transition metals, which are attracting increasing attention for both fundamental studies and economic applications.
\end{abstract}

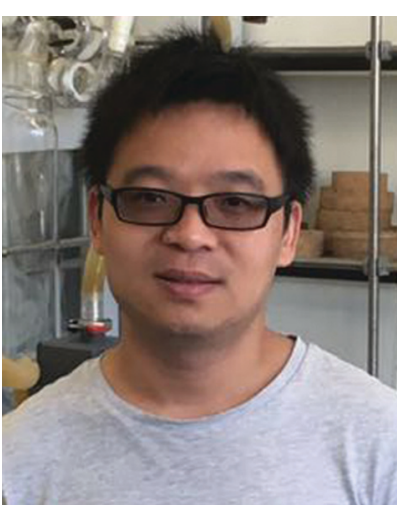

J. Li oxidation research.
Jingguo Li is a PhD student at the University of Zurich in the group of Prof. Greta R. Patzke. He obtained his BSc Degree from Henan University in 2010, and his MSc Degree from the University of Science and Technology of China in 2013. Subsequently, he worked as a Research Engineer at the National University of Singapore, developing polymeric thin films for water purification applications. Then he moved to UZH in 2016 on photo(electrochemical) water

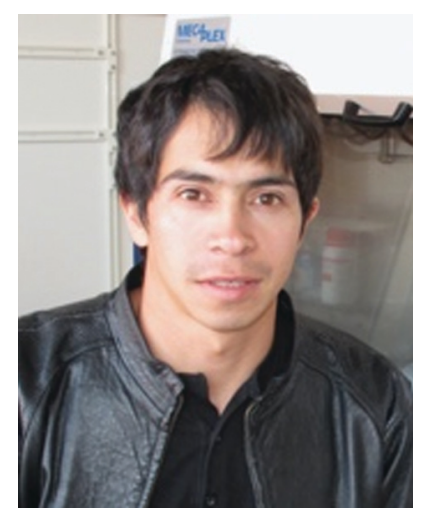

C. A. Triana
Carlos A. Triana received his BSC degree in Physics in 2010 and MSc in Science-Physics in 2013 from the National University of Colombia. In the same year, he started his studies in Solid State Physics at Uppsala University, Sweden, and received his Lic. Phil. degree in 2015 and his PhD in 2017. Currently, he is a postdoctoral researcher at the Department of Chemistry, University of Zurich. His research interests focus on the implementation of computational methods, theoretical and experimental approaches to understand the structure, transport, magnetic, optical, electronic properties and application of solidstate nanostructures, metal oxides, thin films and hybrid organicinorganic systems. 


\section{The quest for reliable water oxidation catalysts (WOCs)}

Finding efficient ways to capture and store renewable solar energy in chemical bonds through artificial photosynthesis (AP), e.g. water splitting, is currently one of the most competitive and intensely researched topics. ${ }^{1,2}$ To date, the large-scale production of renewable hydrogen via AP is significantly constrained by the lack of efficient, low cost and durable WOCs. ${ }^{3,4}$ This is due to the many unresolved fundamental issues of the energetically and kinetically demanding four-electron transfer oxygen evolution reaction (OER):

$$
2 \mathrm{H}_{2} \mathrm{O} \rightarrow 4 \mathrm{H}^{+}+4 \mathrm{e}^{-}+\mathrm{O}_{2} \quad E^{0}=1.23 \mathrm{~V} \text { vs. NHE }
$$

Understanding the catalytic mechanisms associated with this multi-electron process is inherently difficult and demands joint

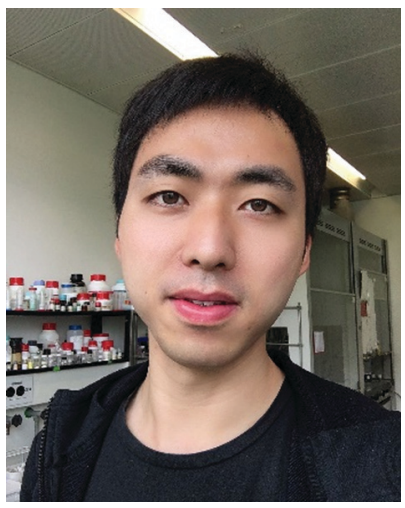

W. Wan
Wenchao Wan received his MSc in Materials Science and Engineering from Southwest Petroleum University in 2016. Then he joined Prof. Greta R. Patzke's group to pursue his PhD degree at the University of Zurich. His current research focuses on the design, synthesis and characterization of new types of graphene-based catalysts for energy storage and conversion with special emphasis on singleatom catalysts for electrocatalytic applications.

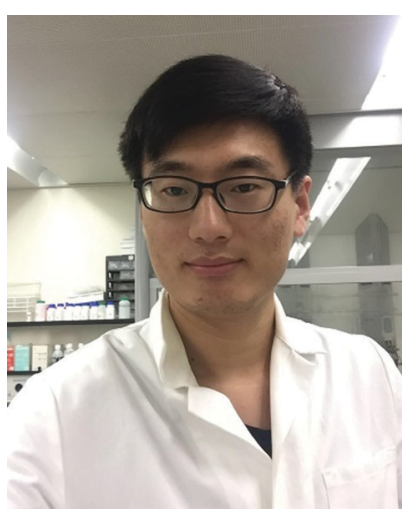

Y. Zhao
Yonggui Zhao received his BSc in Electrical Engineering from Northeastern University (2014) and his MSc in Solid-state Electronics from Tianjin University (2017). After working as a Junior Researcher in experimental physics at the University of Amsterdam, he joined the group of Prof. Greta R. Patzke as a PhD candidate in University of Zurich (December 2017). He is currently working on nanostructured materials in electrocatalysis.

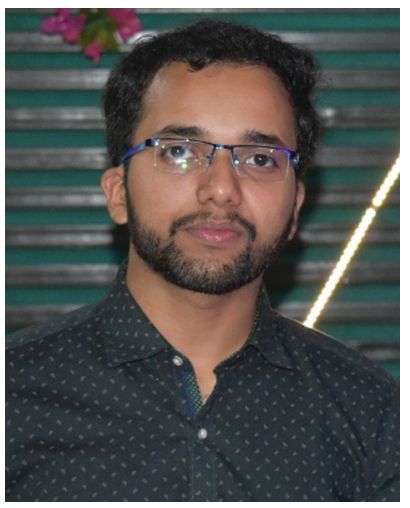

D. P. Adiyeri Saseendran oxidation catalysts using combined synchrotron X-ray and conventional spectroscopic techniques, together with their immobilization on metal oxide surfaces for photo/electrocatalytic water oxidation.

efforts from multidisciplinary collaborations. ${ }^{5}$ Over the past decades, numerous WOCs have been designed and evaluated, ranging from small molecular nuclearities to heterogeneous materials. ${ }^{6,7}$ A remarkable arsenal of optimization strategies and mechanistic models have been accumulated for both molecular and heterogeneous WOCs. ${ }^{1,8}$ Herein, we employ the term 'molecular' instead of 'homogeneous' because not all molecular WOCs operate in a homogeneous solution, such as immobilized molecular WOCs and molecular assembles. Meanwhile, the term 'heterogeneous' WOCs here is also extended beyond the conventional view that the catalytic activity arises from surface sites only, for example, the internal metal sites of a crystalline solid were shown to be active when amorphization occurs. $^{9}$ Therefore, an evolutionary concept of molecular and heterogeneous WOCs is required to integrate new, overarching insights into catalytic processes. ${ }^{10}$ Forefront research increasingly

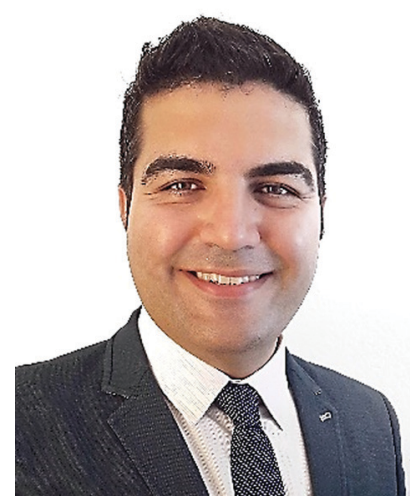

S. E. Balaghi
S. Esmael Balaghi received his BSc in Chemistry from Shiraz University and MSc in Inorganic Chemistry from the Institute for Advanced Studies in Basic Sciences (IASBS). In 2016, he joined Prof. Greta R. Patzke's research group as a PhD candidate at the University of Zurich. He is currently working on in situ electrochemical studies of water splitting catalysts with different operando techniques such as X-ray absorption spectroscopy and in situ electrochemical liquid-cell electron microscopy methods. 
aims to move beyond the conventional differentiations between molecular and heterogeneous design concepts and explore the mutual exchange between both communities. However, clear identification and isolation of molecular compounds that truly bridge both fields remain challenging. ${ }^{11,12}$

Further, limiting factors in both molecular and heterogeneous WOC research include the lack of operational durability on the molecular side and insufficient molecular-level understanding of heterogeneous catalysts on the other side. ${ }^{13}$ Thus, to promote progress on both fronts, innovative ways to solve these inherent problems are required, together with new strategies for reciprocal knowledge transfer. A common starting point is the involvement of equivalent reactive moieties in both molecular and heterogeneous WOCs at the atomic level. ${ }^{14}$ Specifically, the surface of a finite particle offers localized molecular centers for catalytic reactions, while some key molecular motifs are essentially surface cut-outs of solids. These structural similarities clearly link both fields for a mutual transfer of insight, which is beneficial and indispensable for optimizing the performance and durability of WOCs in general.

In this review, we discuss new strategies for knowledge transfer between both molecular and heterogeneous WOC research after a survey of their recent individual research progress (with main focus on first-row transition metal (TM)-based systems of application-oriented and fundamental interest). In Part I, the advantages of molecular WOCs are highlighted in terms of their nuclearity, mechanistic understanding and clear structure-activity correlations. This enables the targeted design of molecular WOCs with desired functionality, such as mimics of Nature's photosystem II (PSII). The spectrum of current optimization strategies such as engineering the metal nuclearity, tuning the electronic properties of the surrounding ligands, and introducing intramolecular proton regulation sites is concisely summarized. In addition, the importance of molecular integrity is addressed. Part II starts with advances in the mechanistic exploration of heterogeneous
WOCs, taking Co- and Fe-oxides as representative and deeply explored examples of transition metal-based WOCs. We highlight the dynamic reaction pathways that are associated with material properties and experimental conditions. Further, emerging heterogeneous optimization strategies such as substitution of secondary elements, vacancies, lattice strain and crystal planes are discussed. Additionally, we also acknowledge the controversial topics of surface amorphization and real active centers of some classic heterogeneous WOCs. Finally, in Part III, the trends in the mutual knowledge transfer between both sides are reviewed. We begin with exploring the optimization of heterogeneous WOCs as tools for the molecular realm, such as the translation of reactive site motifs and application of the scaling relations. Furthermore, several molecular strategies emerging from heterogenized single-atom catalyst systems as models are introduced, such as structural features of the support and catalyst, coordination engineering and cooperative metal centers. Finally, emerging joint molecular and heterogeneous systems are discussed to highlight the advantages of combining both fields directly.

\section{Part I: recent developments and mechanistic studies of molecular WOCs}

\subsection{Molecular WOCs as platforms for accessing reaction mechanisms}

1.1.1. Atomic-scale access to active sites. A precise understanding of the molecular structure at increasing atomic resolution is an essential step toward the rational design of efficient molecular WOCs. Over the last years, a number of studies have tackled this issue using the diffraction, scattering, microscopy and spectroscopy techniques. ${ }^{15,16}$ To some extent, this has solved many challenging molecular structures and allowed their active sites to be characterized in detail. However, cutting-edge experiments using high energy radiation sources

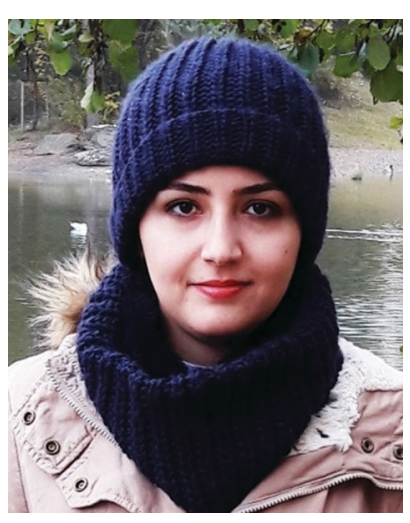

S. Heidari
Sima Heidari is a Postdoctoral Researcher at the University of Zurich in the group of Prof. Greta $R$. Patzke. She received her MSc from the Institute for Advanced Studies in Basic Sciences (IASBS) in 2012, and her $P h D$ degree from the University of Tabriz in 2017. Her current research is focused on developing earth-abundant transition metal-based electrocatalysts for the water oxidation reaction.

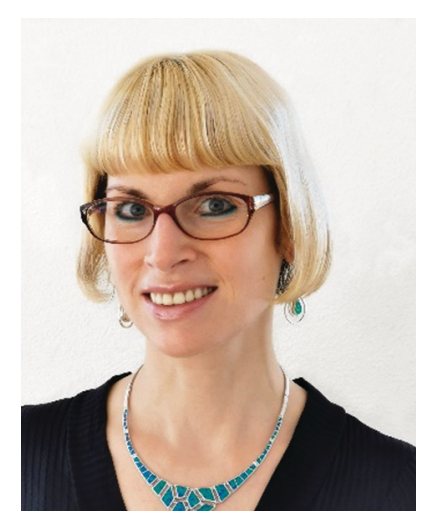

G. R. Patzke

Greta $R$. Patzke received her PhD from the University of Hannover and her venia legendi at ETH Zurich. She then moved to the University of Zurich, where she was promoted to Full Professor in 2016. Her research interests include the hydrothermal synthesis and monitoring of nanomaterials for environmental applications, together with the catalytic properties and bio-medical applications of polyoxometalates. She is a Board Member of the UZH Research Priority Program "Light to Chemical Energy Conversion", where her group investigates a wide range of molecular, nanostructured and solidstate transition metal-based catalysts for water oxidation. 

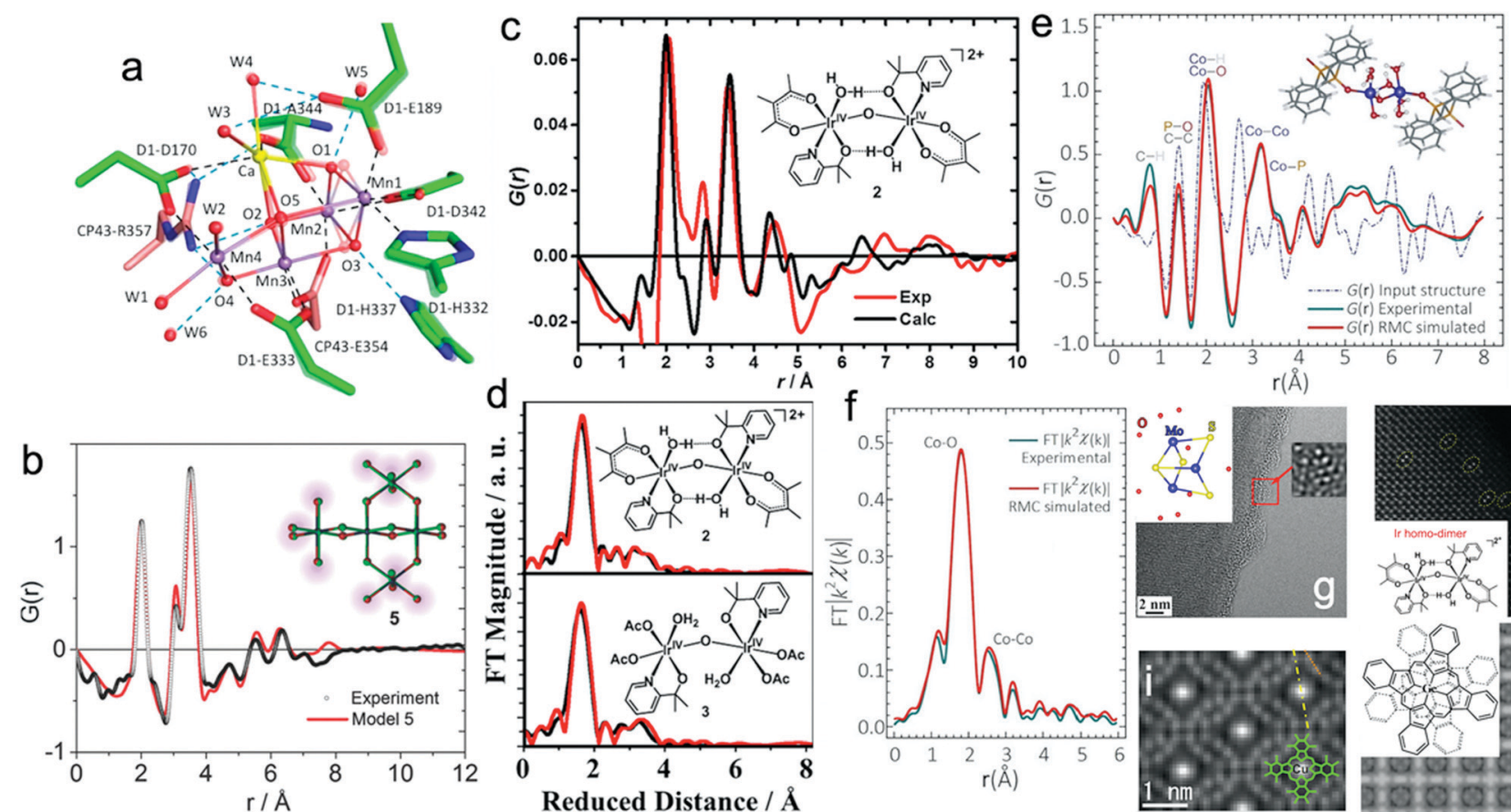

Fig. 1 (a) Superposition of two different structures around the $\mathrm{Mn}_{4} \mathrm{CaO}_{5}$ cluster derived from low-dose XRD-XFEL experiments. Reproduced with permission from ref. 19. Copyright 2017 The American Chemical Society. (b) High-energy G(r) function (PDF) for the structure of an amorphous Ir-based WOC. Reproduced with permission from ref. 20. Copyright 2014 The Royal Society of Chemistry. (c) and (d) Experimental and calculated G(r) function and EXAFS spectra of a C ${ }^{*} \mid r^{\prime \prime I}$ WOC for chemical and electrochemical activation. Reproduced with permission from ref. 21. Copyright 2016 The American Chemical Society. (e) and (f) Experimental and calculated $G(r)$ function and EXAFS spectra of Co-dppeO ${ }_{2}$. Reproduced with permission from ref. 22. Copyright 2019 Elsevier. (g) Low dose-rate HRTEM image for the incomplete cubane-cluster $\left[\mathrm{Mo}_{3} \mathrm{~S}_{4}\left(\mathrm{H}_{2} \mathrm{O}\right)_{9}\right] \mathrm{Cl}_{4}$. Reproduced with permission from ref. 32. Copyright 2020 Elsevier. (h) HAADF-STEM image of an immobilized molecular dinuclear Ir WOC on an $\alpha$ - $\mathrm{Fe}_{2} \mathrm{O}_{3}$ support. Reproduced with permission from ref. 31. Copyright 2018 National Academy of Sciences. (i) LAADF-STEM image of $\mathrm{Cl}_{16}$-CuPc. Reproduced with permission from ref. 29. Copyright 2008 Elsevier. (j) LAADF-STEM image of (GeOPc) $n$. Reproduced with permission from ref. 30. Copyright 2008 Taylor \& Francis.

are required for truly accessing the molecular structure and the active moieties of sensitive catalysts at maximum atomic-level resolution when conventional X-ray crystallography is not an option. ${ }^{17}$ Considering that many molecular WOCs suffer from structural "radiation-damage" at high-dose energy experiments, advanced methods based on radiation damage-free structural characterizations have attracted significant interest and considerable developments. ${ }^{18}$

Recently, X-ray-free electron laser (XFEL) diffraction was used to solve the structure of metastable intermediates in the $\mathrm{Mn}_{4} \mathrm{CaO}_{5}$ complex of Nature's PSII at increased atomic resolutions of 1.85-1.87 $\AA^{19}$ These low-dose XFEL experiments showed the presence of two environments around two monomers with different hydrogen bonding in the dimeric $\mathrm{Mn}_{4} \mathrm{CaO}_{5}$ moiety (Fig. 1a). In another representative study, the high-energy $\mathrm{X}$-ray pair distribution function (PDF) was used to resolve the structure of an amorphous iridium-oxide WOC. The authors suggested that the peak at $3.07 \AA$ in the PDF (Fig. 1b) ${ }^{20}$ resembles the local structures found in $\mathrm{Cp}^{*} \operatorname{Ir}(\mu-\mathrm{O})_{3} \operatorname{IrCp}^{*}, \operatorname{Ir}_{2} \mathrm{Cl}_{2}(1,5$-cyclooctadiene $)_{2}(\mu-\mathrm{OH})_{2}(\mu-\mathrm{O})$, and alkoxy derivatives featuring non-planar $(\mu-\mathrm{O}(\mathrm{R}))_{2}$ geometry. According to these results, the authors proposed that the local structure of amorphous Ir-oxide WOCs comprises distorted $\operatorname{Ir}(\mu-\mathrm{O})_{3} \operatorname{Ir} / \operatorname{Ir}(\mu-\mathrm{O})_{2} \operatorname{Ir}$ substructures. A similar study on active $\mathrm{Cp}^{*} \mathrm{Ir}^{\mathrm{III}}$ complexes $\left(\mathrm{Cp}^{*}=\text { pentamethyl-cyclopentadienyl }\right)^{20,21}$ showed that the local structure of these complexes features $\operatorname{~Ir~}^{\mathrm{NV}}$-dimers of mono- $\mu-\mathrm{O}$ cores with terminal anionic ligands (Fig. 1c). ${ }^{21}$ Recently, some of us applied reverse Monte Carlo (RMC) modeling of PDF data to unravel the atomic range structure of a disordered $1 \mathrm{D}$ cobalt coordination polymer catalyst $\left(\mathrm{Co}-\mathrm{dppeO}_{2}\right)^{22}$ Our results revealed that the structure of $\mathrm{Co}^{-} \mathrm{dppeO}_{2}$ consists of hydrated edge-site $\left\{\mathrm{H}_{2} \mathrm{O}-\right.$ $\left.\mathrm{Co}_{2}(\mathrm{OH})_{2}-\mathrm{OH}_{2}\right\}$ motifs, nested within an organic matrix of bridging hydrophobic $\mathrm{dppeO}_{2}$ ligands (Fig. 1e).

Synchrotron X-ray absorption spectroscopy (XAS) also provides access to the electronic structure and coordination environment of active sites in molecular WOCs. ${ }^{23}$ XAS has been broadly used to study the electronic and coordination structure of metal centers, such as in $\left\{\mathrm{CaMn}_{4} \mathrm{O}_{5}\right\},{ }^{24} \mathrm{Cp}^{*} \mathrm{Ir}^{\mathrm{III}},{ }^{21}$ $\left[\mathrm{Cp} * \mathrm{Ir}(\right.$ pyalc)Cl $],{ }^{25}\left\{\mathrm{Co}_{4} \mathrm{O}_{4}\right\} /\left\{\mathrm{Co}_{x} \mathrm{Ni}_{4-x} \mathrm{O}_{4}\right\}$ cubanes, ${ }^{26}$ Co-dppeO ${ }_{2},{ }^{22}$ polyoxometalates (POMs) ${ }^{27}$ and Ru-bda (bda; 2,2'-bipyridine-6, $6^{\prime}$ dicarboxylate) ${ }^{28}$ among other molecular WOCs.

Since XAS is sensitive to local orders up to interatomic distances of $\sim 4 \AA$ around the absorbing atom, XAS data can be well complemented with PDF data, which offers structural information up to $12-15 \AA$.

In this case, the structural correlations in the nearest coordination shells around the active metal sites are obtained from XAS data, and the extended structures of molecular ligands are extracted from the PDF data. This allows the domain size and structure of molecular WOCs to be elucidated, which are not accessible for conventional single crystal X-ray 
diffraction, including the first shell, outer sphere and longrange distances that are not accessible from XAS data alone. ${ }^{23}$ Recent studies on molecular $\mathrm{Cp}^{*} \mathrm{Ir}^{\mathrm{III}}$ (Fig. 1c and d) ${ }^{21}$ and our work on the disordered coordination polymer Co-dppeO ${ }_{2}(\mathrm{dppe}=$ 1,2-bis(diphenylphosphino)ethane; Fig. 1e and f) ${ }^{22}$ combine high-energy PDF and XAS data with molecular dynamics (MD), density functional theory (DFT), and RMC modeling to solve the respective molecular structures. These unified methods allow the seamless correlation of experimental data at multiple atomiclength scales. This approach provides more realistic and extended 3D model structures of complex molecular WOCs, which cannot be achieved from conventional crystallographic analyses.

Imaging techniques such as transmission electron microscopy (TEM), scanning transmission electron microscopy (STEM), and scanning tunneling microscopy (STM) offer access to structural investigations with atomic resolution at the subnanometer scale. ${ }^{16}$ This is especially of interest for molecules on supports or electrode surfaces (see Part III). However, the short mean-free path of electrons limits standard TEM methods to ultra-high vacuum (UHV) conditions and the aberration of magnetic lenses limits the application range of STEM/STM imaging. ${ }^{16,23}$ In STEM, a focused convergent electron beam scans a specific sample area to produce signals, which are recorded spot-by-spot for imaging. ${ }^{16,23}$ Depending on the type of detector used to obtain STEM images, they are classified as (i) bright field (BF-STEM), which detects the intensity in the direct beam after passing a point on the sample, (ii) annular dark field (ADF-STEM), which uses scattered/diffracted electrons through small angles to create an image, and (iii) high-angle annular dark field (HAADF-STEM), which detects scattered electrons at high angles arising mainly from incoherent scattering. ${ }^{16,23}$ ADF-STEM is sensitive to the atomic number $Z$, and thus the presence of heavier elements in a specimen increases the intensity of the image. This renders ADF-STEM a useful technique to image metal atoms embedded in a matrix of lower atomic numbers. To gain insight into the elemental composition, the STEM setup is often combined with electron energy loss spectroscopy (EELS) and energy dispersive X-ray spectroscopy (EDS). ${ }^{16,23}$

ADF-STEM was used to obtain Z-contrast images of the organic molecular structure in hexadecachloro-Cu-phthalocyanine $\left(\mathrm{Cl}_{16}\right.$-CuPc), polyhalogenated CuPc-derivatives ( $\left.\mathrm{CuPc}-\mathrm{Br} /-\mathrm{Cl}\right)$ (Fig. 1i) ${ }^{29}$ hexadecachloro-Cu-phthalocyanine $\left(\mathrm{Cl}_{16} \mathrm{CuPc}\right),{ }^{29}$ and poly-Ge-oxy-phthalocyanine $(\mathrm{GeOPc})_{n}$ (Fig. $\left.1 \mathrm{j}\right){ }^{30}$ Recently, HAADF-STEM was used to investigate the immobilization of a molecular dinuclear Ir-based WOC after photochemical removal of organic ligands by bridged $\mathrm{H}_{2} \mathrm{O} / \mathrm{OH}$ groups, which prevents detachment and aggregation (Fig. 1h). ${ }^{31}$

Advances in aberration-corrected STEM (AC-STEM) have further increased the atomic resolution to the sub-Ångström scale. This allows isolated molecules to be directly imaged on supporting layers through proper sample preparation. ${ }^{16}$ In a recent representative work, the molecular structure of a trimeric, incomplete aqua ion cubane-cluster $\left(\left[\mathrm{Mo}_{3} \mathrm{~S}_{4}\left(\mathrm{H}_{2} \mathrm{O}\right)_{9}\right] \mathrm{Cl}_{4}\right)$ was directly derived from AC-STEM imaging (Fig. 1g). ${ }^{32}$ The results showed that e-beam-sample interaction is a key factor to obtain one-to-one correspondence between the acquired images and atomic structure. Moreover, low-dose techniques must be further developed for increasing the resolution of the molecular structure.

1.1.2. Establishing structure-function relations for molecular WOCs. Excellent cases of studies on catalytic performance driven by structure-function relations are those focused on tuning the nature of organic ligands. The stability/reactivity of high-valent metal-oxo species created during proton-coupled electron transfer (PCET) are largely controlled by the structure of the coordinating ligand. Consequently, ligand tuning can strongly enhance or eventually negatively impact the catalytic functions. As a design principle for molecular WOCs, the organic ligand must be structurally stable and oxidatively robust against harsh reaction conditions in aqueous solution. Furthermore, the structural topology and geometry of the ligand must be optimized to tune the stability and reactivity of the high-valent metal-oxo intermediate species. ${ }^{33}$ The coordination number and short-range structure of the selected organic ligands around the active metal sites must promote conjugation effects, charge transfer, $\pi$-accepting and $\sigma / \pi$ donating capabilities, hydrogen bonding, steric hindrance and hydrophilicity. Molecular Ru-bda WOCs (bda = 2,2'-bipyridine-6, $6^{\prime}$-dicarboxylate), ${ }^{32}$ for example, it has been shown that applying a negatively charged carboxylate group for ligand design is an effective strategy to lower their onset potentials, stabilize high-valent intermediates and facilitate the proton transfer in molecular WOCs. The structural distortion of a metal complex by ligand design creates open catalytic sites for binding the substrate as an additional ligand, which offers a novel concept for designing highly efficient molecular WOCs. The influence of negatively charged $\mathrm{H}_{3} \mathrm{pbc}^{3-}$ ligands $\left(\mathrm{H}_{3} \mathrm{hpbc}=\right.$ 2(2-hydroxyphenyl)-1 $H$-benzo[d]-imidazole-7-carboxylic acid) on the activity of a $\mathrm{Ru}^{\mathrm{III}}$ complex (Fig. $\left.2 \mathrm{~b}\right)^{34}$ showed that $\mathrm{O}-\mathrm{O}$ bond formation proceeds though the $\mathrm{Ru}^{\mathrm{VI}}$ species, whose accessibility is driven by non-innocent organic ligands. The distortion of metal complexes and negatively charged ligands can be efficiently achieved through ligand design, and this strategy can be applied in the construction of high-performance earthabundant metal-based molecular WOCs. Accordingly, Yan et al. ${ }^{35}$ studied the effect of ligand tuning on the catalytic activity of a series of $\mathrm{Mn}_{12} \mathrm{O}_{12}(\mathrm{OAc})_{16-x} \mathrm{~L}_{x}\left(\mathrm{H}_{2} \mathrm{O}\right)_{4}$ molecular WOCs ( $\mathrm{L}=$ acetate, benzoate, benzenesulfonate, diphenylphosphonate and dichloroacetate). Although the highest activity was achieved for a set of mixed acetate-phosphonate ligands, two distinct structurefunction relationships control the catalytic behavior (Fig. 2a). Firstly, complexes undergoing at least one-electron oxidation display activity ( $\mathrm{pH} 7 ; 640-820 \mathrm{mV} ; 0.2 \mathrm{~mA} \mathrm{~cm}^{-2}$ ), while failing to be oxidized renders the complex inactive. Secondly, sitedisorder at the active $\mathrm{Mn}_{1}$ (cubane core) and $\mathrm{Mn}_{3}$ (aqua-ligated) centers lead to higher catalytic activity.

Tuning the local structure of metal complexes by leaving coordination sites labile or open to act as active catalytic centers is another strategy towards highly active molecular WOCs. In octacobalt-cluster-MOFs, $\left[\mathrm{Co}_{8}(-\mathrm{OH})_{6}(\mathrm{bdt})_{4}(\mathrm{Hbdt})_{2}\right]$, featuring a network of linked $\mathrm{Co}_{8}\left(\mathrm{~m}_{4}-\mathrm{OH}\right)_{6}(\mathrm{Rtrz})_{12}(\mathrm{Rtrz}=1,2,3-$ triazolate) clusters and $\mathrm{bdt}^{2-}$ ligands $\left(\mathrm{bdt}^{2-}=1,2\right.$-benzenedithiolate), it was shown that at each face of $\left\{\mathrm{Co}_{8}\left(\mu_{4}-\mathrm{OH}\right)_{6}\right\}$, the 

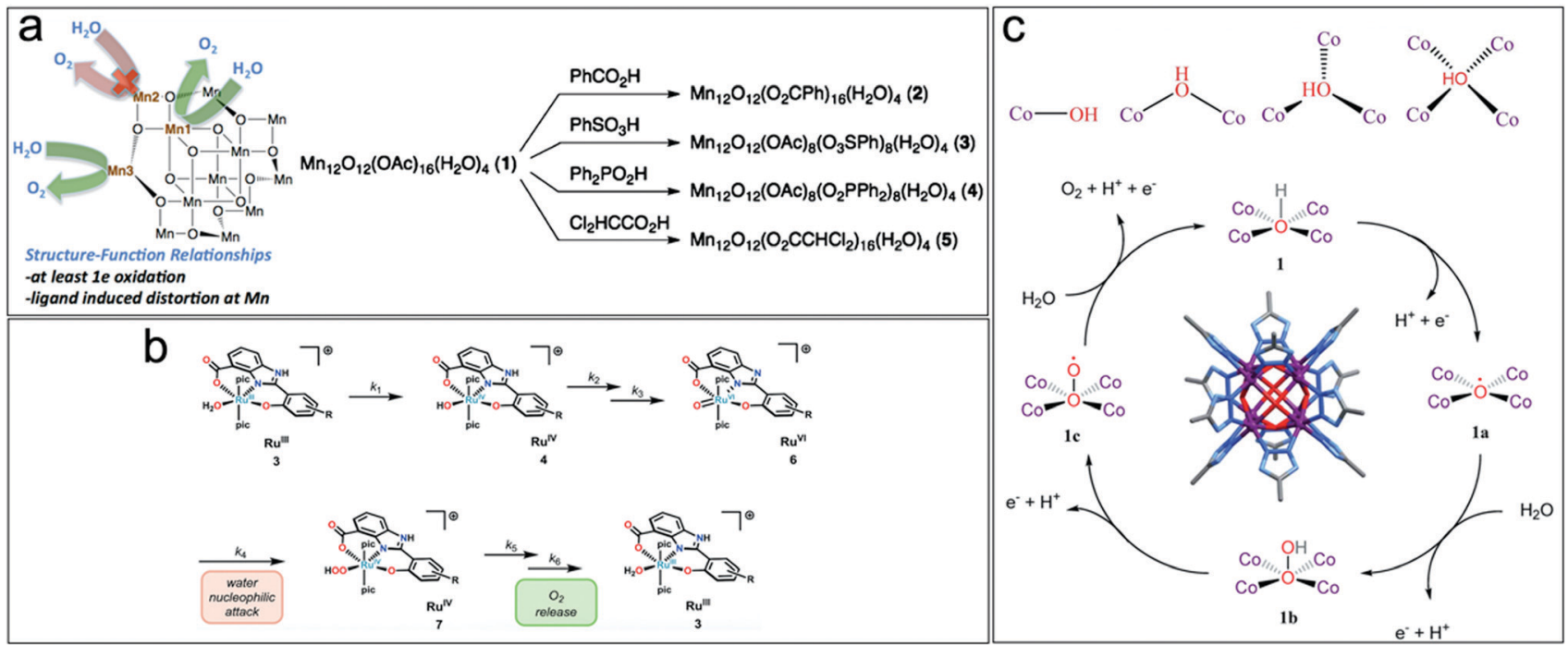

Fig. 2 (a) Structure-function relationship and ligand exchange synthesis of $\mathrm{Mn}_{12} \mathrm{O}_{12}(\mathrm{OAc})_{16-x} \mathrm{~L}_{x}\left(\mathrm{H}_{2} \mathrm{O}\right)_{4}$ WOC. Reproduced with permission from ref. 35 . Copyright 2015 The American Chemical Society. (b) Steps for the release of $\mathrm{O}_{2}$ from Ru-aqua complexes on Rull' complexes containing negatively charged $\mathrm{hpbc}^{3-}$ ligands. Reproduced with permission from ref. 34. Copyright 2016 The Royal Society of Chemistry. (c) Reactant hydroxyl ion coordinated to cobalt ions during the OER, $\mathrm{Co}_{8}\left(\mathrm{~m}_{4}-\mathrm{OH}\right)_{6}(\mathrm{Rtrz})_{12}$ cluster and reaction mechanism for $\left[\mathrm{Co}_{8}(-\mathrm{OH})_{6}(\mathrm{bdt})_{4}(\mathrm{Hbdt})_{2}\right]$ WOC for photodriven water oxidation. Reproduced with permission from ref. 36. Copyright 2019 The Royal Society of Chemistry.

hydroxyl anion is linked to four coplanar $\mathrm{Co}^{\mathrm{II}}$ centers in a $\mu_{4}$-coordination to form a $\left\{\mathrm{Co}_{4}\left(\mu_{4}-\mathrm{OH}\right)\right\}$ unit. ${ }^{36}$ The $\mu_{4}$-OH group contributes to the activity by delivering an oxygen vacancy site with the optimal $\mathrm{OH}^{-}$adsorption energy. The $\mathrm{OH}^{-}$species are stabilized by four coplanar Co ions, becoming more stable with an increase in oxygen coordination, which further enhances the catalytic performance (Fig. 2c). ${ }^{36}$ Multi-nuclear molecular structures are often well suited for facilitated multi-electron transfer, i.e. highly active molecular WOCs. ${ }^{37}$ However, for this WOC design, it must be considered that the number of d-electrons of the transition metal drives the stability/reactivity of the resulting metal-oxo species. Transition metals with a moderate number of d-electrons $\left(\mathrm{Mn}^{\mathrm{V}}-\mathrm{d}^{2}, \mathrm{Fe}^{\mathrm{V}}-\mathrm{d}^{3}, \mathrm{Ru}^{\mathrm{V}}-\mathrm{d}^{3}, \mathrm{Fe}^{\mathrm{IV}}-\mathrm{d}^{4}\right)$ efficiently produce reactive oxo species since they offer suitable empty d-orbitals to accept the $2_{\mathrm{p} x} / 2_{\mathrm{p} y}$ electrons from the oxo ligand. Metals with low numbers of d-electrons $\left(\mathrm{V}^{\mathrm{V}}-\mathrm{d}^{0}, \mathrm{Ti}^{\mathrm{iV}}-\mathrm{d}^{0}\right.$, and $\mathrm{Zr}^{\mathrm{IV}}-\mathrm{d}^{0}$ ) are inactive in $\mathrm{O}-\mathrm{O}$ bond formation. Filling of the $\mathrm{e}\left(\mathrm{d}_{x z} ; \mathrm{d}_{y z}\right)$ orbitals in $\mathrm{Co}, \mathrm{Ni}$, and $\mathrm{Cu}$ furthermore renders the formation of multiple metal-oxo species difficult. ${ }^{33}$

Overall, these trends pave the way for the development of highly efficient molecular WOCs by exploiting the wide combinatorial space of various metal atoms with negatively charged ligands (pyrrole, $\mathrm{hpbc}^{3-}$, carboxylate, sulfonic and phenolic groups) to efficiently regulate the stability/reactivity of highvalent metal-oxo species, which are considered key intermediates in water oxidation catalysis.

\subsection{Recent progress in designing molecular WOCs}

In this part, our discussion will primarily focus on recent progress on first-row TM-based ( $\mathrm{Fe}, \mathrm{Co}, \mathrm{Ni}$ and $\mathrm{Cu}$ ) molecular WOCs. For the widely studied Ru and Ir-based molecular WOCs, we refer to some excellent topical reviews. ${ }^{8,33,38,39}$ Here, instead of comparing their catalytic activity, we summarize their mechanistic aspects, namely (i) experimental trapping of high-valent metal centers, (ii) reaction mechanisms for $\mathrm{O}-\mathrm{O}$ bond formation and the involvement of non-innocent ligands, (iii) electronic and structural tuning of ligand substitutions, and (iv) non-negligible role of reaction electrolytes.

1.2.1. Mononuclear WOCs. The mononuclear WOCs referred to in the following are structurally represented in Fig. 3. First, to accumulate sufficient oxidative energy, the involvement of high-valence metal sites is widely proposed for the first-row TMs and some of them (such as for Fe and Co) have been verified experimentally. Various spectroscopic probing techniques were successfully implemented for this purpose. Among them, ultraviolet-visible (UV-vis) spectroscopy is the most accessible and powerful approach. $\mathrm{Fe}^{\mathrm{IV}}$-oxo species, for example, display a characteristic broad band absorption between 700 and $800 \mathrm{~nm}$ in their UV-vis spectra, ${ }^{40-42}$ and the band positions of the maximum absorption are slightly shifted with structural variations in the Fe-based mononuclear WOCs 1-4. ${ }^{43-46}$ In the case of compound 3 , its high spin paramagnetic intermediate also exhibits characteristic electron paramagnetic resonance (EPR) signals upon immediate quenching of the catalytic reaction. The oxidation state of the intermediate species was traced by its Fe K-edge XAS signature. ${ }^{45}$ Moreover, after separation from the reaction mixture via high performance liquid chromatography (HPLC), the molecular weight of the intermediate for complex $\mathbf{1}$ was determined by mass spectroscopy (MS), which further corroborated the above characterizations. ${ }^{43}$ Some groups even experimentally trapped $\mathrm{Fe}^{\mathrm{V}}$-oxo species for mononuclear WOC 5 with lab-scale techniques such as UV-vis and EPR, and complementary techniques, e.g. Mössbauer spectroscopy, were used to confirm this unusual 


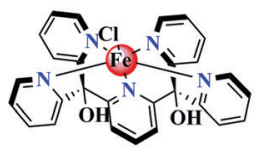

1

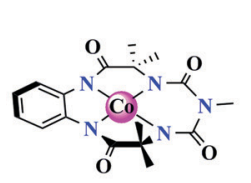

7

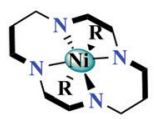

13

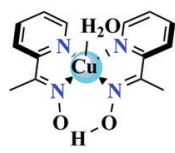

19

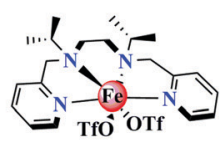

2

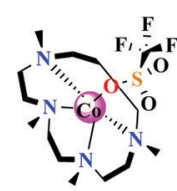

8

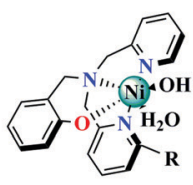

14

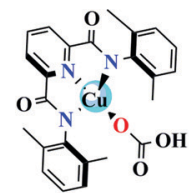

20

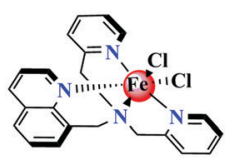

3

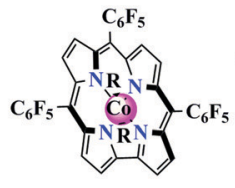

9

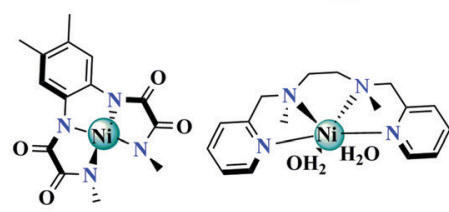

16

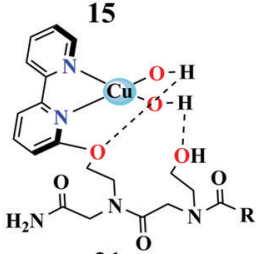

21

4

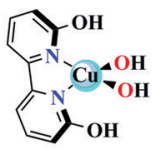

22
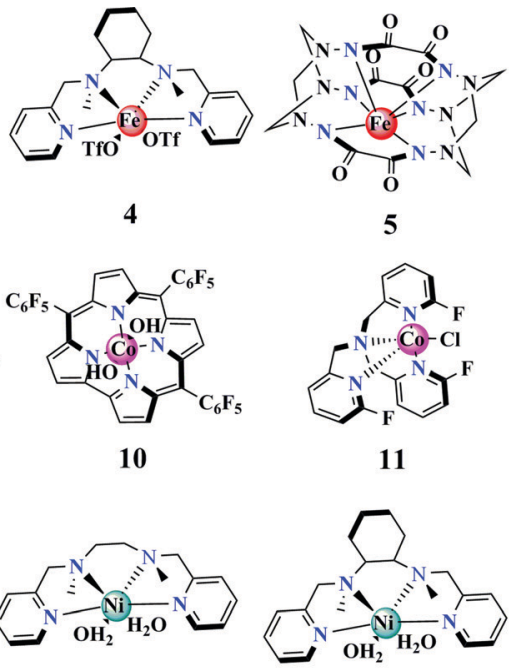

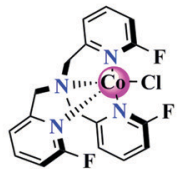

11

17

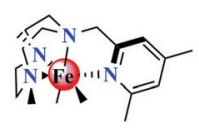

6

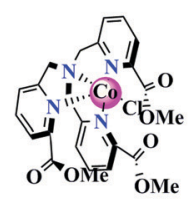

12

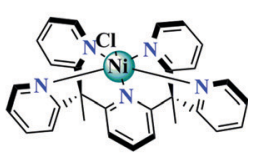

18

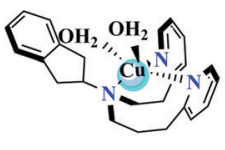

23

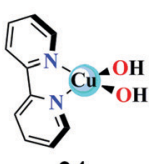

24

Fig. 3 Molecular structures of the recently developed TM-based mononuclear WOCs discussed in Section 1.2.1.

reaction intermediate. ${ }^{47}$ It is interesting that these metastable and highly oxidizing species feature distinct lifetimes depending predominantly on the oxidation state of their metal center. For instance, the $\mathrm{Fe}^{\mathrm{IV}}$-oxo species can persist up to hours, as indicated by the slow decay of its absorbance band, ${ }^{43}$ while the $\mathrm{Fe}^{\mathrm{V}}$-oxo species only survives for minutes or even shorter timescales. ${ }^{47}$ Furthermore, the molecular design and measurement conditions also play significant roles in the measured lifetime of these intermediate species. For the Co-based mononuclear WOCs 7-10, only $\mathrm{Co}^{\mathrm{IV}}$-oxo intermediate species were trapped experimentally with the help of UV-vis, ${ }^{48-51} \mathrm{EPR},{ }^{50-52}$ Raman and XAS spectroscopy. ${ }^{50}$ No spectroscopic signature of $\mathrm{Co}^{\mathrm{V}}$-oxo intermediate species was found, although some indications had been derived from the cyclic voltammetry (CV) measurements. ${ }^{48,52}$ In the case of $\mathrm{Ni}$ - and $\mathrm{Cu}$-based mononuclear WOCs, there was no clearly resolved spectroscopic signal available for either the $\mathrm{Ni}^{\mathrm{IV}}$ or $\mathrm{Cu}^{\mathrm{IV}}$-oxo intermediate species, respectively. ${ }^{1,53,54}$

Second, we notice that the reaction mechanism of these first-row TM-based mononuclear WOCs is predominantly proposed as water nucleophilic attack (WNA) to the above discussed high-valent metal-oxo species or metal-oxyl radicals, where $\mathrm{O}-\mathrm{O}$ bonds are formed..$^{52,55-57}$ In this case, first-order reaction kinetics is normally observed for the mononuclear WOCs, ${ }^{58}$ which means that the rate-determining step (RDS) involves only one metal center. Meanwhile, intra- (via the formation of binuclear WOCs) and inter-molecular oxo coupling (I2M) pathways for $\mathrm{O}-\mathrm{O}$ bond formation are also proposed as alternative reaction mechanisms. ${ }^{43,59,60}$ These reaction pathways are particularly validated for Fe- and Co-based mononuclear
WOCs, where high-valent intermediate species were experimentally trapped. ${ }^{61}$ However, for $\mathrm{Ni}$ - and $\mathrm{Cu}$-based systems, the validation of these classic mechanisms faces significant challenges since the existence of high-valent species is under debate. ${ }^{62-64}$ Instead, reaction mechanisms involving noninnocent ligands are widely proposed in this regard. ${ }^{65,66}$ For instance, the carbonate and phenolate ligands in Ni-based mononuclear WOCs $\mathbf{1 3}$ and $\mathbf{1 4}$ were believed to be redoxactive centers in addition to the metal sites. ${ }^{67,68}$ Accordingly, high-valent metal centers can be circumvented for charge accumulation. The theory of non-innocent ligands was also employed for the Cu-based mononuclear WOC 19, where the redox active aryl oxime ligand was used to store oxidizing equivalents. ${ }^{69}$ Another important feature of these noninnocent ligands is that they can act as intramolecular bases to facilitate the proton removal for water activation, for example the carbonate and hydroxyl groups in Cu-based mononuclear WOCs 20-22. ${ }^{65,70,71}$ Therefore, the cooperative effect between the proton acceptor ligand and the catalytic metal center can help to minimize the energy barrier for $\mathrm{O}-\mathrm{O}$ bond formation.

Here, it should be noted that the detailed reaction mechanism is not a stationary concept. On the contrary, it can be dynamic depending on the reaction conditions. For example, two labile ligands in the cis configuration were involved for Fe-based mononuclear WOC 1 when water oxidation proceeded in acidic conditions. However, in near-neutral electrolyte, a single labile site was sufficient for the reaction to occur. ${ }^{43}$ Another example is the Cu-based WOC 23, where reversible 
reaction pathways between mono and bi-nuclear (antiferromagnetically coupled) species were identified as a function of electrolyte $\mathrm{pH}^{72}$

Third, many recent studies exhibited significant molecular activity and integrity changes upon a small variation in the ligand substitutions. ${ }^{73}$ We observed that it is hard to obtain unified observations for the ligand substitutions. For example, for the Fe- and Co-based mononuclear WOCs 6, 11, and 12, it was concluded that substituents with higher electronegativity favored higher catalytic activity. ${ }^{74-76}$ Instead, this positive promotion was supported with electron-donating ligands for Ni-based mononuclear WOC $15,{ }^{77}$ which is consistent with the observations for the axial ligand $\mathrm{R}$ ( $\mathrm{R}$ designates pyridine and its derivatives) on WOC 9. ${ }^{52}$ Nevertheless, it has been widely proposed that the activity promotion originates from a lower energy barrier for $\mathrm{O}-\mathrm{O}$ bond formation. In addition, the steric hindrance of the substituents is another option to fine-tune the catalytic activity, while maintaining the required molecular stability. ${ }^{78}$ Ligand engineering still holds the key for the development of efficient and robust mononuclear WOCs. Finally, the nature of the electrolyte has also been proposed to play an important role during the catalytic reaction (complex 24). In another example, the presence of acetate anions could efficiently scavenge protons during the RDS of $\mathrm{O}-\mathrm{O}$ bond formation for the mononuclear Ni-WOC $16 .{ }^{59}$ Similarly, phosphate anions were also explored as proton acceptors for mononuclear WOCs 17 and $18 .{ }^{62,79}$

Overall, while mononuclear WOCs are widely perceived as straightforward models for mechanistic studies, the notable complexity of these single-metal-site systems is often underestimated. Comprehensive studies that monitor not only the metal centers, but also the protecting ligands and the employed electrolytes are essential for their complete understanding. This insight into mononuclear systems is also important for designing higher nuclear first-row TM-based WOCs and singleatom catalysts (SACs), as discussed in the following parts.

1.2.2. Binuclear WOCs. Binuclear WOCs represent attractive alternatives to their mononuclear counterparts by providing a two-site platform, thereby triggering small molecule activation processes that are otherwise inaccessible by single-site systems. Since the development of the first molecular water oxidation catalyst, the so called $\mathrm{Ru}$ "blue dimer", many investigations have confirmed that binuclear metal complexes serve as an excellent choice for the development of WOCs owing to their synergic effects and cooperative mechanisms, which drive complex chemical transformations. ${ }^{80-83}$ The strategic design of binuclear catalysts also benefits from overcoming the major challenges of the activation energy barrier and catalyst instability through charge delocalization, redox balancing and other redox processes between the metal centers, which are comparable and evident in many bio-inorganic and artificial systems. ${ }^{84-87}$ Binuclear WOCs are generally believed to follow the $\mathrm{I} 2 \mathrm{M}$ mechanism, although some exceptions exist. ${ }^{88}$ In binuclear systems, the I2M mechanism is proposed to occur via two possible pathways, depending on the two-site availability and local environment. The majority of mechanisms in binuclear WOCs are believed to proceed via an intramolecular I2M (i-I2M) pathway or the general intermolecular I2M, which is accompanied by the in situ generation of metal dimers via coupling of metal-oxo species. ${ }^{89}$ Initially, mechanistic studies of the well-characterized Ru-Hbpp (bpp ${ }^{-}$: bis(2-pyridyl)3,5-pyrazolate) dimer revealed that $\mathrm{O}_{2}$ evolution occurs through the intramolecular i-I2M pathway, ${ }^{90-92}$ which was later confirmed with its cobalt analogue, revealing the involvement of an initial metal-metal dioxo and a later peroxo species formed as a result of two PCET processes. ${ }^{37,93-95}$

Aiming towards the development of functional synthetic models for the oxygen evolution complex (OEC), the $\mathrm{O}_{2}$ evolution reaction of an Mn-terpy dimeric complex (terpy: $2,2^{\prime}: 6,2^{\prime \prime}-$ terpyridine) was investigated by Brudvig et al., representing one of the first WOCs based on earth abundant metals. ${ }^{81,94}$ Although the initial studies focused mainly on $\mathrm{Mn}$ and Co, the statistics from the past six to seven years clearly show an enormous increase in the development of $\mathrm{Fe}$ - and Cu-based binuclear WOCs. ${ }^{15,37,95}$

We have summarized some model binuclear WOCs in Fig. 4. In 2011, Åkermark et al. developed Mn-based dimer 25, which catalyzes water oxidation under photocatalytic and chemical oxidative conditions, employing $\left[\mathrm{Ru}(\mathrm{bpy})_{3}\right]\left(\mathrm{PF}_{6}\right)_{2}$ as a photosensitizer and $\left[\mathrm{Ru}(\mathrm{bpy})_{3}\right]\left(\mathrm{PF}_{6}\right)_{3}$ as an oxidant. ${ }^{96}$ Standard isotopic labelling experiments using $\mathrm{H}_{2}{ }^{18} \mathrm{O}$ were performed, which revealed that both oxygen atoms in the evolved $\mathrm{O}_{2}$ were contributed by solvent water. Further investigations on the correlation of the electronic effects in these Mn dimer catalysts towards their activity were carried out by synthetically modifying the catalyst structure and employing DFT-based computational calculations. ${ }^{97}$ Among the library of synthetically modified structures, the representatives with an aliphatic terminal carboxylate chain showed the highest rate of $\mathrm{O}_{2}$ evolution, which was attributed to facilitation of PCET processes by the involvement of probable hydrogen bonding interactions between water, carboxylate and a hydroxy Mn unit. In 2019, the Mn-dimer WOC 26 based on the bipyalkH ligand (bipyalkH: (2-([2,2'-bipyridin]-6-yl)propan-2-ol)) was reported by Brudvig et al., containing an anionic donor site capable of stabilizing high oxidation states on the metal. ${ }^{87}$ The Mn-bipyalkH dimer catalyses water oxidation in the presence of the sacrificial oxidant $\mathrm{KHSO}_{5}$. The possible catalytic pathways and intermediates were suggested based on EPR and spectroelectrochemical (SEC) investigations. In situ EPR spectroscopic analysis of the catalytic mixture (catalyst $+\mathrm{KHSO}_{5}$ ), following a serial freezing-thawing and re-freezing protocol, depicted a drastic change from a weak signal to an intense 16-line EPR spectral pattern, which suggested the probable involvement of an unusually highly oxidized $\operatorname{Mn}^{\mathrm{V}}(\mu-$ $\mathrm{O}_{2} \mathrm{Mn}^{\mathrm{V}}$ intermediate. The stabilization of this high-valent $\mathrm{Mn}$ species was associated with the high donicity of the ligand alkoxide group, which was also found to contribute to the redox levelling of the high-valent species $\mathrm{Mn}(\mathrm{II} / \mathrm{II}), \mathrm{Mn}(\mathrm{III} / \mathrm{IV})$ via successive deprotonations, as observed from SEC investigations. This also revealed that the Mn-dimer can be produced in situ from the corresponding Mn-monomers, and that dimer 26 remains dominant with a minor (around $20 \%)\left[\mathrm{Mn}^{\mathrm{IV}}(\right.$ bipyalkH)(bipyalkH) $]\left(\mathrm{PF}_{6}\right)_{2}$ monomer content above an applied potential of $900 \mathrm{mV} v s$. NHE. 

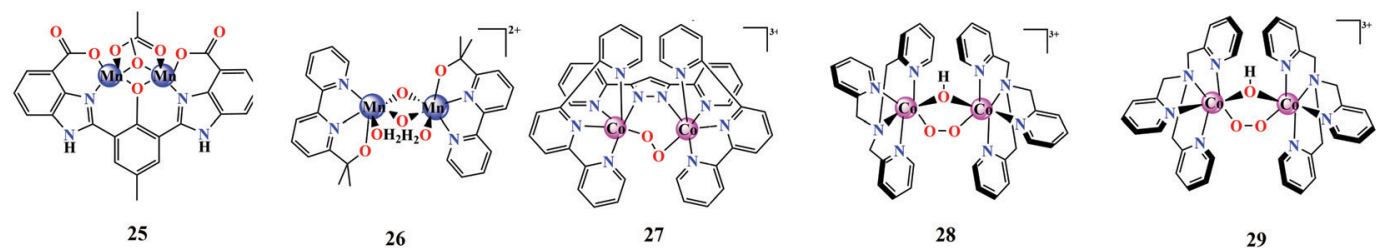

28
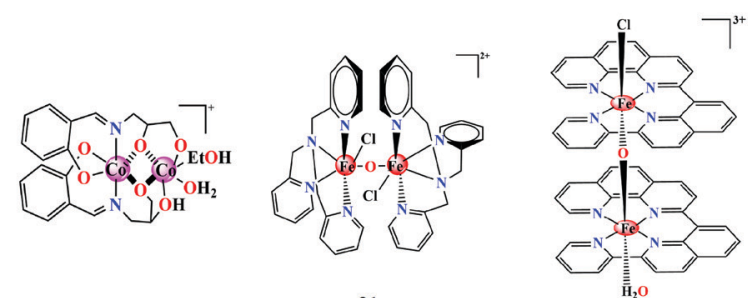

32
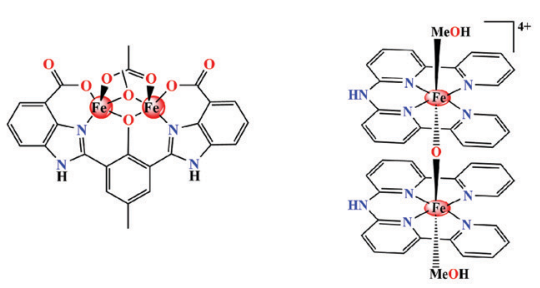

30

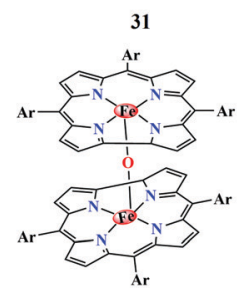

33

34
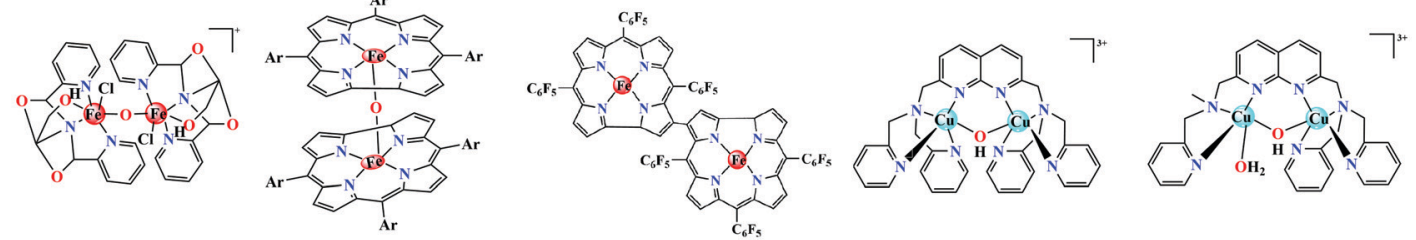

35

36
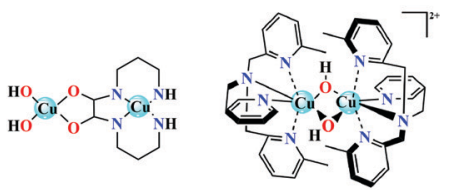

37
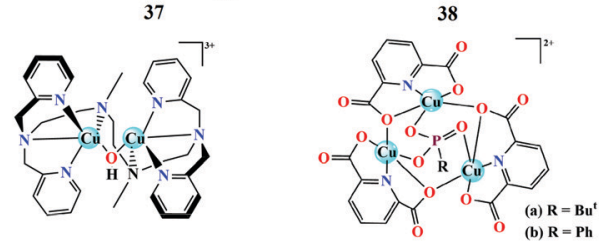

42

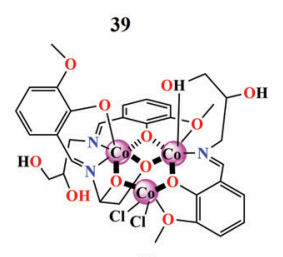

44

Fig. 4 Selected recently reported bi- and trinuclear WOCs discussed in Sections 1.2.2 and 1.2.3.

Proceeding to Co-based binuclear WOCs, their representatives are still limited, but are well characterized with respect to their mechanistic propagations. A representative example is the Co-Hbpp complex, which was initially reported for the fourelectron reduction of $\mathrm{O}_{2}$, leading to the formation of binuclear $\mu$-peroxo species 27 as a result of PCET processes. ${ }^{98}$ More recently, combined spectroscopic analysis of 27 and isolation of the corresponding Co superoxido end-on $\left(\left(\mathrm{H}_{2} \mathrm{O}\right)-\mathrm{Co}^{\mathrm{III}}\right.$ $\left.\mathrm{Hbpp}-\mathrm{Co}^{\mathrm{III}}-\mathrm{OO}^{\bullet}\right)$ species revealed its potential to catalyse water oxidation through the Co superoxido end-on intermediate following a number of sequential oxidations. ${ }^{99}$ Complex 27 containing oxygen isotopologues upon oxidation clearly displayed the resonance Raman (rR) vibrational features of the superoxido group at $1121 \mathrm{~cm}^{-1}, 1088 \mathrm{~cm}^{-1}$ and $1055 \mathrm{~cm}^{-1}$, which correspond to ${ }^{16} \mathrm{O}-{ }^{16} \mathrm{O},{ }^{17} \mathrm{O}-{ }^{17} \mathrm{O}$ and ${ }^{18} \mathrm{O}-{ }^{18} \mathrm{O}$, respectively. An EPR signal was observed for $\left(\left(\mathrm{H}_{2} \mathrm{O}\right)-\mathrm{Co}^{\mathrm{III}}-\mathrm{Hbpp}-\mathrm{Co}^{\mathrm{III}}-\mathrm{OO}^{\bullet}\right)$ owing to the presence of an unpaired electron from the superoxido group. Furthermore, both of the above observations were in good accordance with the XANES spectra of the superoxido complex, which showed a positive edge shift of $0.4 \mathrm{eV}$ and multiplet feature at the pre-edge region compared to that of 27 , suggesting a change in the local geometry and electron density around the Co metal center. The above experimental observations in combination with DFT calculations were useful in identifying the catalytic pathways of 27 . Similarly, the $\mathrm{Co}^{\text {III- }}$ ( $\mu$-peroxo- $\mu$-hydroxo)-Co ${ }^{\text {III }}$ diastereomers 28 and 29 reported by
Kojima et al. depict another example of Co-based binuclear WOCs. ${ }^{100}$ At pH 7, cis diastereomer 29 showed higher activity compared to $\mathbf{2 8}$, indicating the importance of structural symmetry in catalytic intermediates. The conclusions from the Pourbaix diagram suggested that the isomer-based activity originated from the slightly negative oxidation potential of the one-electron oxidised 29 compared to 28. Characterization of the oneelectron oxidised species of 28 and 29 by EPR and Raman spectroscopy indicated the generation of a $\mathrm{Co}^{\text {III-}}$-( $\mu$-superoxo)$\mathrm{Co}^{\mathrm{III}}$ dimer, which was confirmed by the presence of a 15-line EPR superhyperfine splitting and superoxo $\mathrm{O}-\mathrm{O}$ vibrational mode, respectively. This suggested the plausible involvement of 28 and 29 as intermediates for the water oxidation catalyzed by the earlier reported Co-TPA dimer (TPA: tris(2-pyridylmethyl)-amine). ${ }^{101}$ However, later reports indicated that the formation of $\mathrm{CoO}_{x}$ can drive heterogeneous water oxidation instead of 29. ${ }^{102,103}$ Recently, Goswami et al. synthesized mixed valence $\mathrm{Co}^{\mathrm{II}} \mathrm{Co}^{\mathrm{III}}$ binuclear complex 30, which was shown to be an active water oxidation catalyst owing to the presence of aqua ligands on one Co center. ${ }^{104}$ Preliminary catalytic studies based on cyclic voltammetry indicated an increase in catalytic current with $\mathrm{pH}$ over the range of 7-11, which was attributed to the conversion of the aqua to a hydroxide ligand at higher $\mathrm{pH}$, as validated by the $\mathrm{d}-\mathrm{d}$-band changes in the UV-vis spectra.

Regarding Fe, which is the most abundant transition metal, the spectrum of Fe-based binuclear WOCs has witnessed great 
progress in the past five years. ${ }^{95,105}$ Experimental evidence from many Fe-based WOCs suggests that they generally involve $\mathrm{Fe}^{\mathrm{IV}}=\mathrm{O} / \mathrm{Fe}^{\mathrm{V}}=\mathrm{O}$ high-valent species as intermediates. ${ }^{106,107}$ As reported by Ma et al., the $\mu$-(oxo)- $\mu$-(acetato) form of di-iron complex 31 is the active species responsible for water oxidation compared to its monomer counterpart $\left[\mathrm{Fe}(\mathrm{TPA})\left(\mathrm{Cl}_{2}\right)\right]$ at $\mathrm{pH}$ 4.5. ${ }^{108} \mathrm{~A}$ high-valent $\mathrm{Fe}^{\mathrm{IV}}=\mathrm{O}$ intermediate was assumed to be involved in the catalytic cycle, as confirmed from the evolution of a short-lived UV-vis peak at $726 \mathrm{~nm}$ upon the addition of oxone. By the targeted synthesis of 33, Åkermark et al. demonstrated the need for a proper ligand framework and nuclearity effects for Fe-based systems to function as efficient catalyts. ${ }^{109} \mathrm{Fe}-\mu$-(oxo) dimers based on polypyridyl macrocyclic ligand frameworks have recently been shown to serve as enhanced WOCs. ${ }^{110,111}$ Among them, the binuclear $\mathrm{Fe}_{2}-\mu$-(oxo)(ppq) complex 32 (ppq: 2-(pyrid-2'-yl)-8-(1"1,10"-phenanthrolin-2"-yl)quinoline) drives water oxidation in the presence of ceric ammonium nitrate. ${ }^{110}$ Electrochemical and UV-vis absorption studies suggest that water oxidation by $\mathbf{3 2}$ involves a simultaneous two-electron oxidation process, producing an $\mathrm{Fe}^{\mathrm{III}} \mathrm{Fe}^{\mathrm{V}}=\mathrm{O}$ intermediate owing to the presence of multiple redox peaks and an absorption band at $675 \mathrm{~nm}$. Interestingly, the $\mathrm{Fe}_{2}-\mu$-(oxo)(Hbbpya) $)_{2}$ complex 34 showed the influence of the electrode surface on the reaction mechanisms of homogenous water oxidation electrocatalysts. ${ }^{111}$ The mechanistic insight for $\mathbf{3 4}$ suggested the regeneration of the $\mathrm{Fe}_{2}-\mu-($ oxo $)(\text { Hbbpya })_{2}$ dimer from two monomeric $\mathrm{Fe}^{\mathrm{IV}} / \mathrm{Fe}^{\mathrm{V}}$ moieties over the catalytic turnover, thus confirming the $\mu$-(oxo) dimer $\mathbf{3 4}$ to be the real catalyst. Recently, metallocorroles have been well established as promising candidates for water oxidation due to the electronic stability offered by the corrole framework..$^{52,112}$ The binuclear Fe corroles 36 and 37 exhibit OER activity, with 37 displaying higher performance. This was attributed to the presence of a $\beta-\beta^{\prime}$ linkage in the latter, which modulates the electronic factors between the two Fe centers by promoting their simultaneous participation compared to the $\mu$-(oxo)-linked 36. Even though the majority of Fe-based WOCs involve high-valent $\mathrm{Fe}^{\mathrm{IV}} / \mathrm{Fe}^{\mathrm{V}}$ species in their catalytic turnovers, a recent report from the Das group suggested $\mathrm{O}_{2}$ evolution from dimeric Fe complex $\mathbf{3 5}$ as a consequence of the replacement of labile chlorides by hydroxide ions, leading to the formation of a peroxo-bridged Fe ${ }^{\mathrm{III}}$ species. ${ }^{113}$

In accordance with the fact that $\mathrm{Cu}-$ oxo dimers play an active role in several redox processes in nature, the development of Cu-based WOCs has been a topic of great interest over the past decade due to the well-defined redox behaviour and relative earth abundance of $\mathrm{Cu}^{85,114}$ With the report of 38 in 2015 , a totally new pathway for water oxidation catalyzed by binuclear $\mathrm{Cu}$ systems was proposed, which was otherwise believed to involve high-valent $\mathrm{Cu}^{\mathrm{IV}}=\mathrm{O}$, as in the case of monomers. ${ }^{115}$ The $\mathrm{Cu}$ dimer 38 catalyses water oxidation at neutral $\mathrm{pH}$, following an intramolecular proton transfer mechanism between the two $\mathrm{Cu}^{\mathrm{III}}$ centers by passing through $\mathrm{a} \mathrm{Cu}_{2}{ }^{\mathrm{II}, \mathrm{II}}$ superoxide intermediate. Subsequently, an unsymmetrical binuclear $\mathrm{Cu}$ complex 39 based on the earlier WOC demonstrated the key role of the intramolecular proton acceptor in the catalytic mechanism of 38 since 39 showed a buffer concentration-dependent catalysis, indicating the requirement of buffer to mediate the proton transfer process. ${ }^{116}$ In 2017, Emmons et al. proposed a new pathway for catalytic water oxidation by $\mathbf{4 1}$. This redox isomerization (RI) mechanism, which was supported with the identification

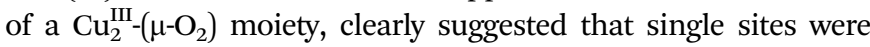
disfavoured during the corresponding catalytic pathway. ${ }^{60}$ Furthermore, calculations revealed that both the WNA and RI mechanisms are energetically probable pathways for $\mathrm{O}-\mathrm{O}$ bond formation. Very recently, Wang et al. succeeded in synthesizing a stable binuclear $\mathrm{Cu}$ core in WOC 42 using a suitable flexible linker group for the two metal centers. ${ }^{117}$ The electrochemical OER performance of $\mathbf{4 2}$ at $\mathrm{pH} 12$ displayed a 30-fold higher activity compared to its monomeric analogue. The preference of a flexible linker over a rigid aromatic linker was demonstrated to promote the intramolecular cooperative effect between the metal centers, thereby reducing the energy barrier for the RDS, as validated by DFT calculations.

1.2.3. Polynuclear WOCs. The design of polynuclear WOCs involves three major approaches. First, tetranuclear cubane complexes, second, the design of molecular metal complexes with higher nuclearities, and finally complete multi-nuclear, all-inorganic heteropolyoxometalates (POMs) with transition metal cores. One of the main advantages of polynuclear WOCs is their ability to distribute the oxidation equivalents over multiple metal centers, which can ultimately increase the efficiency of the OER at low overpotentials. ${ }^{118}$ However, it should also be noted that although not all tetra/polynuclear entities exhibit water oxidation properties, many of them can still serve as synthetic model systems for the better understanding the electronic structural features of the OEC. ${ }^{119}$ Recent analyses based on multidimensional magnetic resonance spectroscopy, EXAFS and quantum chemical modelling suggest the presence of both closed and open conformers for the OEC cubane core in different S states. ${ }^{120,121}$ In fact, these observations play an important role in designing artificial systems since they question the need for a rigid or flexible tetra metallic core in synthetic WOCs.

Earlier investigations in the development of cubane-type WOCs were solely concentrated on Mn-based systems owing to their presence in OEC and high natural abundance. ${ }^{122}$ Additionally, several cubane WOCs based on $\mathrm{Co}$ and $\mathrm{Cu}$ have been designed and investigated in the past decade. One of the most widely investigated Co cubane WOCs is $\mathrm{Co}_{4} \mathrm{O}_{4}(\mathrm{Py})_{4}(\mathrm{OAc})_{4}$, whose mechanistic and electronic features during water oxidation have been well determined recently via quantum chemical modelling and advanced spectroscopic techniques. Chen $e t$ al. characterized the possible high-valent intermediates in the OER catalyzed by $\mathrm{Co}_{4} \mathrm{O}_{4}(\mathrm{Py})_{4}(\mathrm{OAc})_{4}$ cubane using XAS and $\mathrm{K} \beta$ RIXS coupled with DFT calculations. ${ }^{123}$ The formation of highvalent $\mathrm{Co}(\mathrm{Iv})$ species during the OER has been previously identified in $\mathrm{Co}_{4} \mathrm{O}_{4}$ cubane complexes and Co-Pi via multifrequency EPR spectroscopy. ${ }^{124,125}$ Owing to the faster timescale of the electronic transitions in X-ray spectroscopic methods compared to EPR, XAS/RIXS can be used as a valuable technique to probe the localized high-valent $\mathrm{Co}(\mathrm{Iv})$ state. The combined XAS/RIXS analysis confirmed that the hole equivalents 
were stored within the cubane core, leading to the evolution of a Co(Iv) state. Furthermore, in contrast to the previous EPRbased conclusions, these recent X-ray spectroscopy investigations indicated that the hole equivalents were localized rather than delocalized within the cubane cluster. ${ }^{123}$ Furthermore, these observations were supported by DFT calculations, which suggested the futility of electron hole delocalization for the formation of $\mathrm{Co}(\mathrm{Iv})$ in clusters. Additionally, calculations based on hypothetical $\mathrm{CoM}_{3} \mathrm{O}_{4}$ cubanes formed from the substitution of redox-inactive metals $(\mathrm{M})$ indicated the facilitated formation of localized $\mathrm{Co}(\mathrm{Iv})$. Further studies on modelling the Co-Pi active sites based on the cofacial $\mathrm{Co}(\mathrm{Iv})$ sites of $\mathrm{Co}_{4} \mathrm{O}_{4}(\mathrm{Py})_{4}{ }^{-}$ $(\mathrm{OAc})_{4}$ cubane have also been carried out recently to understand and correlate the formation of high-valent Co species in Co-based 0WOCs. ${ }^{126}$ This has been helpful in concluding the need for adjacent $\mathrm{Co}(\mathrm{Iv})$ sites in oxidic cobalt WOCs to drive $\mathrm{O}-\mathrm{O}$ bond formation. The in situ rapid-scan FT-IR spectroscopic investigations by Frei and co-workers indicated that the photo-driven water oxidation by $\mathrm{Co}_{4} \mathrm{O}_{4}(\mathrm{Py})_{4}(\mathrm{OAc})_{4}$ cubane involves a $\mathrm{Co}^{\mathrm{III}}-(\mu-$ peroxide)-Co ${ }^{\mathrm{III}}$ species at the $\mathrm{O}-\mathrm{O}$ bond formation step, which is preceded by a $\mathrm{Co}^{\mathrm{III}} \mathrm{Co}^{\mathrm{IV}}$ intermediate. ${ }^{127}$ In 2017 , our group reported a highly intact and stable Co-dpk (dpk: di(2-pyridyl)ketone) cubane WOC 45 (Fig. 5), displaying the characteristic $\left\{\mathrm{H}_{2} \mathrm{O}-\mathrm{Co}_{2}(\mathrm{OR})_{2}-\mathrm{OH}_{2}\right\}$ edge-site motif that catalyses photo-driven water oxidation. ${ }^{26}$ In situ XANES investigations of $\mathbf{4 5}$ suggested the oxidation of the $\mathrm{Co}$ (II) centers to $\mathrm{Co}$ (III) or higher valent states during the catalytic turnover. DFT calculations indicated the role of the abovementioned edge-site aqua ligands in mediating the proton transfer process. Another work from our group involved the incorporation of $\mathrm{Ln}^{3+}$ as redox-inactive centers within the cubane core of a previously reported Co cubane WOC. ${ }^{128}$ The role of $\mathrm{Ln}^{3+}$ in 46, as a redox-inactive $\mathrm{Ca}^{2+}$ analogue of OEC, was depicted as an alternative strategy in the design of active

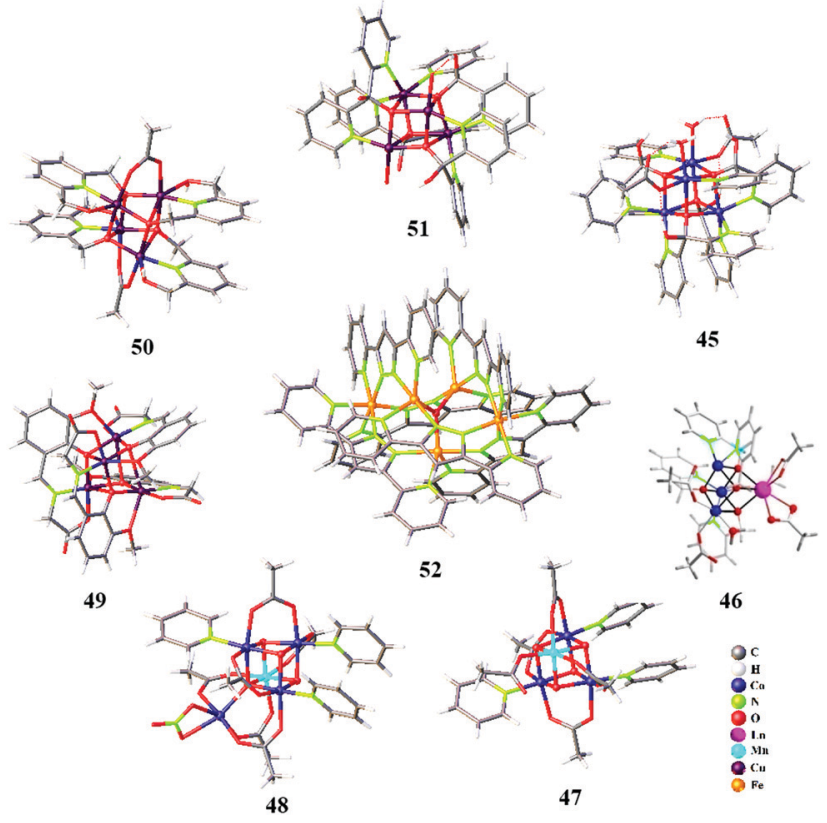

Fig. 5 Selected recent polynuclear WOCs discussed in Section 1.2.3. cubane WOCs. Furthermore, the $\mathrm{Ln}^{3+}$ center also served as a catalytic promoter site by offering a flexible ligand-binding motif. Carrying forward the investigations on derivatives of $\mathrm{Co}_{4} \mathrm{O}_{4}(\mathrm{Py})_{4}(\mathrm{OAc})_{4}$, Tilley et al. established the role of heterometal incorporation and dangling metal sites on cubane cores in tuning the catalytic activity. ${ }^{129,130}$ Insertion of $\mathrm{Mn}$ into the cubane core led to the formation of $\mathrm{MnCo}_{3} \mathrm{O}_{4}(\mathrm{Py})_{3}(\mathrm{OAc})_{5} \mathbf{4 7}$, which was introduced as a molecular analogue for Mn-doped Co oxidic WOCs. The spectroscopic and electrochemical investigations suggested that the redox chemistry occurs at the Co sites, whose redox behaviours are tuned by the ligand attached on $\mathrm{Mn}$, thus establishing a cooperative effect between Mn and Co. ${ }^{129}$ This in fact serves as a valuable strategy towards the understanding and design of heterobimetallic catalysts. Furthermore, attachment of a dangling Co site to the $\mathrm{MnCo}_{3} \mathrm{O}_{4}(\mathrm{Py})_{3}(\mathrm{OAc})_{5}$ cubane moiety resulted in the formation of 48. This synthetic analogue of the OEC indicated the role of a dangling metal center in modulating the structural rigidity and redox behaviour of the cubane core. Crystallographic information supported by computational data clearly suggested the distortion of the cubane core upon the addition of the dangling center. Furthermore, $\mathbf{4 8}$ shows an increased number of redox events compared to the parent cubane 47 , thus indicating the productive role of the dangling metal center in facilitating multielectron processes. ${ }^{130} \mathrm{Wu}$ et al. for the first time reported the $\mathrm{Cu}$ cubane 49 , which serves as a water oxidation electrocatalyst. Mechanistic investigations based on in situ EPR spectroscopy suggested the formation of an EPR silent lowspin $\mathrm{Cu}^{\mathrm{III}}$ species, which is involved in the catalytic turnover during bulk electrolysis. ${ }^{131}$ This was further supported by the observation of $\mu_{4}$-peroxido $\mathrm{O}-\mathrm{O}$ vibrational features in the Raman spectra. Therefore, it was concluded that the mechanistic intermediates involved were low-spin $\mathrm{Cu}^{\mathrm{III}}$ and $\mathrm{Cu}^{\mathrm{III}} \mathrm{O}$ species formed as a result of the successive two-electron oxidation of the initial $\mathrm{Cu}_{4}^{\text {II }}$ species. ${ }^{131}$ Another tetranuclear $\mathrm{Cu}$ cubane WOC $\mathbf{5 0}$ was reported by Wang et al., which serves as a stable electrocatalyst over a broad $\mathrm{pH}$ range of 8.92-11.78 with a relatively low overpotential of $400-665 \mathrm{mV} .^{132}$ The mechanistic pathway was suggested to follow a PCET mechanism, as supported by the linear dependence of the catalytic water oxidation potentials on the $\mathrm{pH} .{ }^{132}$ In 2018, Ding et al. reported an octanuclear $\mathrm{Cu}$-based WOC comprising two $\mathrm{Cu}_{4} \mathrm{O}_{4}$ cubane units 51 supported by dpk ligands. This WOC served as an efficient catalyst for the photo-driven oxidation of water in the presence of the $\left[\mathrm{Ru}(\mathrm{bpy})_{3}\right]^{2+}$ photosensitizer. ${ }^{133}$ The plausible mechanism was suggested to involve the pre-organization of solvent water molecules via hydrogen bonding from the $\mathrm{dpk}-\mathrm{OH}^{-}$ligands, thus favouring the nucleophilic attack of water on $\mathrm{Cu}^{\mathrm{II}}$. Besides the tetranuclear cubane-type WOCs, trinuclear metal complexes based on $\mathrm{Cu} 43$ and Co 44 have recently been reported as efficient WOCs. ${ }^{105,134}$

Besides tetranuclear cubanes and trinuclear WOCs, very few metal complexes of higher nuclearity have been reported as efficient WOCs. A representative well-investigated polynuclear WOC is the pentanuclear Fe complex 52, consisting of a triangular $\left[\mathrm{Fe}_{3}\left(\mu_{3}-\mathrm{O}\right)\right]$ core surrounded by two $\left[\mathrm{Fe}(\mu-\mathrm{L})_{3}\right]$ units, 
thus possessing open coordination sites. ${ }^{135}$ Earlier mechanistic insights proposed that the $\mathrm{O}-\mathrm{O}$ bond formation proceeds through the coupling of two $\mathrm{Fe}^{\mathrm{IV}}=\mathrm{O}$ units within the $\mathrm{Fe}_{2}^{\mathrm{II}} \mathrm{Fe}^{\mathrm{III}} \mathrm{Fe}_{2}^{\mathrm{IV}}$ cluster. However, a recent investigation based on DFT calculations on the same Fe system suggested a different and more energetically plausible pathway. The new mechanism is based on the involvement of a superoxide $\mathrm{Fe}_{5}^{\mathrm{III}}$ intermediate prior to $\mathrm{O}-\mathrm{O}$ bond formation rather than the participation of ferrous sites. ${ }^{136}$ Another polynuclear Mn cluster with $12 \mathrm{Mn}$ atoms was recently reported by the Christou group. The $\mathrm{Mn}_{12}$ dhbH (dhbH: 3,5-dihydroxybenzoic acid) cluster serves as a water oxidation electrocatalyst at $\mathrm{pH} 6 .^{137}$ The specific ligand design with carboxylate groups endows the $\mathrm{Mn}_{12}$ cluster with water solubility and stability. The multinuclear metal sites contribute to the catalytic activity of the cluster, which is also supported by its multiple redox behaviour. Moreover, it was suggested that the hydroxyl groups serve as hydrogen bonding sites, thus enabling a shuttle pathway for $\mathrm{H}_{2} \mathrm{O}$ and $\mathrm{H}^{+}$to and from the Mn active sites.

As mentioned earlier, the design of polyoxometalates with multinuclear TM metal cores is also important for the development of WOCs. POMs as structurally rigid and stable molecular metal oxo clusters have been well recognized as efficient WOCs owing to their capability to drive fast and reversible multi-electron transfer transformations, while preserving their structural motifs. ${ }^{138-140}$ POMs incorporating tetranuclear Co and trinuclear $\mathrm{Cu}$ metal sites have been identified as potential water oxidation photo- and electrocatalysts, respectively. Besides these smaller representatives, giant POMs with higher nuclearity have also been identified as stable WOCs. One such $\mathrm{POM}$ is the $\left[\mathrm{Mo}_{72} \mathrm{Fe}_{30} \mathrm{O}_{252}\left(\mathrm{CH}_{3} \mathrm{COO}\right)_{12}\left\{\mathrm{Mo}_{2} \mathrm{O}_{7}\left(\mathrm{H}_{2} \mathrm{O}\right)\right\}_{2}\left\{\mathrm{H}_{2} \mathrm{Mo}_{2} \mathrm{O}_{8}{ }^{-}\right.\right.$ $\left.\left(\mathrm{H}_{2} \mathrm{O}\right)\right\}\left(\mathrm{H}_{2} \mathrm{O}\right)_{91}$ ] oxo-cluster incorporating $30 \mathrm{Fe}$ cores, which catalyses water oxidation under photocatalytic conditions at pH 9. ${ }^{141}$ For a more detailed discussion of POM-based water splitting catalysts, we would like to recommend recent representative reviews. ${ }^{142,143}$

As evident from the above discussion, molecular compounds offer a wide variety of options in the strategic design of efficient WOCs. The choice of a suitable ligand framework is essential, while targeting a multinuclear WOC favours the synergistic effects of metal sites and other electronic modulations via the ligand to enhance efficient hydrogen bonding interactions. Although combined spectroscopic techniques serve as a toolbox for the mechanistic investigations of molecular WOCs, they are sometimes unable to fully capture the chemical dynamics of molecules in solutions. Thus, future research strategies on molecular WOCs will move towards their immobilization on heterogeneous surfaces to avoid the limitations of conventional solution-based approaches. ${ }^{144-146}$

\subsection{The importance of molecular integrity}

1.3.1. Molecular integrity as an indispensable feature for mechanisms. Under harsh reaction conditions, e.g. high overpotential and strong basic media, some molecular WOCs become unstable and decompose into metal compounds, such as metal oxides, which then act as the true catalyst. ${ }^{15}$
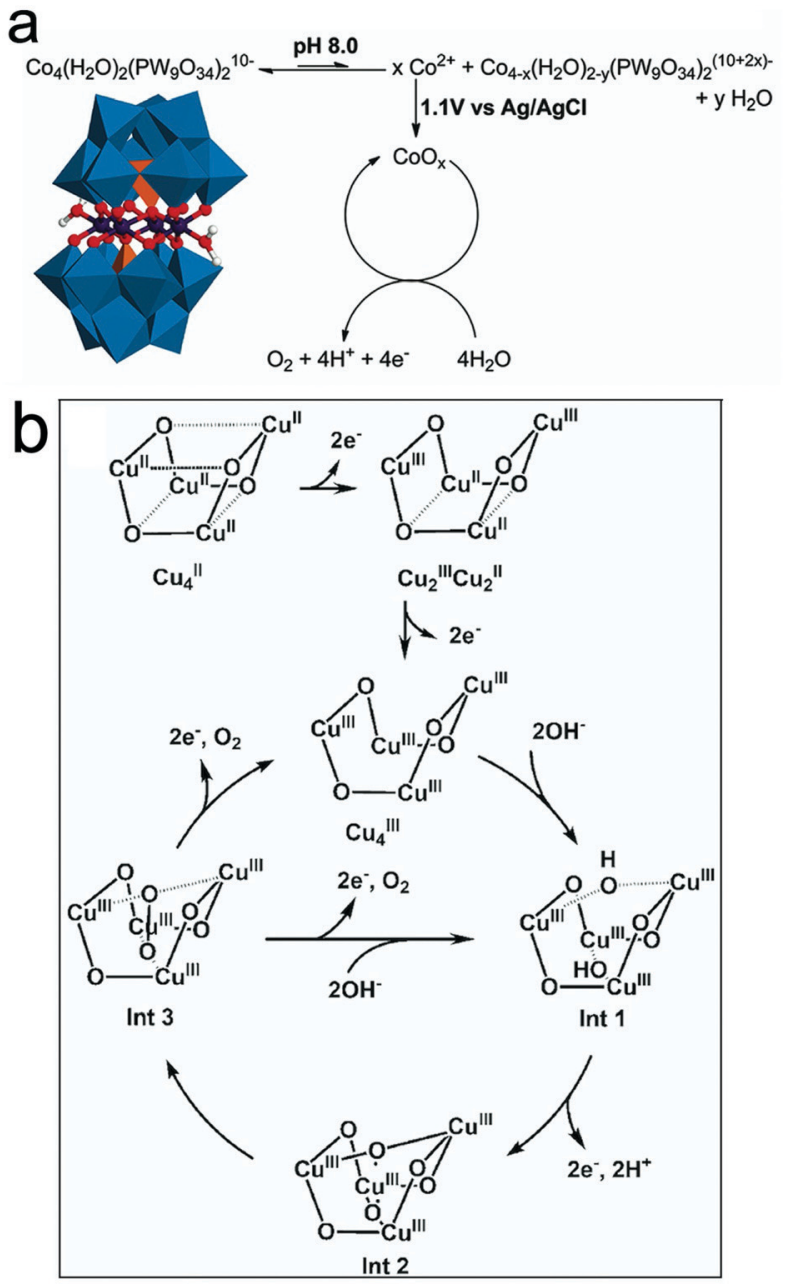

Fig. 6 (a) Heterogeneous $\mathrm{CoO}_{x}$ formation from homogeneous $\mathrm{CO}_{4} \mathrm{POM}$. Reproduced with permission from ref. 148. Copyright 2011, The American Chemical Society. (b) Mechanism for the water oxidation of the $\mathrm{Cu}_{4} \mathrm{O}_{4}$ core for $\left[(\mathrm{LGly}-\mathrm{Cu})_{4}\right] /\left[(\mathrm{LGlu}-\mathrm{Cu})_{4}\right]$ in alkaline solution. Reproduced with permission from ref. 131. Copyright 2018, Wiley VCH.

For mechanistic investigations, establishing the integrity of molecular WOCs is indispensable because the correct assignment of kinetics, atomic environment, electron/proton transfer, and binding energy all depend on the nature and structure of the true catalyst. Therefore, great care must be taken to identify the true active species in molecular WOCs. In a classic example, previous studies reported $\left[\mathrm{Co}_{4}\left(\mathrm{H}_{2} \mathrm{O}\right)_{2}\left(\mathrm{PW}_{9} \mathrm{O}_{34}\right)_{2}\right]^{10-}\left(\mathrm{Co}_{4} \mathrm{POM}\right)$ as a stable homogeneous WOC. ${ }^{147}$ Later, revisions of the molecular integrity of this WOC showed that $\left(\mathrm{Co}_{4} \mathrm{POM}\right)$ could decompose into amorphous $\mathrm{CoO}_{x}$ under electrochemical conditions, which acts as a heterogeneous WOC. Both $\mathrm{Co}_{4} \mathrm{POM}$ and $\mathrm{CoO}_{x}$ can contribute to the catalytic activity, with $\mathrm{Co}_{4} \mathrm{POM}$ being the dominant WOC (Fig. 6a). ${ }^{148-151}$

However, more recent studies confirmed the molecular integrity of many molecular WOCs. Particularly, studies on the structural integrity of $\mathrm{Cu}$-containing $\left[\mathrm{V}_{2} \mathrm{Mo}_{6} \mathrm{O}_{26}\right]^{6-} \mathrm{POM}-$ WOCs $^{152}$ showed that these POMs do not exhibit nanoparticle growth (0.04 M borate buffer, $\mathrm{pH}$ 8) based on DLS experiments. 
Regarding the Cu-sites, their high activity and low overpotential are controlled by their coordination environment. The first oneelectron oxidation leads to the formation of $\mathrm{Cu}^{\mathrm{III}}-\mathrm{OH}$ species followed by $\mathrm{Cu}^{\mathrm{IV}}=\mathrm{O}$ oxidation, which assists in nucleophilic attack, while the aqua-oxo interaction favour the formation of intramolecular $\mathrm{O}-\mathrm{O}$ bonds. ${ }^{152}$ Other works on the molecular integrity of Cu-cubane $\left[\left(\mathrm{L}_{\mathrm{Gly}}-\mathrm{Cu}\right)_{4}\right] /\left[\left(\mathrm{L}_{\mathrm{Glu}}-\mathrm{Cu}\right)_{4}\right]$ in alkaline solution ${ }^{131}$ indicated that the structure of the $\mathrm{Cu}_{4} \mathrm{O}_{4}$ core remains intact, with no oxide formation during the OER. The two-electron transfer processes involved in the tetranuclear $\mathrm{Cu}_{4} \mathrm{O}_{4}$-cubane centers yield high-valent $\mathrm{Cu}^{\mathrm{III}} / \mathrm{Cu}^{\mathrm{III}} \mathrm{O}^{\bullet}$ intermediates during the OER (Fig. 6b). Other recent studies on the true catalyst in the Mn-containing $\left[\mathrm{Mn}_{3}\left(\mathrm{H}_{2} \mathrm{O}\right)_{3}\left(\mathrm{SbW}_{9} \mathrm{O}_{33}\right)_{2}\right]^{12-}$ POM showed that no $\mathrm{Mn}^{2+} / \mathrm{Mn}$-oxide species are formed under water oxidation conditions. ${ }^{153}$ The Sb heteroatoms in the polyoxoanions were found to play a central role in their stability during the water oxidation process.

1.3.2. Stabilization of molecular WOCs. During the past years, a number of high-performance molecular WOCs with manifold active units based on noble and non-noble earth abundant metals have been successfully synthesized. ${ }^{38,154}$

However, the loss of structural integrity under harsh chemical oxidants or in the presence of sacrificial reagents remains a problem for molecular WOCs. Also, electrode fabrication from molecular WOCs by drop casting can lead to catalyst leakage, high aggregation, and inefficient activation of metal centers at the interfacial diffusion layer. ${ }^{15}$ Thus, to overcome these issues, novel strategies for the heterogeneous stabilization of molecular WOCs on solid surfaces have been developed. ${ }^{155}$ The main goals include (i) preserving the intrinsic activity and selectivity, (ii) stabilization of intermediate species, and lowering the energy barrier while facilitating $\mathrm{O}-\mathrm{O}$ bond formation, (iii) tuning the catalyst loading to lower the water oxidation overpotentials, (iv) attaining facile electron transfer kinetics at the interface, and (v) promoting long-term resilience against leaching and highly oxidative media. ${ }^{15,23,38}$ All this must be complemented with the optimization of surface area/ morphology, hydrophobicity and hydrophilicity, density of active metal sites, and mass/charge transfer properties. Particularly, the stabilization of molecular WOCs by tuning the electronic/steric properties of the ligand requires meticulous screening since the nature of the ligand can affect their activity and selectivity. ${ }^{155,156}$ Recently, $N, N^{\prime}$-bidentate electronically asymmetric ligands were used to stabilize low-valent transient $\mathrm{Co}^{\mathrm{I}}$ species. ${ }^{157}$ The results showed that electron-deficient ligands removed electron density from the Co sites, thereby preventing deactivation of the catalyst, and stabilizing the complex (Fig. 7a). Similarly, mononuclear $[\mathrm{Co}(\mathrm{TPA}-\mathrm{R})]^{2+}(\mathrm{TPA}=\operatorname{tris}(2$-pyridylmethyl)amine, $\mathrm{R}=$ tri- $\alpha \mathrm{F}$; tri$\alpha \mathrm{OMe}$ and mono- $\alpha \mathrm{F}$ ) was stabilized through electron-withdrawing substituents on the TPA ligand. ${ }^{76}$ The oxidative resistance of the ligand on Co-intermediates was found to be a key factor to achieve oxidative stability. Mn-porphyrin dimer WOCs fabricated with mono/hexaphosphonic acid groups $\left(\mathrm{Mn}_{2} \mathrm{DP}-\mathrm{PO}_{3} \mathrm{H}_{2}\right.$ and $\mathrm{Mn}_{2} \mathrm{DP}-$ $\left.\left(\mathrm{PO}_{3} \mathrm{H}_{2}\right)_{6}\right)$ were recently immobilized through covalent assembly on ITO electrodes. ${ }^{158}$ The immobilized $\mathrm{Mn}_{2} \mathrm{DP}-\left(\mathrm{PO}_{3} \mathrm{H}_{2}\right)_{6} \mid \mathrm{ITO}$ assists electron transfer from the WOC to the ITO-electrode, allowing efficient OER at a low overpotential with high turnover. Similarly, Co-complexes have been stabilized through the covalent anchoring of 2,2-bipyridine to graphene-modified ITO electrodes (Fig. 7b). ${ }^{159}$ The heterogenized system yields a surface-bound
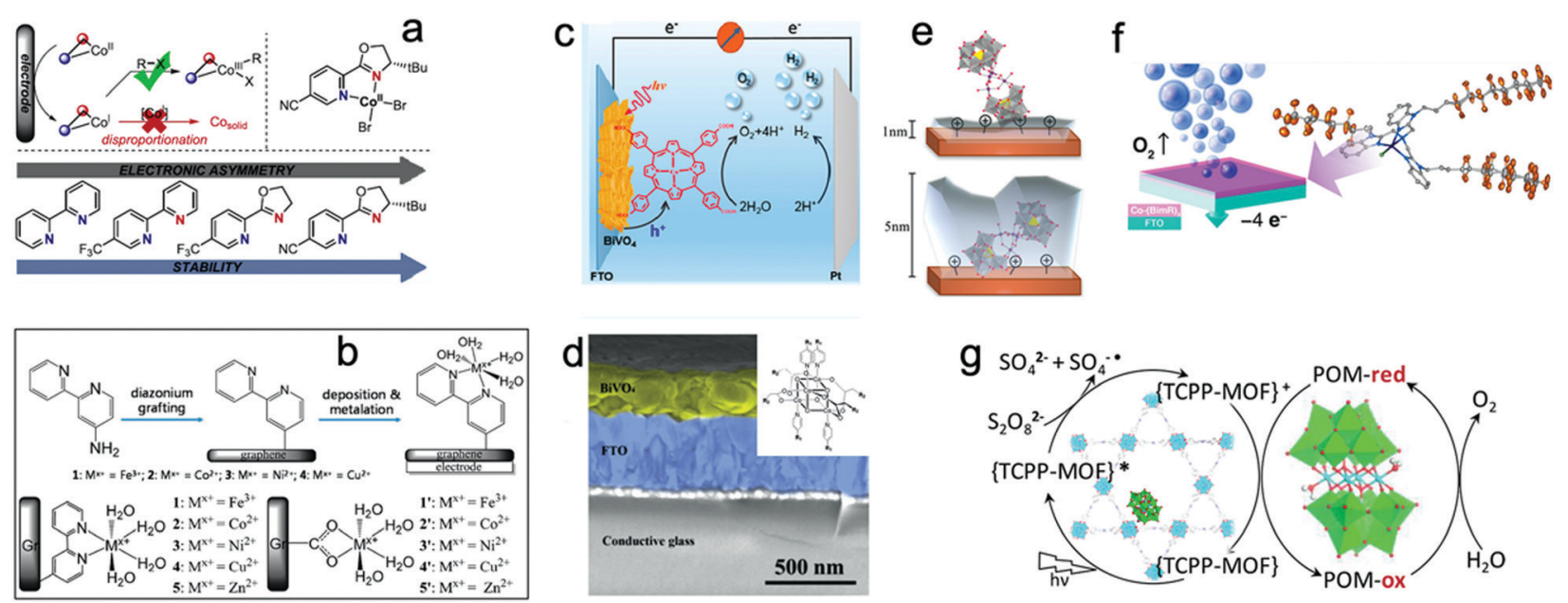

Fig. 7 (a) Stabilization of a low-valent transient Co' complex by using $N, N^{\prime}$-bidentate electronically asymmetric ligands. Reproduced with permission from ref. 76. Copyright 2017 Elsevier. (b) TM complexes ( $\left.M=\mathrm{Fe}^{3+}, \mathrm{Co}^{2+}, \mathrm{Ni}^{2+}, \mathrm{Cu}^{2+}, \mathrm{Zn}^{2+}\right)$ immobilized by covalent anchoring of 2,2-bipyridine to Gr-modified ITO electrodes. Reproduced with permission from ref. 159. Copyright 2014 The American Chemical Society. (c and d) Stabilization of molecular porphyrin complexes CoTCPP, and molecular $\mathrm{CO}_{4} \mathrm{O}_{4}$ cubane type WOCs on $\mathrm{BiVO}_{4}$ photoanodes. Reproduced with permission from ref. 161 and 162. Copyright 2016 The American Chemical Society and copyright 2017 Wiley VCH, respectively. (e). Stabilization of an $\mathrm{Al}_{2} \mathrm{O}_{3}-\mathrm{Coated}$ [ $\mathrm{Ru}_{4}^{\mathrm{IV}}$ $\left.\left.(\mathrm{OH})_{2}\left(\mathrm{H}_{2} \mathrm{O}\right)_{4}\right\}\left(\gamma-\mathrm{SiW}_{10} \mathrm{O}_{34}\right)_{2}\right]^{10-}$ POM on hematite photoelectrodes. Reproduced with permission from ref. 164. Copyright 2017 The American Chemical Society. (f) Stabilization of molecular Co-WOCs enclosing tris((2-benzimidazolymethyl)amine) ligands, $\mathrm{Co}_{-}\left(\mathrm{BimC}_{3} \mathrm{~F}_{8}\right)_{3}$, on the surface of an FTO electrode. Reproduced with permission from ref. 165. Copyright 2016 The American Chemical Society. (g) Stabilization of $\left[\mathrm{CO}_{4}\left(\mathrm{H}_{2} \mathrm{O}_{2}\left(\mathrm{PW}_{9} \mathrm{O}_{34}\right)_{2}\right]^{10-}\right.$ polyoxometalate into the hexagonal channels of the $\mathrm{Zr}(\mathrm{Iv})$ porphyrinic MOF-545 hybrid framework. Reproduced with permission from ref. 166. Copyright 2018 The American Chemical Society. 
WOC with only covalently attached active catalytic species, displaying high catalytic activity. Thus, functionalization of graphene-modified electrodes offers a viable route for loading metal atoms on conductive surfaces with appropriate metal binding moieties. In a similar study, a molecular $\left[\left(\mathrm{L}_{\mathrm{py}}\right) \mathrm{Cu}^{\mathrm{II}}\right]^{2-}$ complex ( $\mathrm{L}_{\mathrm{py}}=$ 4-pyrenyl-1,2-phenylenebis(oxamidate) ligand) was stabilized on graphene electrodes. ${ }^{160}$ Anchoring promotes $\pi$-stacking interactions with the graphene layers, which provide $\pi$-delocalization to notably enhance the catalytic performance. The molecular $\left[\left(\mathrm{L}_{\mathrm{py}}\right) \mathrm{Cu}^{\mathrm{II}}\right]^{2-}$ WOCs remained intact under catalytic turnover without any sign of $\mathrm{CuO}$ formation. Recently, cobalt porphyrin CoTCPP and $\mathrm{Co}_{4} \mathrm{O}_{4}$ cubane WOCs were stabilized on $\mathrm{BiVO}_{4}$ photoanodes (Fig. 7c and d; also see Part III). ${ }^{161,162}$ The results revealed that CoTCPP suppresses electron-hole recombination on the $\mathrm{BiVO}_{4}$ surface when using $\mathrm{H}_{2} \mathrm{O}_{2}$ as a hole scavenger, while improving both activity and stability. Loading $\mathrm{Co}_{4} \mathrm{O}_{4}$ cubane featuring alkoxycarboxylato bridging ligands on porous $\mathrm{BiVO}_{4}$ electrodes also resulted in highly efficient surface charge separation.

Stabilization through covalent functionalization, namely modification of electrode surfaces with functional groups, has been used to stabilize tetranuclear $\left[\mathrm{Ru}_{4}^{\mathrm{IV}} \mathrm{O}_{5}(\mathrm{OH})\left(\mathrm{H}_{2} \mathrm{O}\right)_{4}(\gamma-\right.$ $\left.\left.\mathrm{PW}_{10} \mathrm{O}_{36}\right)_{2}\right]^{9-}$ and $\left[\left\{\mathrm{Ru}_{4}^{\mathrm{IV}}(\mathrm{OH})_{2}\left(\mathrm{H}_{2} \mathrm{O}\right)_{4}\right\}\left(\gamma-\mathrm{SiW}_{10} \mathrm{O}_{34}\right)_{2}\right]^{10-}$ POMWOCs on porous $\mathrm{TiO}_{2}$ electrodes. ${ }^{163}$ Treatment of the $\mathrm{TiO}_{2}$ electrode with cationic silylating agents resulted in better stabilization of the POM-WOC on the surface of the $\mathrm{TiO}_{2}$ electrodes, where it remained intact after photo-driven water oxidation. Stabilization of $\left[\left\{\mathrm{Ru}_{4}^{\mathrm{IV}}(\mathrm{OH})_{2}\left(\mathrm{H}_{2} \mathrm{O}\right)_{4}\right\}\left(\gamma-\mathrm{SiW}_{10} \mathrm{O}_{34}\right)_{2}\right]^{10-}$ on $\mathrm{Fe}_{2} \mathrm{O}_{3}$ photoelectrodes was also reported. ${ }^{164}$ However, the attached $\mathrm{POM}$ on the $\mathrm{Fe}_{2} \mathrm{O}_{3}$ photoanode surface remained intact after water oxidation only if a protective $\mathrm{Al}_{2} \mathrm{O}_{3}$ layer was deposited on top the POM (Fig. 7e). Moreover, the thickness of the $\mathrm{Al}_{2} \mathrm{O}_{3}$ layer also drastically affected the catalytic performance of the attached POM-WOC. Another study reported the stabilization of Co-encapsulating $\mathrm{C}_{24} \mathrm{H}_{21} \mathrm{~N}_{7}$ ligands Co$\left(\text { BimC }_{3} \mathrm{~F}_{8}\right)_{3}$ on FTO electrodes (Fig. 7f). ${ }^{165}$ The stability of this immobilized molecular Co-WOC was found to depend on the tailored hydrophobicity of the coordinating ligand. The Co-WOC, which was immobilized by physisorption, remained stable during extended electrolysis.

Stabilization of molecular WOCs through physical confinement was used for the encapsulation of the $\left[\mathrm{Co}_{4}\left(\mathrm{H}_{2} \mathrm{O}\right)_{2}\left(\mathrm{PW}_{9} \mathrm{O}_{34}\right)_{2}\right]^{10-}$ POM in the hexagonal channels of a $\mathrm{Zr}(\mathrm{Iv})$ porphyrinic MOF-545 (Fig. $7 \mathrm{~g}$ ). ${ }^{166}$ This approach showed that the POM-MOF interface delivers - $\mathrm{OH}$ species and labile $\mathrm{H}_{2} \mathrm{O}$ molecules, which clearly enhance the OER performance. ${ }^{166}$ Similarly, encapsulation of Co-substituted polyoxometalates (Co-POMs), was performed via an impregnation route using mesoporous $\mathrm{Cr}$ (III)terephthalate MIL-101(Cr) MOFs by stirring a solution of Co-POM and MIL-101(Cr). ${ }^{167}$ The results from ${ }^{31} \mathrm{P}$ NMR and elemental analyses indicated that the $\left[\mathrm{Co}_{4}\left(\mathrm{H}_{2} \mathrm{O}\right)_{2}\left(\mathrm{PW}_{9} \mathrm{O}_{34}\right)_{2}\right]^{10-}$ and $\left[\mathrm{CoO}_{39}\left(\mathrm{H}_{2} \mathrm{O}\right) \mathrm{PW}_{11}\right]^{5-}$ POMs remained intact inside the MOF framework. A similar study encapsulated $\mathrm{Ni}_{4} \mathrm{P}_{2}-\mathrm{POM}\left[\mathrm{Ni}_{4}\left(\mathrm{H}_{2} \mathrm{O}\right)_{2}\right.$ $\left.\left(\mathrm{PW}_{9} \mathrm{O}_{34}\right)_{2}\right]^{10-}$ into the pores of MOFs obtained from $\left[\operatorname{Ir}(\mathrm{ppy})_{2}(\mathrm{bpy})\right]^{+}$and $\left[\mathrm{Ru}(\mathrm{bpy})_{3}\right]^{2+}$-derived dicarboxylate ligands. ${ }^{168}$ The results revealed that the proximity of $\mathrm{Ni}_{4} \mathrm{P}_{2}$ to the photosensitizer in $\mathrm{Ni}_{4} \mathrm{P}_{2} @ M O F$ assists multi-electron transfer for efficient catalysis. Hence, the hierarchically ordered POM@MOF assembly provides a tunable platform for achieving synergistic functionalization (also see Section 3.3.2).

Developing stabilization strategies for molecular WOCs remains a main challenge for large-scale applications. Accordingly, effective heterogenization of molecular WOCs is a promising method to achieve catalysts with enhanced atom-efficiency and intrinsic activity for large-scale artificial photosynthesis. Hence, further studies are needed for efficient device assembly with extended molecular stability, robustness and superior catalytic performance.

\section{Part II: development and mechanistic studies of heterogeneous WOCs}

\subsection{Mechanistic advances for heterogeneous WOCs}

Over the past decade, considerable efforts in the heterogeneous WOC community were devoted to the design of efficient catalysts with a higher TOF at minimized overpotentials. ${ }^{3,169}$ Consequently, many first-row bi- and multi-TM-based systems have already surpassed noble metal oxides such as $\mathrm{RuO}_{x}$ and $\mathrm{IrO}_{x}$ in terms of TOF. ${ }^{5,170-173}$ However, the ongoing debate on the involved active sites and the pathways of the fourelectron-transfer reaction still demand in-depth mechanistic investigations. ${ }^{174-177}$ In the following section, we highlight recent selected insights into the mechanisms of first-row TM-based heterogeneous WOCs. To render the discussion more concise, widely studied unary oxides, such as $\mathrm{CoO}_{x}$ and $\alpha-\mathrm{Fe}_{2} \mathrm{O}_{3}$ (hematite), are selected as model systems.

2.1.1. New insights into $\mathrm{CoO}_{\boldsymbol{x}}$ catalysts. Similar to the welldocumented molecular WOCs, the presence of high-valent metal sites during catalytic cycles of first-row TM-based heterogeneous catalysts has been speculated for a long time. ${ }^{178}$ Early explorations confirmed this hypothesis because (i) operando XAS measurements near the Co K-edge demonstrated that the oxidation state of Co was higher than III, ${ }^{179}$ (ii) EPR analyses immediately after electrochemical OER trapped a $\mathrm{Co}^{\mathrm{IV}}$-OxO species, ${ }^{124,180}$ and (iii) transient absorption spectroscopy (TAS) captured long-lived holes, which were assigned to the $\mathrm{Fe}^{\mathrm{IV}}$-oxo species on hematite photoanodes. ${ }^{181-185}$ However, gaining deeper knowledge is still constrained by the fact that these non-surface sensitive techniques average the information between the catalytic surface and supportive bulk for heterogeneous WOCs. Therefore, surface-specific techniques are essential to gain insights about the most exposed catalytic sites. The innovative surface interrogation scanning electrochemical microscopy (SI-SECM) developed by Bard et al. is a facile electrochemical titration technique for the quantification of surface active sites and their time-dependent behaviour. ${ }^{186,187}$ SI-SECM utilizes a substrate electrode for the pre-oxidation of metal centers to higher oxidation states, followed by a second tipping electrode, which is employed to selectively titrate the oxidized species on the surface. Accordingly, the density of active sites on the surface of CoPi nanoparticles (NPs) was 
determined to be 11 Co atoms per square nm through in situ $\mathrm{Co}^{\mathrm{II} / \mathrm{III}}$ titration. However, the resolution of this technique is limited due to the inherent switching time (order of $100 \mathrm{~ms}$ ) between two potential states, which renders the titration of reactive $\mathrm{Co}^{\mathrm{IV}}$ species less quantitative. Nevertheless, it was concluded that only a small portion of metal sites is catalytically active in heterogeneous NPs. This was also supported by numerical modelling results of the classic redox waves, which were monitored with CV studies. ${ }^{188}$ Both experimental results indicated that the vast majority of metal centers just served as structural support in the bulk of the NPs.

To derive a model for the catalytic cycle, it is necessary to probe the chemical nature of the intermediate species on the surface. Accordingly, attenuated total reflection Fourier transform infrared (ATR-FTIR) spectroscopy was successfully implemented for probing the surface peroxide intermediates on a $\mathrm{TiO}_{2}$ photoanode film. ${ }^{189}$ This technique was also established for colloidal $\mathrm{Co}_{3} \mathrm{O}_{4}$ NPs, where two reaction intermediates with different temporal behaviors were resolved. ${ }^{190}$ Using isotope labelling experiments, the first one was confirmed to be a surface superoxide species, which was specified as the fast catalytic site where two adjacent metal centers are involved. Thus, the $\mathrm{O}-\mathrm{O}$ bond formation was described as WNA at one of the $\mathrm{Co}^{\mathrm{IV}}$-oxo sites with the assistance of another $\mathrm{Co}^{\mathrm{IV}}$-oxo site as a proton acceptor (Fig. 8a). The second reaction intermediate was assigned to isolated $\mathrm{Co}^{\mathrm{IV}}$-oxo sites and found to be catalytically slow (Fig. 8b). Compared to the electronically coupled dual-site centers, these structurally segregated $\mathrm{Co}^{\mathrm{IV}}$ oxo sites had to overcome a significantly higher energy barrier for the WNA process to proceed. To understand the formation and the exceptional activity of adjacent $\mathrm{Co}^{\mathrm{IV}}$-oxo centers, DFT calculations were performed on the $\mathrm{Co}_{3} \mathrm{O}_{4}(001)$ surface. ${ }^{191}$ Surprisingly, it only took $1.42 \mathrm{meV}$ to activate the separated $\mathrm{Co}^{\mathrm{IV}}$-oxo species for their dimerization. Moreover, the kinetic barrier for the proton exchange between a $\mathrm{Co}^{\mathrm{IV}}$-oxo site and its neighboring $\mathrm{Co}^{\mathrm{III}}-\mathrm{OH}$ site was merely $0.27 \mathrm{eV}$. Therefore, these $\mathrm{Co}^{\mathrm{IV}}$-oxo species were quite mobile on the surface. In this case, when sufficient $\mathrm{Co}^{\mathrm{IV}}$-oxo species are available, the chance of their encounter and formation of $\mathrm{Co}^{\mathrm{IV}}$-oxo dimers through random walk was found to be very high. According to the calculations, the formed $\mathrm{Co}^{\mathrm{IV}}$-oxo species could further assist the following steps (deprotonation of hydroperoxide and $\mathrm{Co}^{\mathrm{II}}$ $\mathrm{H}_{2} \mathrm{O}$ species) after WNA for $\mathrm{O}-\mathrm{O}$ bond formation. This means that only the first step ( $\mathrm{Co}^{\mathrm{III}}-\mathrm{OH}$ to $\mathrm{Co}^{\mathrm{IV}}$-oxo) demands significant energy input, while the remaining steps in the catalytic cycle can proceed spontaneously if sufficient $\mathrm{Co}^{\mathrm{IV}}$-oxo species are present on the surface. For the oxygen-bridged $\left\{\mathrm{H}_{2} \mathrm{O}-\right.$ $\mathrm{Co}_{2}(\mathrm{OH})_{2}-\mathrm{OH}_{2}$ \} edge-site on $\mathrm{Co}_{3} \mathrm{O}_{4}$, it is important to note that the $\mathrm{O}-\mathrm{O}$ bond formation proceeds exclusively through the WNA pathway since in $\mathrm{H}_{2}{ }^{18} \mathrm{O}$ labeling experiments, both the unlabeled superoxide $\left({ }^{16} \mathrm{O}^{16} \mathrm{O}\right)$ FTIR band and ${ }^{32} \mathrm{O}_{2}$ product were absent.

Direct coupling of the adjacent $\mathrm{Co}^{\mathrm{IV}}$-oxo species as alternative $\mathrm{O}-\mathrm{O}$ bond formation pathway was later confirmed with other Co-based heterogeneous analogues, which featured the key $\left\{\mathrm{H}_{2} \mathrm{O}-\mathrm{Co}_{2}(\mathrm{OH})_{2}-\mathrm{OH}_{2}\right\}$ edge-sites. For example, the first turnover analysis on Co/methylenediphosphonate oxide NPs by time-resolved O-labelling isotope-ratio membrane-inlet mass spectroscopy indicated that the $\mathrm{O}-\mathrm{O}$ bond was formed predominantly through the coupling between $\mathrm{Co}^{\mathrm{IV}}-\mathrm{O}^{\bullet}$ and $\mathrm{Co}^{\mathrm{IV}}-\mathrm{OH} .{ }^{192}$ Unfortunately, it was not possible to completely exclude the contributions from the WNA pathway due to the limited temporal resolution ( $3 \mathrm{~s}$ ) of this method, where exchange of the labelled $\mathrm{O}$ could not be avoided. To gain knowledge of the $\mathrm{O}$ exchange rate at the edge-site, Nocera et al. employed an oxygen-bridged bi-cobalt molecular model and found that the exchange of the terminal $\mathrm{OH}$ or $\mathrm{OH}_{2}$ groups with syn geometry was in the order of hours. ${ }^{193}$ This extremely slow isotope exchange rate would be in principle applicable for the edge-site of heterogeneous Co-WOCs due to their structural similarities at the molecular level. Differential electrochemical

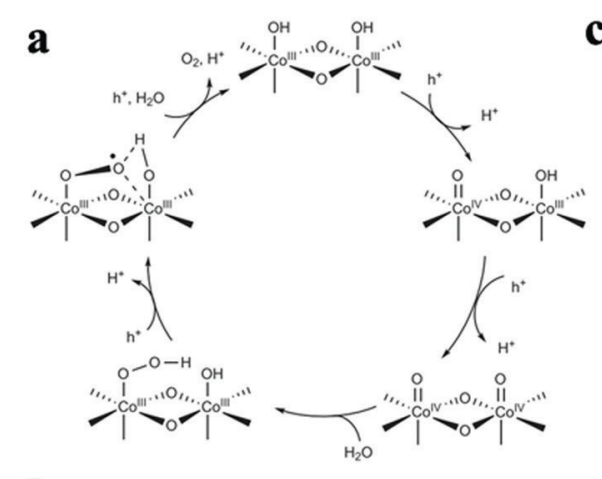

b
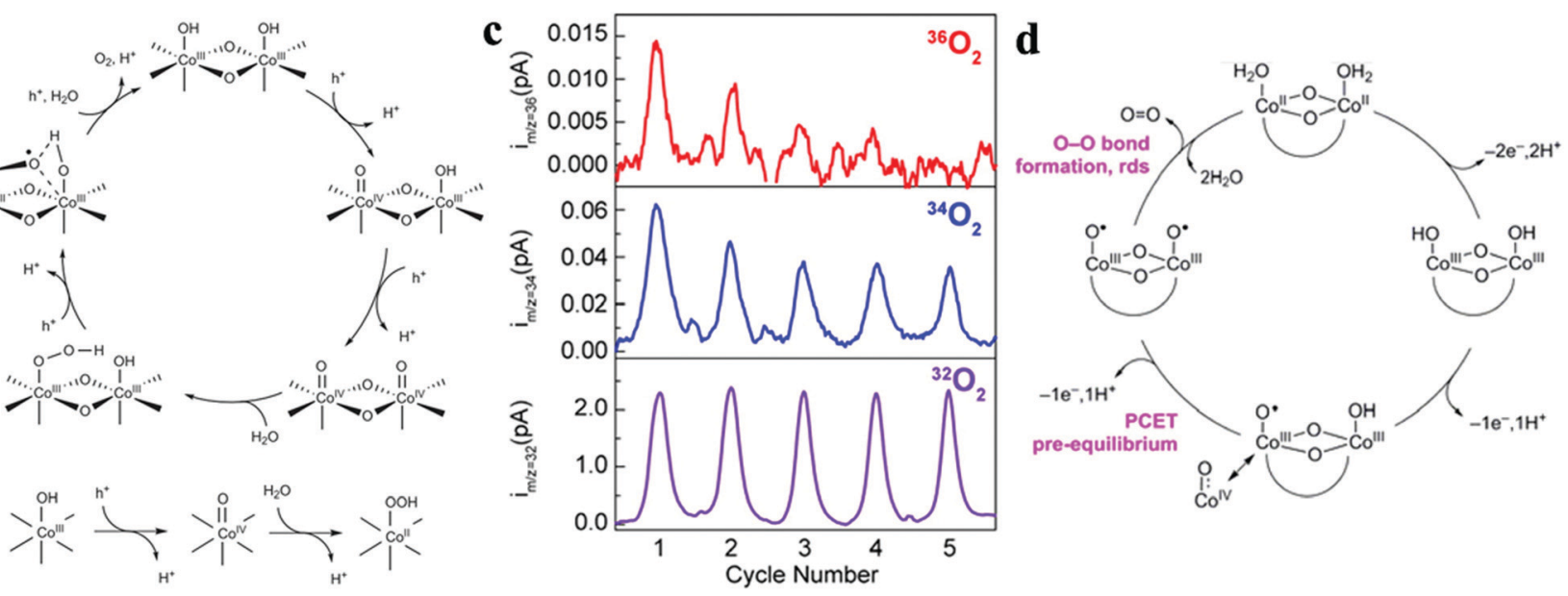

Fig. 8 Proposed reaction mechanism for the fast (a) and slow (b) catalytic sites on the $\mathrm{CO}_{3} \mathrm{O}_{4}$ surface. Reproduced with permission from ref. 190 . Copyright 2014 Springer Nature. (c) Five consecutive CVs were performed for ${ }^{18} \mathrm{O}$ labelled $\mathrm{Co}-\mathrm{WOC}\left(\mathrm{CO}_{3} \mathrm{O}_{4}\right)$ in unlabeled electrolyte, DEMS data indicate that ${ }^{36} \mathrm{O}_{2}$ deplete fast as cycle number increases. (d) Proposed reaction mechanism for the Co-WOC. Reproduced with permission from ref. 193. Copyright 2016 The American Chemical Society. 
mass spectroscopy (DEMS) experiments with ${ }^{18} \mathrm{O}$-labeled Co-WOC in unlabeled electrolyte delivered much less ${ }^{36} \mathrm{O}_{2}$ at higher cycling numbers (Fig. 8c). This indicates that the reaction proceeds primarily via the $\mathrm{Co}^{\mathrm{IV}}$-oxo coupling mechanism (Fig. 8d) and no significant structural reorganization between the terminal and bridged oxygen occurs during the reaction. Thus far, we observed that terminal hydroxide groups are generally assumed as the resting state in most catalytic cycles. However, these OER reactions were carried out under near-neutral conditions. This implies that water molecules were the actual terminal ligands, and thus water dissociation was necessary in the first step. To visualize this process, STM experiments with atomic-level resolution were carried out on $\mathrm{CoO}_{x}$ nanoislands. ${ }^{194}$ STM video monitoring revealed that water molecules first dissociated into protons and hydroxyls at the edge-sites before the basal plane took over the in following steps. The edge-site activity in water dissociation was further verified by $\mathrm{CoO}_{x}$ nano-islands with different edge to basal plane ratios. Under the same water pressure, the density of hydroxyls was reversely proportional to the radius of the nanoislands. This observation confirmed the edge activity. It was also found that the presence of additional water molecules could significantly lower the energy barrier for both water dissociation and proton diffusion, and the results were supported by DFT calculations. Overall, this study highlighted the importance of edge-sites and their higher activity for water oxidation.
To maximize the Co edge-site activity, ultra-small $2 \mathrm{~nm}$ size nanocubanes were prepared to take full advantage of the edgesite activity. ${ }^{195}$ However, the TOF of these nanocubanes $\left(0.023 \mathrm{~s}^{-1} \mathrm{Co}^{-1}\right)$ was much lower than the fast catalytic site $\left(3 \mathrm{~s}^{-1}\right)$ reported by the Frei group. ${ }^{190}$ Therefore, a WNA on single $\mathrm{Co}^{\mathrm{IV}}$-oxo sites was proposed, despite the presence of a large fraction of edge-sites. To account for this discrepancy, further research into the mechanisms for generating cooperative $\mathrm{Co}^{\mathrm{IV}}$-oxo sites is required.

We observed that the catalytic activity of the above-discussed heterogeneous Co-WOCs was primarily assigned to the $\mathrm{Co}^{\mathrm{III}}$ centers in the $\mathrm{CoO}_{6}$ octahedral geometry instead of the $\mathrm{Co}^{\mathrm{II}}$ centers in the tetrahedral geometry in the spinel structure of $\mathrm{Co}_{3} \mathrm{O}_{4}$. To distinguish between the roles of the two Co centers in different coordination environments, a cation-substitution strategy was systematically employed by Liu et al. to prepare spinel-type $\mathrm{ZnCo}_{2} \mathrm{O}_{4}$ and $\mathrm{CoAl}_{2} \mathrm{O}_{4} \cdot{ }^{196}$ With the help of operando electrochemical impedance spectroscopy (EIS) and XAS analysis, the catalytic activity (normalized current density based on the electrocatalytic active surface area) revealed that the $\mathrm{Co}^{\mathrm{II}}$ centers in tetrahedral geometry were more active than the $\mathrm{Co}^{\mathrm{III}}$ centers in the octahedral geometry (Fig. 9a). Operando Raman studies by the same group further identified the newly evolved $1068 \mathrm{~cm}^{-1}$ band as the superoxide species on the pristine $\mathrm{Co}_{3} \mathrm{O}_{4}$ surface. ${ }^{197}$ This characteristic reaction intermediate band was largely suppressed
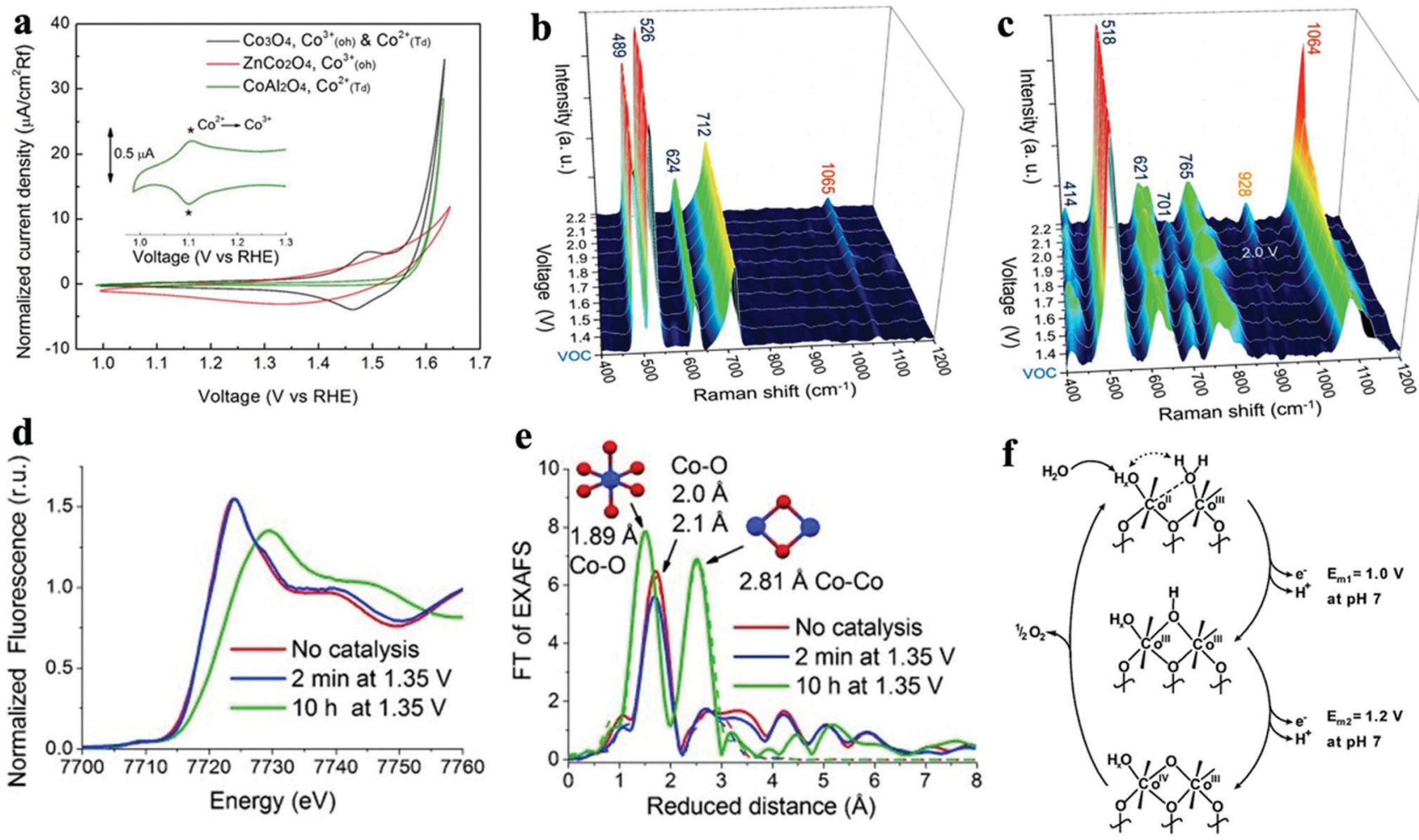

Fig. 9 (a) Normalized electrochemical activity comparison of $\mathrm{CO}_{3} \mathrm{O}_{4}, \mathrm{ZnCO}_{2} \mathrm{O}_{4}$, and $\mathrm{CoAl}_{2} \mathrm{O}_{4}$. In situ Raman spectra of (b) $\mathrm{ZnCO}_{2} \mathrm{O}_{4}$ and (c) $\mathrm{CoAl} \mathrm{O}_{4}$ as a function of applied potential. Reproduced with permission from ref. 196 and 197. Copyright 2016, The American Chemical Society. XANES (d) and Fourier-transformed EXAFS (e) spectra of crystalline CoPi before and after anodic operation (1.76 V vs. RHE) for 2 min and 10 h. Reproduced with permission from ref. 199. Copyright 2015, Wiley VCH. (f) Schematic illustration of the three structural motifs during the OER. Reproduced with permission from ref. 12. Copyright 2015, The Royal Society of Chemistry. 
for $\mathrm{ZnCo}_{2} \mathrm{O}_{4}$ (Fig. 9b), while present in $\mathrm{CoAl}_{2} \mathrm{O}_{4}$ (Fig. 9c), thus indicating the crucial role of the tetrahedrally coordinated $\mathrm{Co}^{\mathrm{II}}$ centers for the formation of this intermediate species. This observation is in contrast with previous hypotheses that octahedrally coordinated $\mathrm{Co}^{\mathrm{III}}$ edge-sites are the dominant catalytically active species. ${ }^{190,193}$ One possible explanation for these opposite conclusions is that the nature of the catalytic centers may have changed even for related starting materials considering that many previous studies have reported substantial material transformations during catalytic reactions. High-resolution transmission electron microscopy (HRTEM), for example, revealed surface reorganizations of crystalline oxides and the in situ formation of amorphous overlayers. ${ }^{9,198,199}$ Dau et al. made pioneering contributions on this key issue of the structural transformations of heterogeneous catalysts under operational conditions. ${ }^{199}$ To illustrate the reconstruction of CoPi, they utilized a variety of techniques ranging from in-house spectroscopic characterizations (UV-vis and FTIR) to synchrotron-based structural analyses (XANES in Fig. 9d and EXAFS in Fig. 9e). The restructuring of the crystalline solids was actually under kinetic control. In a representative study by Jaegermann et al., $\mathrm{CoO}_{x}$ electrocatalysts with different preparative histories exhibited distinct material transformation routes, and finally displayed very different catalytic activities. $^{200}$ This highlights the fact that electronic state differences will lead to divergent reaction pathways and apparent performance differences. Moreover, instead of the traditionally proposed surface activity that prevails for crystalline materials, the concept of volume activity was proposed for the newly formed amorphous materials. ${ }^{199}$ By applying operando XAS analyses, it was found that even amorphous Co-WOCs undergo interconversion between three different local molecular motifs (Fig. 9f). ${ }^{12}$ These molecular-scale motifs were actually found to be in dynamic equilibrium during the OER. Furthermore, a reduction of the activity by $10 \%$ was observed when ${ }^{18} \mathrm{O}$ isotope tests were performed, which served as evidence for the direct coupling of $\mathrm{Co}^{\mathrm{IV}}$-oxo species during the RDS. ${ }^{14}$

2.1.2. Hematite. Besides $\mathrm{CoO}_{x}$, hematite offers dual functionality as both a light absorber and OER catalyst, which provides the opportunity to avoid the complications inferred by the use of photosensitizers in mechanistic studies. ${ }^{201}$ Similar to other first-row TM oxides, the high-valent iron centers on hematite photoanodes have long been proposed until the first direct ATR-FTIR evidence was provided by the Hamann group. ${ }^{202}$ The $\mathrm{Fe}^{\mathrm{IV}}$-oxo species displayed characteristic absorption bands at around $898 \mathrm{~cm}^{-1}$, and the intensity of the peak was dependent on both the potential and light illumination (Fig. 10a and b, respectively). This behavior indicates that the same intermediate species were present in both the electrocatalytic and photoelectrocatalytic processes. Light illumination could provide extra photovoltage to lower the onset potential for the appearance of both reaction intermediates and catalytic current. In principle, a first-order reaction of surface holes can be proposed for the isolated $\mathrm{Fe}^{\mathrm{IV}}$-oxo sites, and WNA on these reaction intermediates will then be the RDS for $\mathrm{O}-\mathrm{O}$ bond formation. This hypothetical reaction mechanism was later supported by isotope labelling experiments and analyzing
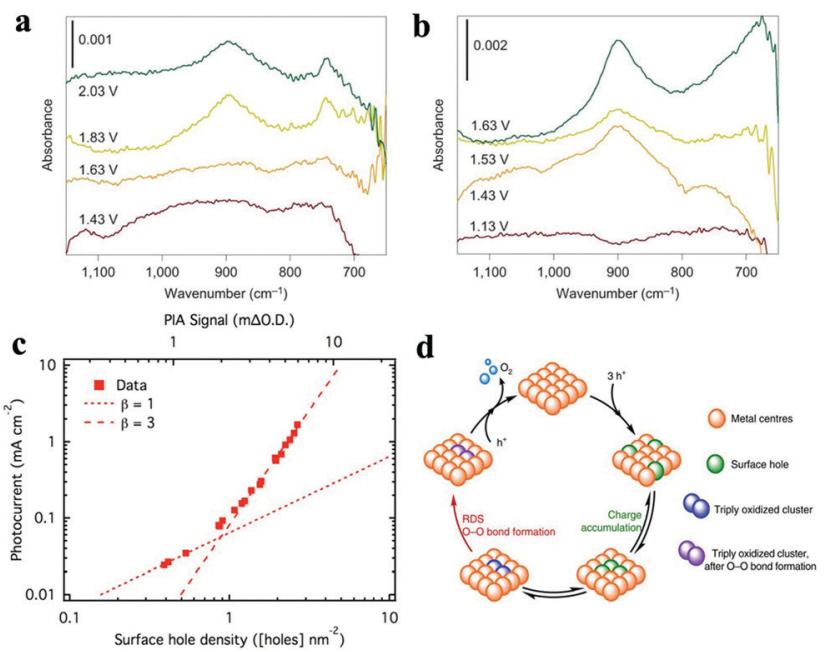

Fig. 10 Operando ATR-FTIR spectra of hematite scanned in the dark (a) and under illumination (b), with the reaction intermediate band at $898 \mathrm{~cm}^{-1}$ emerging at $1.83 \mathrm{~V}$ vs. RHE in the dark and $1.43 \mathrm{~V}$ vs. RHE under illumination. Reproduced with permission from ref. 202. Copyright 2016, Springer Nature. (c) Rate law analysis of surface hole density, where the surface hole density was derived from the intensity of the PIA signal at $650 \mathrm{~nm}$ at a steady state (1.5 V vs. RHE, $1 \mathrm{M} \mathrm{NaOH})$. Reproduced with permission from ref. 204. Copyright 2015, The American Chemical Society. (d) Proposed mechanism for the third-order reaction: the equilibrium between triply oxidized edge-sites and three surface holes is followed by the RDS for $\mathrm{O}-\mathrm{O}$ bond formation. Reproduced with permission from ref. 205. Copyright 2020, Springer Nature.

the $\mathrm{O}_{2}$ products using isotope ratio mass spectroscopy. ${ }^{203}$ With the combination of photoinduced absorption spectroscopy (PIA) and transient photocurrent spectroscopy (TPS), a first-order reaction was actually observed by Durrant et al. when the illumination intensity was weak (Fig. 10c). ${ }^{204}$ When the surface hole density surpassed a certain threshold level at higher illumination intensities, a rapid third-order reaction was observed (Fig. 10c). Recently, this high reaction order of surface holes was rationalized considering the $\mathrm{Fe}(\mathrm{OH})-\mathrm{O}-\mathrm{Fe}(\mathrm{OH})$ sites, where dynamic equilibrium was proposed between three surface holes and this edge-site dimer (Fig. 10d). ${ }^{205}$ The biphasic behavior of the reaction order as a function of hole density suggested a change in the reaction mechanism. DFT calculations indicated that the sluggish first-order reaction had an energy barrier of about $0.3 \mathrm{eV}$, while a negligible activation energy of $0.06 \mathrm{eV}$ was calculated for the third-order reaction. It is surprising that the first- and third-order reactions for the hematite photoanode match well with the 'slow' and 'fast' reaction sites observed for $\mathrm{Co}_{3} \mathrm{O}_{4}{ }^{190}$ This indicates that the cooperation of adjacent metal centers is possibly a common prerequisite for the efficient OER on metal oxide surfaces. Clearly, the accumulation of oxidation equivalents on the oxide surface is crucial for the mechanistic transition. In addition, Zhao and coworkers observed mechanistic transitions as a function of the electrolyte $\mathrm{pH}$, where slow WNA to the $\mathrm{Fe}^{\mathrm{IV}}$-oxo site was dominant at near-neutral conditions and fast adjacent oxo-coupling occurred in strongly alkaline conditions. ${ }^{206,207}$

With these and many other recent advances in mechanistic insights into unary first-row TM-based heterogeneous WOCs, 
it is clear that we are much closer to a full picture of the water oxidation cycle. The progress discussed here is inspirational and sometimes transferable for the rapidly growing number of first-row bi- or multi-metallic TM-based heterogeneous systems. It should be emphasized that the combination of many different techniques for multi-dimensional surface and structural information is required for the establishment of reliable and complete catalytic cycles. $^{23}$ For example, time-resolved FTIR can provide molecular information of the active sites in action, ${ }^{208,209}$ while operando optical and XAS spectroscopy can uncover the electronic structure of the involved metal centres. ${ }^{210}$

\subsection{Mechanistically driven design of heterogeneous WOCs}

Advanced DFT simulations are an alternative approach to access the underlying OER mechanisms of heterogeneous WOCs. Nørskov et al. first studied the OER activity of a series of oxides and proposed that the binding energies of different reaction intermediates $\left(\mathrm{HO}^{*}, \mathrm{O}^{*}\right.$, and $\left.\mathrm{HOO}^{*}\right)$ were responsible for the origin of the OER activity of the investigated catalysts. ${ }^{211}$ A universal scaling relationship between the adsorption energies of $\mathrm{HOO}^{*}$ and $\mathrm{O}^{*}$, which are generally applied for the investigation of the reaction free energy diagrams of all the oxides, was discovered. This scaling relation gives rise to an activity volcano plot as a function of specific intermediates (Fig. 11a). Moreover, it suggests that the RDS of the free energy diagram plays a vital role in the OER activity. For instance, if the oxygen binding energy is too high, the OER overpotential is
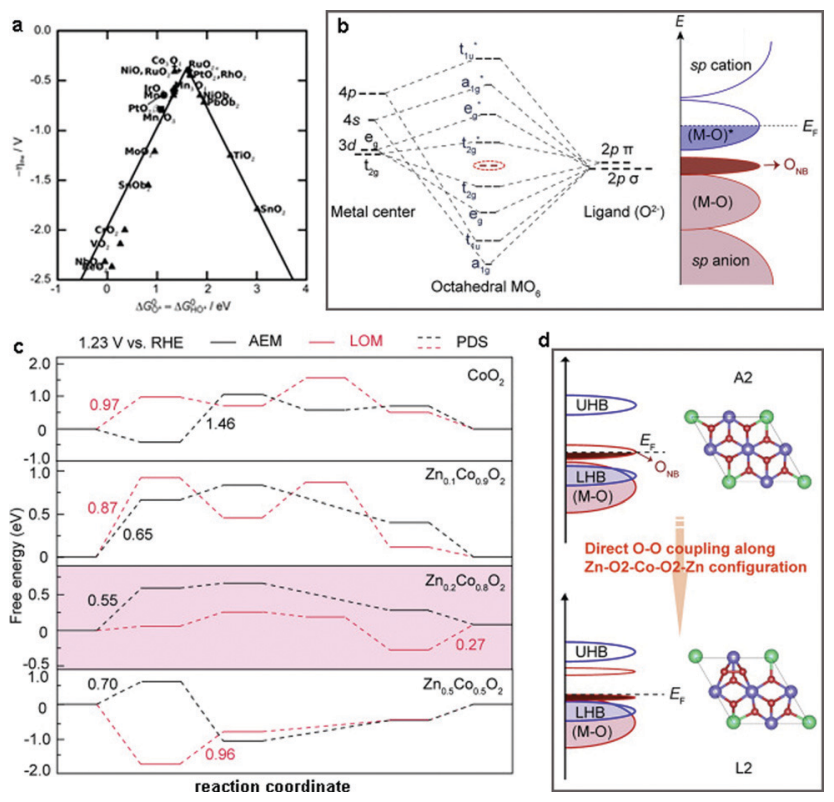

Fig. 11 (a) OER activity trends vs. standard free energy of the $\Delta G_{\mathrm{HO}^{*}}$ $\Delta G_{O^{*}}$ step. Reproduced with permission from ref. 211. Copyright 2011, Wiley $\mathrm{VCH}$. (b) Theoretical models for the formation of $\mathrm{O}_{\mathrm{NB}}$ with molecular orbital energy diagram for $\mathrm{MO}_{6}\left(E_{\mathrm{F}}=\right.$ Fermi level). (c) Calculated free energies for the OER via AEM and LOM mechanisms over $\mathrm{CoO}_{2}$ and $\mathrm{Zn}_{x} \mathrm{CO}_{1-x} \mathrm{O}_{2}$. (d) Transformation of the OER mechanism from AEM to the LOM with the absence of unoccupied oxygen states in $\mathrm{Zn}_{0.2} \mathrm{Co}_{0.8} \mathrm{O}_{2}$. Reproduced with permission from ref. 224. Copyright 2019, Springer Nature. limited by the formation of $\mathrm{HOO}^{*}$ species, otherwise, the energy barrier of $\mathrm{HO}^{*}$ species formation is dominant when the oxygen binding energy is low. Therefore, to optimize the OER activity, methods to stabilize $\mathrm{HOO}^{*}$ species compared to $\mathrm{HO}^{*}$ species are desirable, and some general strategies are discussed below.

2.2.1. Substitution strategies with other metal centers. The introduction of secondary elements with similar electron configurations into the lattice of pristine catalysts is an effective strategy to regulate their electronic structures, to redistribute the electron densities in the active metal centers, and to modulate the adsorption/desorption energies of intermediates, thereby providing the opportunity to tune the underlying OER mechanisms. ${ }^{212-214}$ For instance, binary 3d TM oxide/oxyhydroxide combinations in catalysts, such as $\mathrm{CoNi},{ }^{215} \mathrm{CoFe}^{216}$ $\mathrm{NiFe}^{217} \mathrm{CoV},{ }^{218} \mathrm{NiV},{ }^{219} \mathrm{CoMo},{ }^{220} \mathrm{NiMo}^{221} \mathrm{CoMn}^{222} \mathrm{NiMn}^{223}$ $\mathrm{CoZn},{ }^{224}$ and CoAl with a main group element, ${ }^{225}$ have been widely investigated. Among them, NiFe-based oxyhydroxides are the best-known bimetallic OER electrocatalysts. However, the question why Fe doping substantially improves the sluggish OER activity of Ni-based oxyhydroxides still remains unanswered to a large extent in heterogeneous catalyst research. With this question in mind, the effects of Fe substitution on the OER activity and mechanisms of Ni-based oxyhydroxides were investigated by Peter et $a .^{217}$ Analysis of operando XAS and voltammetric experiments first revealed a drastic influence of Fe substitution on the redox behavior of Ni centers. This phenomenon showed that the Ni-based oxyhydroxides underwent a gradual mechanism conversion when Fe was inserted. The resulting super Nernstian behavior was mainly due to the presence of a non-concerted proton-electron (PT/ET) pathway, where a negatively charge oxygen ligand is involved and can react with hydroxide ions to form '-OOH' or '-OO' intermediates. The energy barrier of this PT/ET pathway is much lower than the conventional PCET pathway.

Recently, Wang et al. reported that the OER activity and mechanism of $\mathrm{CoOOH}$ could be appropriately modulated by the incorporation of redox-inert $\mathrm{Zn}^{2+}$ ions. Combined experimental and theoretical investigations showed that the insertion of $\mathrm{Zn}^{2+}$ into $\mathrm{CoOOH}$ could promote the generation of additional oxygen non-bonding states $\left(\mathrm{O}_{\mathrm{NB}}\right)$ with different local configurations (Fig. 11b). ${ }^{224}$ The presence of $\mathrm{O}_{\mathrm{NB}}$ can stabilize the peroxoor superoxo-related $\mathrm{O}-\mathrm{O}$ intermediates, thereby regulating the OER mechanism. ${ }^{226-228}$ Furthermore, DFT calculations confirmed that the less directional $\mathrm{Zn}-\mathrm{O}$ bonds enhanced the electrical conductivities $(\sigma)$ by overlapping with the neighboring $\mathrm{O}(2 \mathrm{p})$ orbitals. As expected, the generated oxygen holes in the $\mathrm{O}_{\mathrm{NB}}$ states together with the $\mathrm{Zn}-\mathrm{O} 2-\mathrm{Co} 2-\mathrm{O} 2-\mathrm{Zn}$ configuration tuned the oxygen binding energies and further promoted the change from a conventional adsorption evolution mechanism (AEM) to a lattice oxygen oxidation mechanism (LOM) (Fig. 11c and d). Consequently, the elaborately designed electrocatalyst $\mathrm{Zn}_{0.2} \mathrm{Co}_{0.8} \mathrm{OOH}$ with rich $\mathrm{Zn}-\mathrm{O} 2-\mathrm{Co} 2-\mathrm{O} 2-\mathrm{Zn}$ configurations exhibited the best OER activity with the highest intrinsic mass activity of $0.38 \mathrm{~mA} \mathrm{~cm}_{\mathrm{ox}}{ }^{-2}\left(331.1 \mathrm{~A} \mathrm{~g}^{-1}\right)$ at $1.5 \mathrm{~V}$ vs. RHE, which was 38(61) and 19(75) times better than that of $\mathrm{CoOOH}$ and commercial $\mathrm{IrO}_{2}$, respectively. However, lower 
intrinsic activities were found in $\mathrm{Zn}_{0.3} \mathrm{Co}_{0.7} \mathrm{OOH}$ and $\mathrm{Zn}_{0.4} \mathrm{Co}_{0.6} \mathrm{OOH}$, which was mainly due to the lower content of $\mathrm{Zn}-\mathrm{O} 2-\mathrm{Co} 2-\mathrm{O} 2-\mathrm{Zn}$ motifs. Therefore, high amounts of $\mathrm{Zn}$ substitution (over 20\%) would decrease the OER activities and cause severe structural degradation of the catalysts due to the excessive amounts of oxygen vacancies. These observations were also confirmed by the investigation of the OER mechanism in spinel-type $\mathrm{ZnCo}_{2} \mathrm{O}_{4}$, which showed a decrease in OER activity compared to $\mathrm{Co}_{3} \mathrm{O}_{4}$ spinel due to the absence of $\mathrm{Zn}-\mathrm{O} 2-\mathrm{Co} 2-\mathrm{O} 2-\mathrm{Zn}$ motifs. ${ }^{196,224}$ In conclusion, we suggest the following guidelines for the selection of secondary metal centers: (i) the charge conductivity of the catalyst matrix should be increased by regulation of its electronic structure, (ii) the adsorption energies of the reaction intermediates should be optimized by proper redistribution of the electron densities, and (iii) the reaction pathways should be accelerated by alteration of the energy barrier of certain steps.

2.2.2. Improvement of heterogeneous WOCs through the introduction of vacancies. A wider range of recent studies reported that intrinsic vacancies (including cation and oxygen vacancies) in heterogeneous catalysts can tune their electronic configuration, promote the electron transfer, and generate more active sites, thereby enhancing the OER activity. ${ }^{170,212,229-236}$ Consequently, engineering oxygen vacancies $\left(\mathrm{V}_{\mathrm{O}}\right)$ has emerged as a convenient approach to improve the OER activity of first-row TM oxides or hydroxides. In a representative study, Guo et al. introduced abundant $\mathrm{V}_{\mathrm{O}}$ on the surface of hollow $\mathrm{Co}_{3} \mathrm{O}_{4}$ nanoparticles, thereby increasing the number of active sites and enhancing their catalytic performance. ${ }^{235}$ Liu et al. employed the laser ablation techniques to produce $\mathrm{V}_{\mathrm{O}}$-rich $\mathrm{Co}_{3} \mathrm{O}_{4}$ (L-CO), which exhibited enhanced electrical conductivity and high OER activity. ${ }^{236}$ The presence of oxygen vacancies was further confirmed by $\mathrm{O}$ K-edge soft XAS measurements, where a new peak appeared at around $533.4 \mathrm{eV}$, indicating the presence of $\mathrm{V}_{\mathrm{O}}$ in L-CO (Fig. 12a). To gain insight into the influence of $\mathrm{V}_{\mathrm{O}}$ on the OER mechanism, DFT simulations were carried out on L-CO and defect-free spinel $\mathrm{Co}_{3} \mathrm{O}_{4}$ (C-CO). The calculated adsorption/desorption energy scheme indicates that the free energy barriers of the OER intermediates for L-Co were considerably reduced compared to C-CO (Fig. 12b). Consistent with this prediction, L-CO exhibited the lowest overpotential of $271 \mathrm{mV}$ at a current density of $10 \mathrm{~mA} \mathrm{~cm}^{-2}$, which was $70 \mathrm{mV}$ lower than that of C-CO.

Other recent studies have further demonstrated that the introduction of metal cation vacancies $\left(V_{M}\right)$ is an effective alternative strategy to modulate the electronic structure and to tune the oxygen binding energy toward highly active OER catalysts. $^{212,234,237,238}$ For example, Zou et al. synthesized Co-deficient $\mathrm{Co}_{3-x} \mathrm{O}_{4}$ and identified it as an efficient OER electrocatalyst. $^{234}$ DFT calculations were conducted to investigate the electronic structure of $\mathrm{Co}_{3} \mathrm{O}_{4}$ before and after the introduction of $\mathrm{V}_{\mathrm{Co}}$. The simulated results showed that the formation energy of $\mathrm{V}_{\mathrm{Co}^{2+}}$ was lower than that of $\mathrm{V}_{\mathrm{Co}^{3+}}$, indicating that the preferential type of vacancies in $\mathrm{Co}_{3} \mathrm{O}_{4}$ is $\mathrm{V}_{\mathrm{Co}^{2+}}$. Moreover, the presence of high $\mathrm{V}_{\mathrm{Co}^{2+}}$ concentrations can modulate the electron density of the Co centers in $\mathrm{Co}_{3-x} \mathrm{O}_{4}$, resulting in the electronic delocalization of the neighboring atoms and further increase of the overlap of the electron wavefunctions between the $\mathrm{Co}$ and $\mathrm{O}$ atoms. Consequently, the electronic delocalization effect enhanced the charge densities around the conduction band edge and narrowed the band gap in Co-deficient $\mathrm{Co}_{3-x} \mathrm{O}_{4}$ (Fig. 12d). In fact, the insertion of $\mathrm{V}_{\mathrm{Co}^{2+}}$ is accompanied by structural distortions, which decreased the surface energy and enhanced the structural stability during the OER. Consequently, the modulated Co-deficient $\mathrm{Co}_{3-x} \mathrm{O}_{4}$ displayed remarkable OER activity with a much lower overpotential of $268 \mathrm{mV}$ at a current density of $10 \mathrm{~mA} \mathrm{~cm} \mathrm{~cm}^{-2}$ in comparison with that of normal $\mathrm{Co}_{3} \mathrm{O}_{4}$ (376 mV) (Fig. 12c). Overall, we noticed that the strategy of vacancy introduction is attracting increasing attention as an effective way to significantly improved catalyst properties, from exposing more surface sites to enhanced electrical properties. ${ }^{239}$ However, fundamental questions, such as 'the more vacancies, the better?' and 'are vacancies stationary or mobile?' remain to be answered for fully understanding the role of these structural defects.

2.2.3. Lattice strain as a tuning parameter. Tuning the lattice strain, either compressive or tensile, is a useful approach to tailor the electronic structure and to adapt the binding energies of the OER intermediates for first-row TM oxides or hydroxides. ${ }^{240-244}$ Compressive strain can result in a higher occupancy of the $\mathrm{d}_{3 z^{2}-r^{2}}$ orbitals and weaker metal-oxygen chemisorption, while tensile strain is beneficial for $\mathrm{d}_{x^{2}-y^{2}}$ orbital occupation and enhances the metal-oxygen covalency. ${ }^{242,244}$ Due to the modifications of the metal-oxygen chemisorption and covalency, the energy barriers of the OER intermediates are altered, thereby enhancing the OER activity. ${ }^{216,245-247}$ Recently, Du et al. studied the strain effects on the OER activity of epitaxial $\mathrm{NdNiO}_{3}$ thin films. ${ }^{244}$ They found that the introduction of compressive strain could indeed enhance the $\mathrm{d}_{3 z^{2}-r^{2}}$ orbital occupation, while the tensile strain was consistent with preferential occupation of the $d_{x^{2}-y^{2}}$ orbital (Fig. 12h). To investigate the strain effects on the OER activity, $\mathrm{NdNiO}_{3}$ (NNO) films were grown on different substrates with modulated strains from $-1.39 \%$ to $2.49 \%$. When $\mathrm{SrLaAlO}_{4}$ (SLAO) was employed as substrate, the resulting compressive strain of $-1.39 \%$ was the most favorable for the OER activity for NNO (Fig. 12i and j). Moreover, the compressive strain caused structural distortion of the $\mathrm{NiO}_{6}$ motifs in NNO, thereby reducing the $\mathrm{Ni}-\mathrm{O}$ bond lengths and elongating the $\mathrm{Ni}-\mathrm{O}-\mathrm{Ni}$ bonds. Furthermore, the orbital overlap or $\mathrm{p}-\mathrm{d}$ hybridization was reinforced, producing more stabilized metallic states, and thereby enhancing the OER kinetics. As expected, the hybrid NNO/SLAO showed the best OER activity among the series of investigated substrates supporting NNO films. Recently, Liu et al. prepared NiFe-LDH with tensile strain via a facile ball-milling method. ${ }^{243}$ They showed that the tensile strain could indeed improve the OER activity of NiFe-LDH (Fig. 12e). Furthermore, DFT calculations were carried out to understand the effect of tensile strain on the OER kinetics in more detail. As illustrated in Fig. 12f, the tensile strain was simulated by stretching the metal oxygen bonds by $0.16 \AA$ compared to the pristine crystal structure of NiFe-LDH. Consequently, the NiFe-LDH with tensile strain showed enhanced electron density around the Ni and Fe centers. Furthermore, the calculated free energy profiles corroborated that the theoretical OER overpotential of NiFe-LDH decreased from $0.55 \mathrm{~V}$ to $0.48 \mathrm{~V}$ 

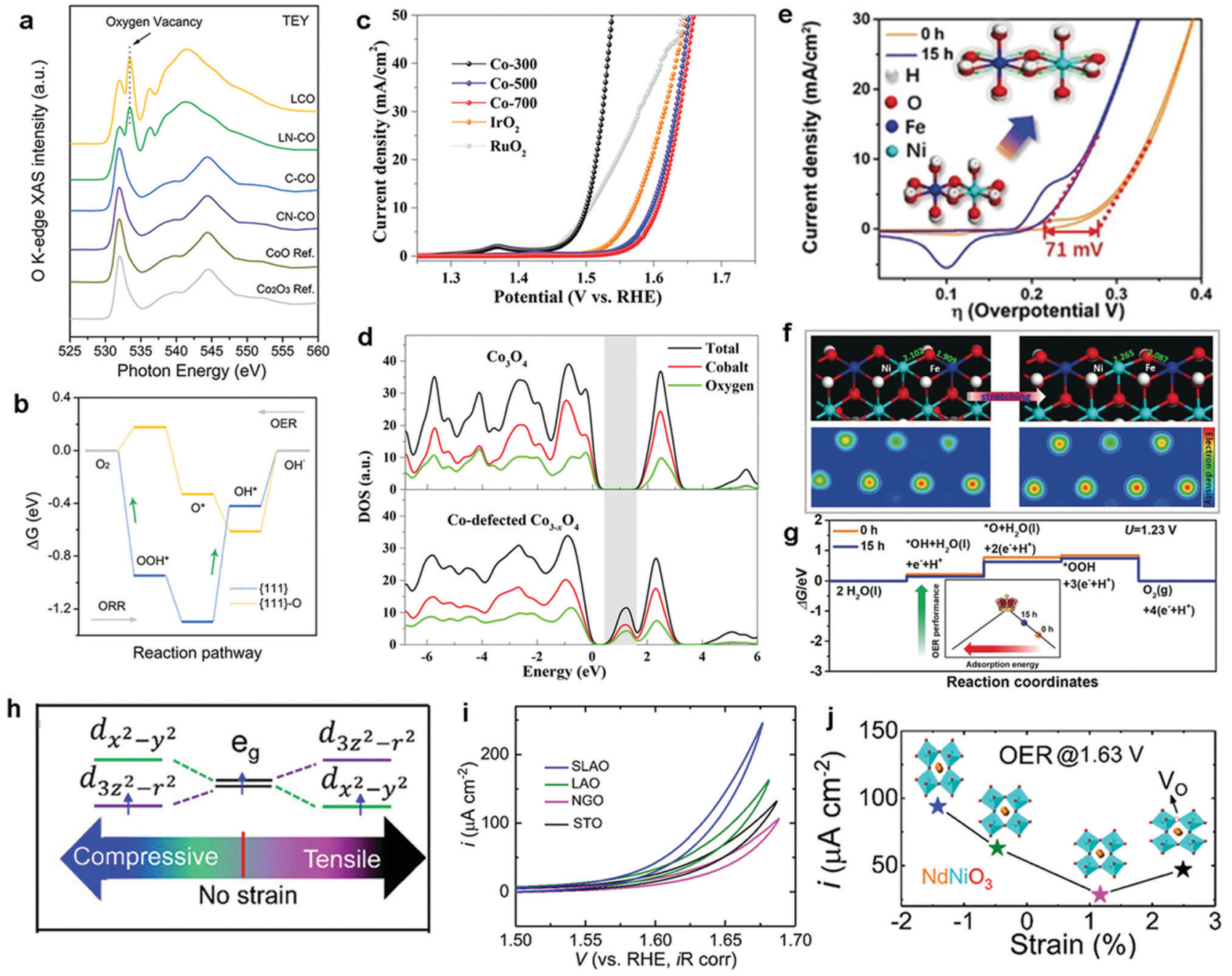

Fig. 12 (a) $\mathrm{O}$ K-edge soft XAS spectra of L-CO, $\mathrm{LN}-\mathrm{CO}, \mathrm{C}-\mathrm{CO}$, and $\mathrm{CN}-\mathrm{CO}$ and $\mathrm{CoO}$ and $\mathrm{CO}_{2} \mathrm{O}_{3}$ as references. (b) Calculated free energy profiles for the OER/ORR on $\{111\}$ planes before and after the insertion of oxygen vacancies (green arrow = RDS). Reproduced with permission from ref. 236. Copyright 2019 Wiley VCH. (c) LSV curves of three catalysts and $\mathrm{IrO}_{2}$ and $\mathrm{RuO}_{2}$ references for the OER. (d) Computational $\mathrm{DOS}$ for $\mathrm{CO}_{3} \mathrm{O}_{4}$ and $\mathrm{Co}_{-}$-deficient $\mathrm{CO}_{3-x} \mathrm{O}_{4}$. Reproduced with permission from ref. 234. Copyright 2018, The American Chemical Society. (e) OER performance of pristine NiFe-LDH and $\mathrm{NiFe}-\mathrm{LDH}$ after $15 \mathrm{~h}$ of ball-milling treatment. (f) Theoretical slab model of NiFe-LDH with and without ball-milling treatment. (g) Computed free energy profiles for (f). Reproduced with permission from ref. 243. Copyright 2019, Wiley VCH. (h) Schematic plots for the strained NNO with $e_{\mathrm{g}}$ orbital degeneracy. (i) OER performance for the NNO films grown on different substrates in $\mathrm{O}_{2}$-saturated $0.1 \mathrm{M} \mathrm{KOH}$. (j) Plots of current density recorded at $1.63 \mathrm{~V}$ of NNO films against the induced strain. Reproduced with permission from ref. 244. Copyright 2019, The American Chemical Society.

after the introduction of tensile strain into the crystal structure (Fig. 12f and g). As expected, the energy barrier of the OER intermediates was optimized, which shifted the activity of NiFeLDH towards the vertex of the activity volcano (inset in Fig. 12g). The beneficial effects of lattice strain on the electronic structures and electrocatalytic activity are also evident from other recent studies on Co-based catalysts. ${ }^{240}$ Clearly, the introduction of lattice strain leads to structural distortions and changes in bond lengths. Correspondingly, the electronic structures of the metal sites are altered, which can be selectively employed to tailor the adsorption energies of the reaction intermediates.

2.2.4. Selective exposure of crystal planes. Nanoscale crystals with exposed specific crystal planes do not only affect the geometric shape of the final products but also tune their surfacedependent physicochemical properties. ${ }^{212,248-250}$ Therefore, the rational design of catalysts exposing their most favorable active facets is a promising route towards rapid OER kinetics. ${ }^{250-253}$ For example, well-defined spinel cobalt oxides with exposed (100) and (111) planes were prepared for the investigation of facet-dependent electrocatalytic activity. ${ }^{248}$ The electrochemical measurements and theoretical studies showed that the (111) plane of $\mathrm{Co}_{3} \mathrm{O}_{4}$ exposed a higher density of cobalt ions compared to the (100) plane, thereby offering more catalytically active centers. This resulted in an enhancement in OER activity upon exposure of the (111) plane compared to that of the (100) plane. The (100) plane could stabilize the crystal structure and ensure better OER stability due to its lower surface energy compared to that of the (111) plane. Ma et al. prepared surface-tailored $\mathrm{Co}_{3} \mathrm{O}_{4}$ nanocrystals and investigated the effects of different crystal planes on their OER activity, including 
a

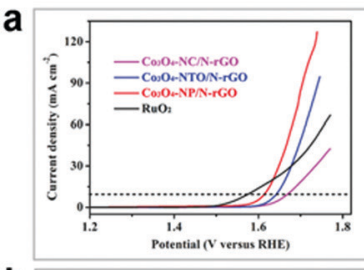

b

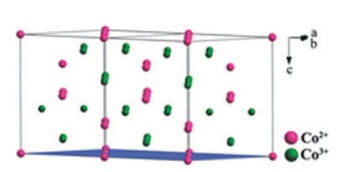

(001)

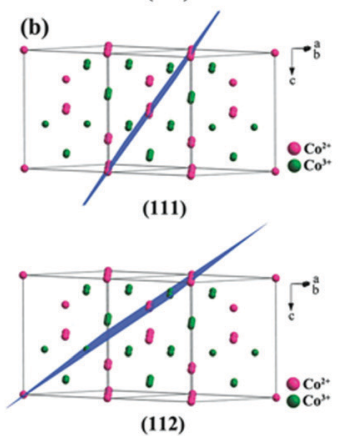

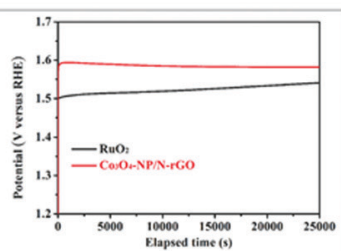
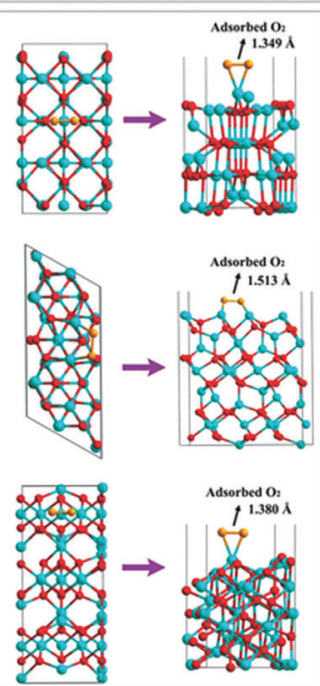

C

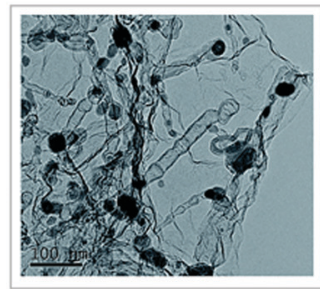

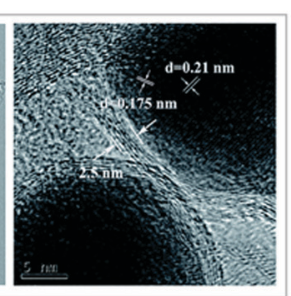

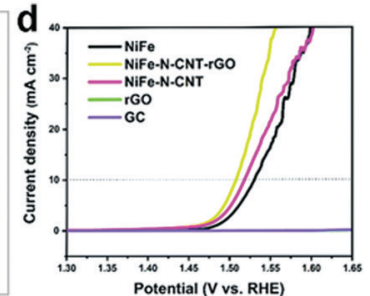

e

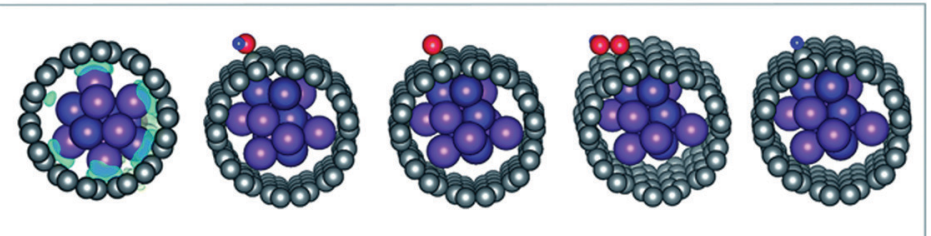

f
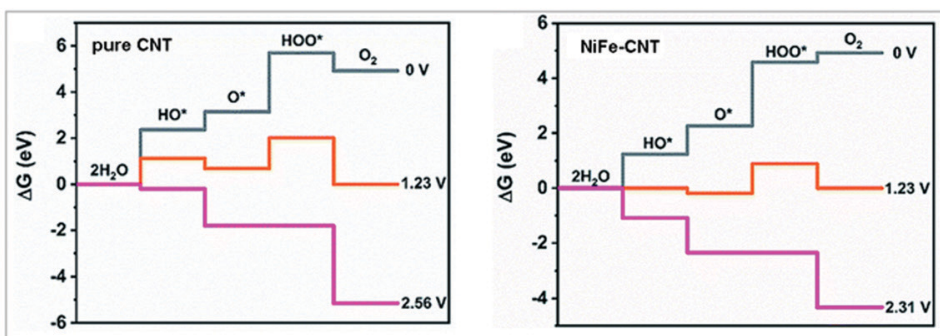

Fig. 13 (a) LSV curves for the investigated cobalt oxide OER catalysts. (b) Surface atomic configurations of $\mathrm{Co}^{2+} / \mathrm{Co}^{3+}$ on different $\mathrm{crystal}^{\mathrm{planes}}$ Reproduced with permission from ref. 251. Copyright 2018, Wiley VCH. (c) TEM and HRTEM images of NiFe-N-CNT-rGO. (d) LSV curves of as-prepared $\mathrm{NiFe}, \mathrm{NiFe}-\mathrm{N}-\mathrm{CNT}-\mathrm{rGO}, \mathrm{NiFe}-\mathrm{N}-\mathrm{CNT}$, and $\mathrm{rGO}$, with blank GC as a reference. (e) Schematic structures of OER intermediates adsorbed on NiFe-CNT. (f) Calculated free energy for OER over pure CNT and NiFe-CNT at zero potential $(U=0)$, equilibrium potential $(U=1.23 \mathrm{~V})$, and minimal potential where all steps become downhill. Reproduced with permission from ref. 171. Copyright 2019, The Royal Society of Chemistry.

nanocubes (NC) with exposed (001) planes, nanostructured octahedra (NTO) with exposed (001) and (111) planes, and nanopolyhedra (NP) with exposed (112) planes. ${ }^{251}$ The electrochemical measurements demonstrated that the $\mathrm{Co}_{3} \mathrm{O}_{4} \mathrm{NP}$ exhibited superior OER electrocatalytic activity (Fig. 13a). In terms of surface atomic configurations, the as-prepared $\mathrm{Co}_{3} \mathrm{O}_{4}$ NC, NTO, and NP nanostructures exposed different surface atomic arrangements of the $\mathrm{Co}^{2+} / \mathrm{Co}^{3+}$ active sites. The $\mathrm{Co}_{3} \mathrm{O}_{4} \mathrm{NC}$ and NTO only displayed tetrahedrally coordinated $\mathrm{Co}^{2+}\left(\mathrm{Co}^{2+} \mathrm{Td}\right)$ sites, while both the $\mathrm{Co}^{2+} \mathrm{Td}$ and octahedrally coordinated $\mathrm{Co}^{3+}\left(\mathrm{Co}^{3+}\right.$ oh $)$ sites were accessible in the $\mathrm{Co}_{3} \mathrm{O}_{4}$ NPs (Fig. 13b). DFT calculations further revealed that the rich presence of $\mathrm{Co}^{3+}$ oh sites on the (112) plane could promote the electron-donating ability and offer a moderate binding energy for the intermediates, resulting in an enhancement in the OER activity. These observations are consistent with the above discussions of studies in Section 2.1.1, where the $\mathrm{Co}^{3+}$ edge-sites were reported to be more catalytically active in well-crystalline spinel-type $\mathrm{Co}_{3} \mathrm{O}_{4}$. In addition, we want to highlight the fact that surface amorphization of crystalline $\mathrm{Co}_{3} \mathrm{O}_{4}$ NP has been demonstrated under operando conditions. ${ }^{9}$ Therefore, it is subject to further research whether this structural change of the surface may also happen for other highly crystalline oxides or not.

2.2.5. Interface engineering. Hybridizing WOCs with conductive substrates not only promotes the charge transfer, but also constructs a beneficial catalytically active interface, increases the electrocatalytically active surface area, and optimizes the adsorption/desorption energies of the OER intermediates, thereby enhancing the OER activity. ${ }^{217,226,254-256}$ The construction of carbon-encapsulated architectures has served as an alternative strategy to obtain efficient hybrid OER catalysts with high surface areas. ${ }^{171,256-259}$ The carbon matrix can protect the active species from a harsh environment and maintain the structural integrity of the catalytically active compounds. Meanwhile, the encapsulated active metal centers can also tune the electronic properties of the outer carbon shells, thereby regulating the binding energies of the OER intermediates on the carbon surface. ${ }^{171,256,257,260}$ Recently, our group developed a convenient approach for the encapsulation of metal catalysts in nitrogen-doped carbon nanotubes $(\mathrm{M}-\mathrm{N}-\mathrm{CNTs}, \mathrm{M}=\mathrm{Ni}, \mathrm{Co}, \mathrm{Fe}) .{ }^{171}$ Subsequently, coupling to reduced graphene oxide (rGO) afforded hybrid NiFe-N-CNTrGO electrocatalysts, which excelled through high specific surfaces and large fractions of exposed active sites. As shown in Fig. 13c, the TEM images clearly manifested the encapsulated core (metal)-shell (carbon) structures of NiFe-N-CNTrGO. When the as-prepared catalysts were employed as anodic materials, NiFe-N-CNT-rGO exhibited a good OER performance with an overpotential of $270 \mathrm{mV}$ at $10 \mathrm{~mA} \mathrm{~cm}$ cm $^{-2}$ (Fig. 13d). Moreover, NiFe-N-CNT-rGO possessed a higher BET surface area of $160 \mathrm{~m}^{2} \mathrm{~g}^{-1}$ compared with that of NiFe 
alloy $\left(30 \mathrm{~m}^{2} \mathrm{~g}^{-1}\right)$, further indicating that the encapsulated carbon shell can not only protect the catalytically active metal center from inevitable surface reconstruction processes, but also provides a high surface area for the catalytic reactions. To gain insight into the relation between interface engineering and the OER kinetics, DFT calculations were carried out for the as-prepared catalyst. As presented in Fig. 13e, the electron densities of the $\mathrm{Ni}$ and $\mathrm{Fe}$ atoms were redistributed after encapsulation into the CNTs. Since the surface charge states of the metal ions play an important role in the surface-anion adsorption, their redistribution is expected to influence the binding energy of the OER intermediates. Indeed, the calculated theoretical overpotential of NiFe-CNT (1.08 V) was significantly reduced compared with that of bare NiFe $(2.64 \mathrm{~V})$ and CNT (1.33 V) (Fig. 13f).

Recently, Khani et al. reported a convenient two-step sol-gel and carbothermal reduction strategy and prepared a series of graphitic-shell encapsulated metal catalysts (denoted as G-Ni, G-Co, G-Fe, G- $\mathrm{Ni}_{0.5} \mathrm{Fe}_{0.5}, \quad \mathrm{G}-\mathrm{Ni}_{0.5} \mathrm{Co}_{0.5}, \mathrm{G}-\mathrm{Co}_{0.5} \mathrm{Fe}_{0.5}$, and $\left.\mathrm{G}-\mathrm{Ni}_{1 / 3} \mathrm{Co}_{1 / 3} \mathrm{Fe}_{1 / 3}\right) .{ }^{256}$ Various ex situ techniques including powder XRD, Raman, and XPS spectroscopy confirmed that some metallic nanoparticles without the protection of graphitic shells were converted into metal oxide species after the OER. The graphitic-shell encapsulation of the metal catalysts not only preserved their pristine crystal structure, but also maintained their high electrocatalytic activity for a long time under the harsh electrochemical conditions. The results showed that the as-prepared $\mathrm{G}-\mathrm{Ni}_{0.5} \mathrm{Fe}_{0.5}$ showed a low overpotential of $280 \mathrm{mV}$ at $10 \mathrm{~mA} \mathrm{~cm}^{-2}$. All the above studies demonstrate that the rational utilization of a protective matrix material is a promising strategy for maintaining the structural integrity and high OER activity of electrocatalysts. It should be noted that many electrocatalysts generally underwent surface structural reconstruction under harsh oxidative conditions, which could affect their electrocatalytically active species and surface areas. Since this makes mechanistic investigations more complex, researchers should focus on the exploration of the real active species and aim to understand the dynamic processes under the operational reaction conditions. These challenges for the investigation of heterogeneous WOCs are summarized in the next sections.

\subsection{Challenges in the mechanistic investigation of heterogeneous WOCs}

The influence of many different parameters on the water oxidation performance, such as catalyst surface change, redox chemistry, defect chemistry, electrolyte, gas-liquid-solid interface, and adsorption/desorption energy as a function of the mentioned parameters, render a deep understanding of the catalytic process challenging. Therefore, the assignment of a distinct catalytic mechanism to a given heterogeneous water oxidation catalyst requires considerable analytical efforts to address all the issues discussed in the following sections.

2.3.1. Surface amorphization. Considering that surface amorphization remains a severe issue, exploring the true active catalyst under the operational reaction conditions is absolutely indispensable for deriving a precise reaction mechanism. Specifically, OER catalysts often undergo structural reconstruction during the electrochemical oxidation process, which further contributes to remarkable changes in the TOF. ${ }^{261}$ In this case, the change in the electrochemically active surface area (ECSA) should be considered for a fair comparison. In other cases, the degradation of nanoparticles under the OER conditions turned out to be the limiting factor for the system stability and performance. ${ }^{262}$ This phenomenon considerably changed the traditional perception of the true catalyst in solidstate catalysis.

Perovskites have attracted particular interest among heterogeneous catalysts recently. ${ }^{263-267}$ Advances in this field have shown that the apparently stable perovskites can indeed transform into other phases when exposed to the corrosive conditions of water splitting. ${ }^{268-272}$ Suntivich et al. showed that in the initial stages of the catalysis, the crystalline $\mathrm{Ba}_{0.5} \mathrm{Sr}_{0.5} \mathrm{Co}_{0.8^{-}}$ $\mathrm{Fe}_{0.2} \mathrm{O}_{3-\delta}$ mixed oxide (BSCF) changes and forms an amorphous mixed oxide shell on the surface, which acts as the true catalyst in the OER (Fig. 14a-d). ${ }^{273}$ In the operando electrochemical XAS study by Schmidt et al., the structural reconstruction and the dynamic surface amorphization of BSCF were resolved in detail. ${ }^{274}$ In this case, the Co atoms reached higher oxidation states and acted as the major catalytic centers in the BSCF structure.

Additionally, in situ FT-EXAFS spectra further clarified that the growth of an amorphous $\mathrm{Co} / \mathrm{FeO}(\mathrm{OH})$ shell on the electrodeelectrolyte interface was induced by the lattice OER process (Fig. 14e and f). ${ }^{274}$ Surface reconstruction of these mixed oxides is usually accompanied by changes in the distribution of shortrange order, the formation of local defects in the lattice structure, and oxygen vacancy changes. ${ }^{275,276}$ All these key factors must be considered in fundamental investigations of the OER mechanism.

The influence of surface corrosion on the lattice counteranions makes the mechanistic understanding of heterogeneous OER catalysts even more complicated, giving rise to in-depth investigations. ${ }^{277,278}$ Despite the considerable OER activity of non-oxide first-row TM compounds, such as sulfides, selenides, and nitrides, these compounds are not thermodynamically stable under the highly oxidative OER conditions and are mostly converted to metal oxide hydroxide phases. Recently, various characterizations have been performed to screen the surface evolution and phase transition of non-oxide compounds during their OER performance. ${ }^{277-280}$ Fan et al. applied in situ HRTEM to study the structural changes of an amorphous $\operatorname{CoS}_{x}$ chalcogenide during the OER. Their study showed that in the first step, the $\operatorname{CoS}_{x}$ pre-catalyst is transformed into a $\mathrm{Co}(\mathrm{OH})_{2}$ intermediate. Next, the intermediate undergoes irreversible conversion into a crystalline $\mathrm{CoOOH}$ phase on the surface. ${ }^{279}$

In selected cases, the transformation of the crystalline precatalyst into an amorphous phase as the true active catalyst has been identified as a reversible process with different in situ X-ray techniques. $^{9,281}$ The possibility of these reversible phenomena makes the precise identification of catalytically active sites via operando methods for the mechanistic understanding of OER highly important. 

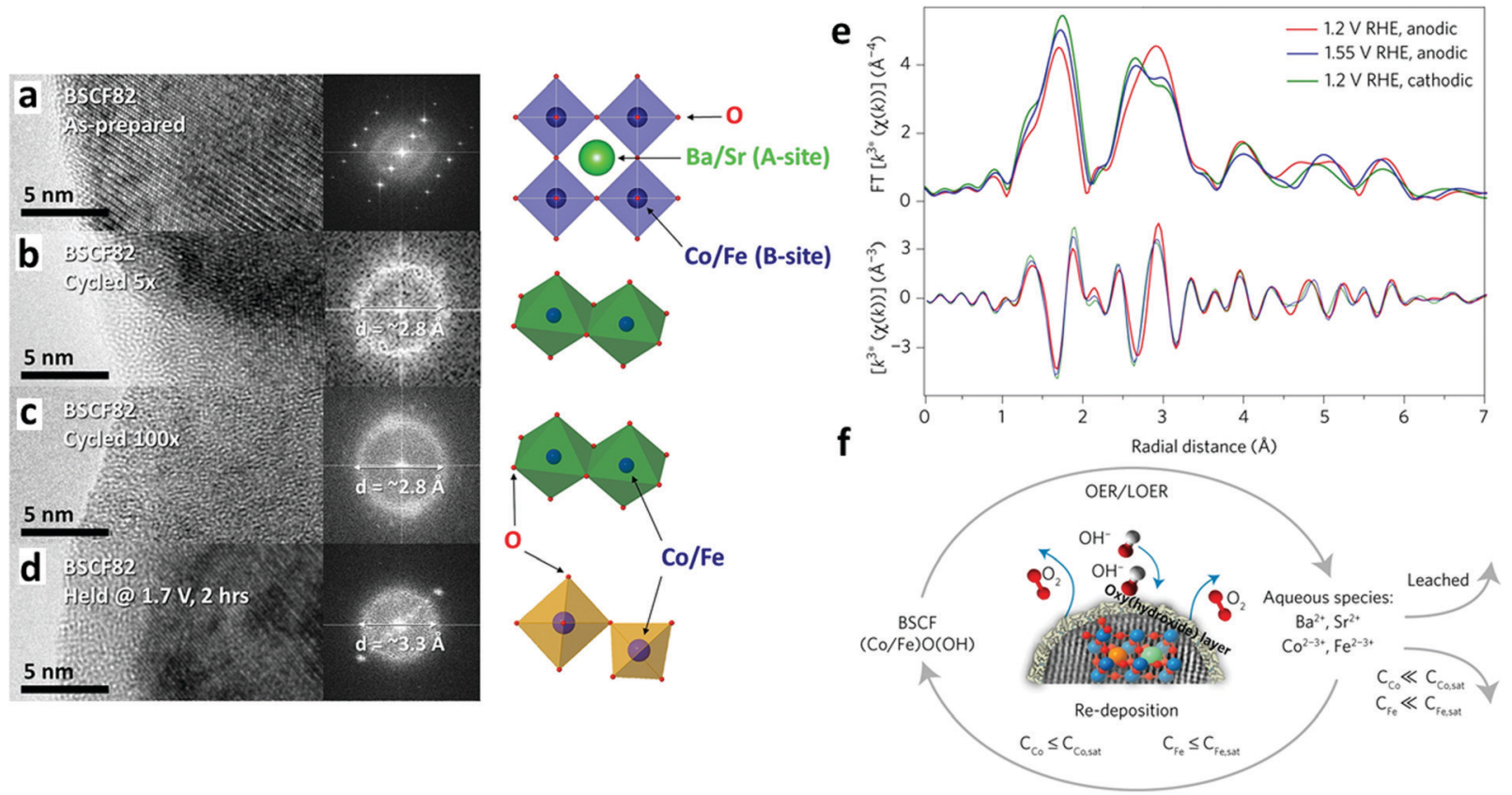

Fig. 14 HRTEM images and corresponding fast Fourier transforms (FFT) of (a) pre-catalytic $\mathrm{Ba}_{0.5} \mathrm{Sr}_{0.5} \mathrm{Co}_{0.8} \mathrm{Fe}_{0.2} \mathrm{O}_{3-\delta}$ (BSCF82) perovskite and BSCF82 samples after (b) 5 cycles and (c) 100 cycles. (d) Post-catalytic BSCF82 sample after $2 \mathrm{~h}$ chronopotentiometry at $1.7 \mathrm{~V}$ vs. RHE in $0.1 \mathrm{M} \mathrm{KOH}$ electrolyte at $10 \mathrm{mV} \mathrm{s}^{-1}$. Reproduced with permission from ref. 273. Copyright 2012, The American Chemical Society. (e) Fourier transform (FT) $k^{3}$-weighted Co K-edge EXAFS spectra of BSCF-FS sample at different applied potentials in $0.1 \mathrm{M} \mathrm{KOH}$. The shoulder at $\sim 3.5 \AA$ and the peak at $\sim 4 \AA$ correspond to Co-Ba/Sr and $\mathrm{Co}-\mathrm{Co} / \mathrm{Fe}$ in corner-sharing octahedra in the perovskite structure, respectively. (f) Schematic representation of amorphization and lattice OER processes in BSCF-FS, which leads to leaching of $\mathrm{Ba}^{2+}$ and $\mathrm{Sr}^{2+}$ cations and dissolution of $\mathrm{Co}$ and Fe cations, respectively. Reproduced with permission from ref. 274. Copyright 2017, Springer Nature.

When trying to assess the various performance-related factors of solid-state WOCs, such as the degree of crystallinity, surface corrosion, particle size and morphology, the thickness of the electrocatalyst, and oxidation state of the active sites, the combination of different in situ methods can provide further fundamental understanding of the catalytic performance and the reaction mechanism. Recently, several in situ experiments have been undertaken to monitor the reaction intermediates and to specify redox state and local atomic structure of the active sites. Among the different applied in situ techniques, XAS, ${ }^{282-285} \mathrm{TEM}^{279,286}$ Raman, $^{287}$ and Mössbauer spectroscopy ${ }^{175,288}$ are referred to here.

2.3.2. Controversial roles of different catalytic centers. Providing precise atomic-level information about catalytically active sites in multi-metal solid-state catalysts becomes more challenging through the fast conversion of the reaction intermediates. Hunter et al. applied nonaqueous media with limited amounts of substrate $\left(\mathrm{H}_{2} \mathrm{O}\right.$ and $\left.\mathrm{HO}^{-}\right)$to trap the intermediate in NiFe-LDH-catalyzed electrochemical water oxidation. ${ }^{288}$ The authors showed that a five-coordinate cis-dioxo-Fe(vi) species is involved in the OER. ${ }^{288}$ However, Friebel et al. probed tetrahedrally coordinated iron sites together with octahedrally coordinated $\mathrm{Fe}^{3+}$ species as the active centers in electrochemical water oxidation with $(\mathrm{Ni}, \mathrm{Fe}) \mathrm{OOH} .{ }^{282}$ The authors suggested that the iron sites are the superior catalytic centers of water oxidation, while Ni was not considered as a directly active catalytic center. ${ }^{282}$ A recent operando XAS study of hyperfine $\beta$ - $\mathrm{FeOOH}$ nanorods, which were surface modified with amorphous Ni hydroxide, revealed no changes in the structural environment and valence of $\mathrm{Fe}^{3+}$ centers under water oxidation reaction conditions. ${ }^{283}$ In contrast, reversible oxidation of $\mathrm{Ni}^{2+}$ to $\mathrm{Ni}^{3+}$ occurred during electrocatalysis. ${ }^{283}$ Moreover, in another study, di- $\mu$-oxo $\mathrm{Ni}^{\mathrm{IV}}-\mathrm{Fe}^{\mathrm{III}}$ species were revealed as the active site in $\mathrm{Fe}_{100-y} \mathrm{Ni}_{y} \mathrm{O}_{x^{-}}$ catalyzed water electrolysis, while iron ions remained redox inactive. ${ }^{284}$

Impurities from the electrolyte are another crucial factor that can affect the OER activity of heterogeneous catalysts and the real active species. ${ }^{287,289,290}$ Bell et al. reinvestigated the role of Fe impurities in the OER activity of $\mathrm{Ni}(\mathrm{OH})_{2} / \mathrm{NiOOH}$. They showed that aging an Ni-(oxy)hydroxide film in unpurified $\mathrm{KOH}$ electrolyte with Fe traces led to the formation of an Fe-doped Ni-oxy/hydroxide species. The newly formed phase displayed OER activity comparable to optimized $\mathrm{Ni}_{1-x} \mathrm{Fe}_{x} \mathrm{OOH}$ catalysts. ${ }^{287}$ Najafpour et al. revealed that the presence of $\mathrm{Fe}$ impurities in $\mathrm{KOH}$ electrolyte plays a significant role in the OER activity of NiV-LDH. Using operando UV-vis spectrophotometry and EXAFS techniques, it was shown that during water oxidation in impure $\mathrm{KOH}$, the NiV-LDH catalyst is converted to a partially oxidized $\alpha-\mathrm{Ni}(\mathrm{OH})_{2} / \mathrm{NiOOH}$ phase with Fe traces and this structure acts as the true catalyst. ${ }^{290}$

Regarding the dependence of different reports on the applied techniques and respective conditions, the obtained results should be interpreted carefully. Consequently, the results of case studies should not be considered as general concepts for reliably 
predicting key performance parameters and active species. Additionally, considering the critical role of the topmost layers of the electrocatalysts in the catalytic performance of the material and the kinetics and mechanism of the reaction, comprehensive monitoring of heterogeneous catalysts with atomic level operando techniques becomes mandatory. Although many reports add information on the bulk characteristics of catalysts, information based on in situ spectroscopic methods at the atomic scale, such as environmental TEM, are now required to derive optimization concepts for solid-state WOCs. Furthermore, these data can be supported by atomic-level computational modelling and simulation techniques on the path to predictive design.

Accordingly, surface reconstruction in the gas-liquid-solid interface merits more in-depth investigations with suitable operando techniques. This approach holds the key to a more comprehensive understanding of true water oxidation catalysts. Despite the rich body of literature on true catalyst studies in water oxidation, there is an obvious and increasing need for systematic studies of catalytic interfaces at the atomic scale by operando methods. Furthermore, this is an incentive to make them more accessible for everyday lab practice and to combine the results with advanced computational methods. Accordingly, artificial intelligence and different types of machine learning can speed up the exploration of reaction intermediates and the knowledge-based discovery of stable and highly efficient WOCs. ${ }^{291-293}$

\section{Part III: perspectives for knowledge transfer between molecular and heterogeneous WOCs}

\subsection{Lessons learned from heterogeneous WOCs}

Over the past decades, progress in heterogeneous WOCs was driven by both mechanistic understanding and new catalyst design concepts, as outlined in Part II of this review. We proceed with new options to transfer this rich knowledge to the field of molecular WOC development. In the following, we present some case studies and highlight key overlap between both research areas.

3.1.1. Active molecular motifs in heterogeneous WOCs. Conventionally, it is assumed that the catalytic centers in heterogeneous compounds are most accessible on the surface of crystalline NPs, where maximum exposure to the reaction medium takes place. ${ }^{188}$ This implies that only a very small fraction of metal sites are catalytically active, while the majority just serves as structural support in the bulk. ${ }^{186}$ However, this may not apply for heterogeneous WOCs, which are mostly operated in harsh oxidative conditions, since the OER often occurs after the accumulation of a certain amount of oxidative equivalents and at high anodic potentials $(>1.23 \mathrm{~V} v s$. RHE). ${ }^{294-296}$ This renders a closer pre- and post-catalytic structural examination of the crystal surface necessary for a valid interpretation of the recorded catalytic activity. As discussed in Section 2.1.1, Dau and coworkers monitored the minor

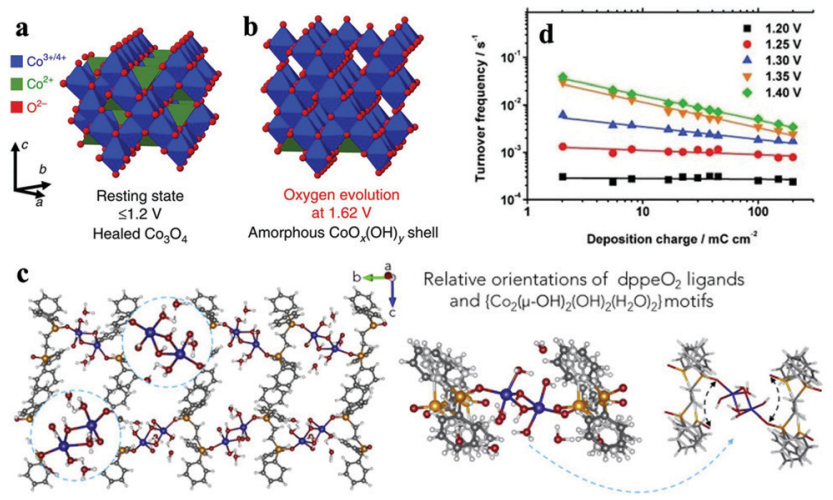

Fig. 15 Surface structure of $\mathrm{CO}_{3} \mathrm{O}_{4}$ electrocatalysts at non-catalytic (a, crystalline) and OER potentials (b, amorphous surface shell). Reproduced with permission from ref. 9. Copyright 2015 Springer Nature. (c) Molecular structure of the disordered moieties of $\mathrm{Co}-\mathrm{dppeO} \mathrm{O}_{2}$ and the distorted edge-site motif $\left\{\mathrm{H}_{2} \mathrm{O}-\mathrm{CO}_{2}(\mathrm{OH})_{2}-\mathrm{OH}_{2}\right\}$. Reproduced with permission from ref. 22. Copyright 2019, Elsevier. (d) TOF evolution as a function of different amounts of cobalt centers $\left(\mathrm{CoO}_{x}\right)$, where the nearzero slopes at lower operational potentials indicate that all the cobalt sites contribute equally across the deposited film. Reproduced with permission from ref. 304. Copyright 2014, Wiley VCH.

structural transformation on the surface of crystalline $\mathrm{Co}_{3} \mathrm{O}_{4}$ films when the applied potential was increased from the noncatalytic region $(\leq 1.2 \mathrm{~V} v s$. RHE) to the level for efficient OER (1.62 V vs. RHE). This transformation was correlated with the conversion of tetrahedrally coordinated $\mathrm{Co}^{\mathrm{II}}$ centers (Fig. 15a) into octahedrally coordinated $\mathrm{Co}^{\mathrm{III} / \mathrm{IV}}$ centers (Fig. 15b). ${ }^{9}$ The newly generated amorphous material featured a three-dimensional cross-linked di- $\mu$-oxo-bridged Co edge-site motif, which has been frequently isolated in molecular WOCs as the catalytic active site, as mentioned for example in Section 1.2.3. ${ }^{26,93,126}$ Interestingly, this molecule-related surface motif could undergo re-crystallization when the applied potential was swept back to non-catalytic values. Therefore, $\mathrm{Co}_{3} \mathrm{O}_{4}$ electrocatalysts display remarkable structural flexibility between crystalline heterogeneous materials and amorphous oxides with structural similarities to molecules.

However, this re-crystallization phenomenon is not a common observation for most electrocatalysts, which frequently display an ultrafine nano-structure or relatively low crystallinity. ${ }^{297-299}$ In this case, the 'surface only' model of catalytic heterogeneous WOCs requires adjustments since the amorphization can propagate towards the bulk region. ${ }^{199}$ The extension and rate of this irreversible structural reconstruction depends on many parameters such as the preparative history and nanostructure of heterogeneous WOCs, and on relevant reaction conditions, such as the anodic potential. Correspondingly, to derive convincing structure-activity relations for heterogeneous WOCs, confirmation of the molecular structure of newly developed amorphous materials is of great importance. ${ }^{12}$ In this regard, we employed the new disordered 1D coordination polymer WOC Co-dppeO ${ }_{2}$ as a model system and identified the edge-site $\left\{\mathrm{H}_{2} \mathrm{O}-\mathrm{Co}_{2}(\mathrm{OH})_{2}-\mathrm{OH}_{2}\right\}$ motif as the active center, which was embedded into a ligand matrix (see Section 1.1.1), in close analogy to molecular cubane 
WOCs and $\mathrm{Co}_{3} \mathrm{O}_{4} \cdot{ }^{22,26,210}$ The atomically resolved molecular motif within the highly disordered material was further refined with the coupling of in situ spectroscopic data and computational modeling (Fig. 15c, cf. Section 1.1.1). In addition, the direct utilization of amorphous WOCs promotes the participation of a higher fraction of metal centers in the catalytic reaction. ${ }^{300-303}$ For example, in a representative study, amorphous $\mathrm{CoO}_{x}$ was found to be composed of nanoscale fragments, which were interconnected by edge-sharing $\mathrm{CoO}_{6}$ octahedral dimers as a molecular motif. ${ }^{304,305}$ These dimers were randomly organized into polymeric networks. When exposed to electrolyte, they were saturated with water molecules and cations and anions from the reaction medium. This semi-permeability enabled the equal contribution of exposed metal centers when the mass diffusion was sufficient (Fig. 15d). An increasing number of studies have revealed that the real active motifs of most heterogeneous WOCs indeed bear close resemblance to molecular moieties, which is an prerequisite for mutual knowledge transfer. ${ }^{12}$

3.1.2. The $\mu$-oxo-bridged edge-site motif. As pointed out above, the edge-site $\left\{\mathrm{H}_{2} \mathrm{O}-\mathrm{M}_{2}(\mathrm{OH})_{2}-\mathrm{OH}_{2}\right\}$ motif is an important structural feature of heterogeneous WOCs. This $\mu$-oxo-bridged surface motif also represents the core component of bi- and multi-nuclear molecular WOCs to enable the cooperation of neighboring metal centers. ${ }^{4,8,306,307}$ The activation of this edgesite dimer was observed on heterogeneous WOC surfaces once a certain density of oxidative equivalents was available and these surface species had sufficient mobility. ${ }^{191}$ When both conditions are fulfilled, the edge-site motif shows high TOF for water oxidation since the $\mathrm{O}-\mathrm{O}$ bond is formed either through coupling of neighboring oxo species or via WNA of one metaloxo species with the assistance of the adjacent one. Both pathways exhibit low energy barriers. ${ }^{308}$ Experimentally, fast third-order reactions and superoxide intermediates have been determined for various metal oxide surfaces such as $\mathrm{Co}_{3} \mathrm{O}_{4}$, $\mathrm{TiO}_{2}$ and hematite. ${ }^{190,204,205}$ We are aware that this dimeric motif is not a mandatory requirement for the OER since there are many catalytically active mononuclear WOCs, as discussed in Section 1.2.1. ${ }^{11}$ However, the structurally isolated single metal centers generally exhibit lower TOFs since only one electron is transferred prior to $\mathrm{O}-\mathrm{O}$ bond formation. ${ }^{118}$ Correspondingly, slow first-order reactions and metal-oxo intermediates were detected in these cases, where the WNA to this single metal-oxo species was the RDS. ${ }^{202,210}$ Therefore, the key characteristic edge-site motif of heterogeneous WOCs can be used to design $\mu$-oxo-bridged bi- or multi-nuclear molecular WOCs. Actually, Nature's selection of its photosynthetic manganese complex is a very resilient implementation of this edge-site feature. ${ }^{309,310}$ It is widely speculated that the $\mu$-oxo-bridge can help to level off the accumulated redox equivalents and manage protons that are released during the reaction. ${ }^{311}$ To directly investigate the aspects of bio-inspiration and edge-site functionality, our group first reported a functional molecular WOC $\left[\mathrm{Co}_{4}^{\mathrm{II}}(\mathrm{dpy}\{\mathrm{OH}\} \mathrm{O})_{4}(\mathrm{OAc})_{2}\left(\mathrm{H}_{2} \mathrm{O}\right)_{2}\right]\left(\mathrm{ClO}_{4}\right)_{2} \quad\left(\mathrm{Co}_{4}^{\mathrm{II}} \mathrm{O}_{4}-\mathrm{dpk} ; \quad \mathrm{dpk}=\right.$ di(2-pyridyl)ketone) as a tunable model system with a ligand environment (Fig. 16a, $c f$. also Section 1.2.3). ${ }^{26}$ After the establishment of molecular integrity during the OER, $\mathrm{Co}_{4}^{\mathrm{II}} \mathrm{O}_{4}-\mathrm{dpk}$
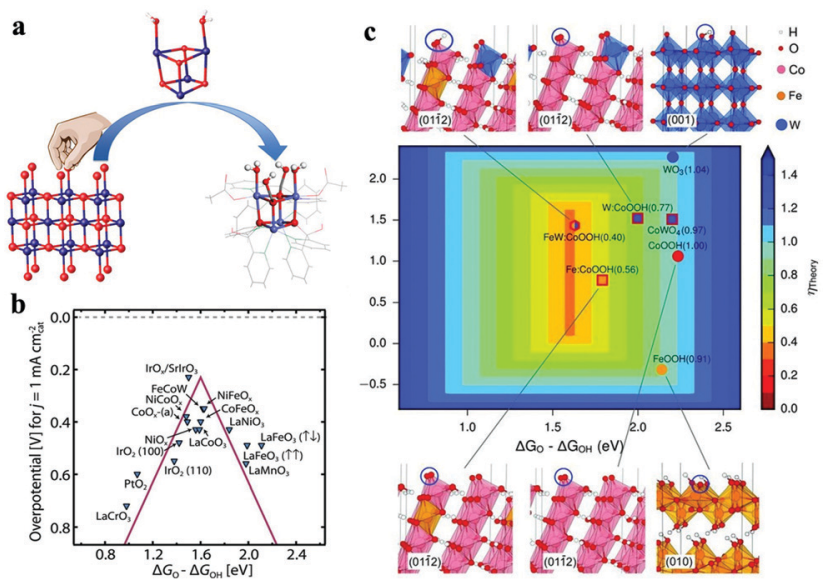

Fig. 16 (a) Structure of $\mathrm{CO}_{4}^{\prime \prime} \mathrm{O}_{4}$-dpk cubane representing a surface cut-out of the $\mu$-oxo-bridged $\mathrm{CoO}_{x}$ catalyst. Reproduced with permission from ref. 26. Copyright 2017, The American Chemical Society. (b) OER volcano plot summarized for a wide range of metal oxide surfaces employing $\Delta G_{\mathrm{O}}-\Delta G_{\mathrm{OH}}$ as a single descriptor. (c) Comparison of the theoretical OER overpotentials on pure $\mathrm{WO}_{3}(001)$, $\mathrm{COOOH}(0112)$, $\mathrm{FeOOH}(010)$ and $\mathrm{Fe}, \mathrm{W}$-doped $\mathrm{CoOOH}$, where the $\mathrm{FeCoW}$ oxyhydroxide shows the lowest overpotential. Reproduced with permission from ref. 315 and 324. Copyright 2017 and 2016, AAAS.

exhibited a higher normalized TOF per Co center than its mononuclear $\left[\mathrm{Co}^{\mathrm{III}}(\mathrm{dyp}\{\mathrm{OH}\} \mathrm{O})_{2}\right] \mathrm{ClO}_{4}$ analogue, which was protected by the same ligand environment but lacked the edge-site feature. ${ }^{312}$ Presumably, the bioinspired tetranuclear $\mathrm{Co}_{4}^{\mathrm{II}} \mathrm{O}_{4}$-dpk outperformed this compound through its edge-site structure, where proton exchange between neighboring metal centers was facilitated. In addition, the tetranuclear $\mathrm{Co}_{4} \mathrm{O}_{4}$ cubane topology had been proposed as a crucial factor for initiating the OER. ${ }^{313}$ In follow-up studies, we then brought forward a new family of cobalt cubanes with tunable reactive sites as a function of the counteranion environment, revealing the unexplored power of apparent spectator anions to customize and tune the desired structural features of targeted molecular WOCs. ${ }^{314}$

Meanwhile, it is noteworthy that Mn cubanes behave less straightforward as molecular WOCs (see Section 1.2.3). ${ }^{122}$ Along the lines of cooperative molecular nuclearity motifs, an innovative pentanuclear iron complex (shown in Fig. 5) was designed and found to exhibit a high TOF of $1900 \mathrm{~s}^{-1}$, which outperformed previous lower nuclear iron-based molecular WOCs by at least three orders of magnitude. ${ }^{135}$ The superior activity was ascribed to (i) the presence of adjacent metal centers that significantly decreased the thermodynamic barrier for intramolecular $\mathrm{O}-\mathrm{O}$ bond formation, ${ }^{99,101}$ and (ii) the large redox flexibility of the pentanuclear core, which could accommodate multiple oxidation equivalents. Therefore, we believe that the edge-site feature of heterogeneous WOCs can inspire future molecular design in the long term thanks to its remarkable flexibility in many aspects such as ligand and metal selection, along with tunability of electronic and protonation states. In addition, the efficient delocalization of oxidation equivalents over the polymeric metal centers on heterogeneous WOCs can be applied as a reciprocal 
guideline for designing molecular systems with higher nuclearity. ${ }^{118}$

3.1.3. Transferrable scaling relationships. In the heterogeneous WOC community, scaling relationships have been successfully applied for predicting the electrocatalytic performance. Thus, many experimentally determined overpotentials can well be fitted into theoretically derived $1 \mathrm{D}$ volcano plots (Fig. 16b). ${ }^{315}$ However, this concept is far less applied on molecular systems. ${ }^{316,317}$ The scaling relationships are based on a series of correlations, for example, the adsorption energies of both $\mathrm{HO}^{*}$ and $\mathrm{HOO}^{*}$ display linear character with respect to that of $\mathrm{O}^{*} .^{318,319}$ This linear correlation is beneficial for breaking down multivariable problems into one dimension, and thus the single descriptor of $\mathrm{OH}^{*}$ binding energy can be used to predict the OER rate in a classic volcano plot. ${ }^{320}$ Meanwhile, this scaling relationship indicates a defined limitation, where the minimum possible overpotential for the OER is around $0.3 \mathrm{~V}^{321}$ To overcome the inherent limitation of scaling relationships, the design of multi-component catalysts is a common strategy, where different metal sites can mediate different intermediates. In this regard, many bi- or trimetallic heterogeneous oxides have been designed to regulate the $3 \mathrm{~d}$ electronic structure of first-row TMs. ${ }^{219,322,323}$ For example, with the help of theoretical calculations (Fig. 16c), an atomically dispersed FeCoW oxyhydroxide electrocatalyst was constructed, which exhibited a record low overpotential of $191 \mathrm{mV}$ for $10 \mathrm{~mA} \mathrm{~cm}{ }^{-2}$ in alkaline conditions. ${ }^{324}$ Similarly, this strategy could be transferred to molecular systems by employing different types of catalytic centers. The cooperation of different types of intramolecular metal sites could possibly decouple different intermediate species that are constrained by the scaling relationships. Inspired by this hypothesis, we designed the first mixed $3 \mathrm{~d}-3 \mathrm{~d} \mathrm{Co} / \mathrm{Ni}$ cubane WOCs as a pioneering test system. ${ }^{26}$ Surprisingly, the mixed cubanes showed lower OER activity compared to the pristine Co-based cubanes. This contradicts the observed trends for the productive interaction of $\mathrm{Co}$ and $\mathrm{Ni}$ in bimetallic heterogeneous WOCs. ${ }^{215}$ These phenomena suggest that the replacement of the metal center in a molecular system may induce a more significant electronic effect than in their heterogeneous counterparts, where the electronic effect of the second metal would be leveled out by the bulk of interconnected metal centers. ${ }^{325}$ Nevertheless, the mixed cubane concept opens up transfer options from heterogeneous WOCs to future molecular designs. We anticipate that a moderate extent of substitution of metal centers will be beneficial for molecular systems. ${ }^{326}$ For example, Nature's PSII employs one redox inactive Ca site to fine tune the $3 \mathrm{~d}$ electronic structure of the $\mathrm{Mn}$ cubane core. ${ }^{327}$ To apply the scaling relationships on molecular WOCs, some other strategies such as utilizing redox-active ligands or tuning the topology of the metal core can be applied to regulate the electronic structure of the catalytic sites. ${ }^{328}$

Overall, the fast-growing field of heterogeneous WOCs has created many design principles and will continue to introduce more in the near future. With the discovery of shared mechanistic principles, the knowledge transfer from the pool of heterogeneous to molecular WOCs will greatly promote the design of the latter. Meanwhile, progress in molecular design can be inspiration for heterogeneous catalyst optimization, as we point out in the next section.

\subsection{Lessons from molecular studies for heterogeneous WOC design}

According to the mechanistic analyses presented in Part I and II, it is clear that molecular WOCs excel mainly through welldefined atomic structures, which is essential for an in-depth understanding of reaction intermediates. However, their isolation for heterogeneous WOCs remains a significant challenge, despite select success on some oxide surfaces such as $\mathrm{CoO}_{x}$ and $\mathrm{Fe}_{2} \mathrm{O}_{3}$ ( $c f$. Section 2.1). Therefore, identifying the structural similarities between molecular and heterogeneous WOCs at the atomic level can facilitate the transfer of mechanistic understanding between both catalyst types. In principle, this knowledge transfer can start by translating salient structural features of molecular WOCs to the surface or edge-sites of their heterogeneous counterparts. To date, the number of these examples in the heterogeneous field is still too limited to elaborate widely here. Accordingly, here we focus on the frontier research area of single-atom catalysts (SACs). SACs are presently the closest to the emerging bridging concept between molecular and heterogeneous WOCs because they feature the atomic dispersion of metal atoms on or in specific substrates as robust active centers for catalytic reactions. ${ }^{329}$ Therefore, SACs are actively connecting heterogeneous and molecular design concepts, and they are ideal platforms for bilateral knowledge transfer. In the following, we summarize recent research trends on SACs, with special emphasis on their molecular aspects of synthesis and structural-performance relationships, and on the debate on single $v s$. dual/multi-site systems.

3.2.1. Translation of molecular design principles into SACs. It is widely accepted that one of the major challenges of SAC design is the chemical stabilization of the separate metal atoms in the support. As discussed in Section 1.2, the metal centers in molecular WOCs are chemically coordinated by specific ligands, which not only keep the metal ions in place but also regulate their electronic structure. ${ }^{330}$ Therefore, the selection of suitable ligands to optimize both their structure and catalytic performance is a universal strategy for molecular design. Accordingly, defect/vacancy confinement, spatial regulation and coordination design with specific supports are widely employed in the fabrication of SACs, i.e. the supports are usually considered as the 'ligands' in SACs. ${ }^{16}$ Two types of SACs can be differentiated with respect to their interactions between metal atoms and support, i.e. support-coordinated and support-stabilized types. In the former type, the atoms from the support material are usually replaced by metal centers to form an integrated structure. The latter type is based on the stabilization of well-coordinated molecules by the support. In this type, ligands from the original molecules are well preserved, and the metal atoms are further partially coordinated by the support. In the studies of SACs, the debate on the contribution of the support materials to the catalytic activity raises the 
following key questions: where does the activity mainly arise from, the metal center, ligands or both? What exactly distinguishes support-coordinated SACs from traditional heteroatom-doped heterogeneous catalysts? The following discussion will focus on the synthesis of these two types of SACs and on addressing the above questions.

Support-coordinated SACs. Many materials have been reported as supports for SACs. Two types of supports are classified with respect to their contributions to the OER, namely non-catalytic supports and catalytic supports. In the case of non-catalytic supports, they adopt the role of "ligands", which stabilize the metal centers and regulate their charge distribution. To implant single-metal atoms into the supports, their pretreatment by inducing defects or vacancies is necessary. One of the typical examples is the immobilization of single atoms in the lattice of defective graphene oxide (GO). Yao et al. reported on a Ni-SAC, ${ }^{331}$ where they first created increasingly defective GO sheets through thermally annealing a mixture of graphene and melamine under an inert atmosphere, and then anchored Ni ions onto the surface of this pretreated GO through an electrostatic adsorption process in solution. The final Ni SACs coordinated on GO were obtained via a pyrolysis process, leading to the formation of $\mathrm{Ni}-\mathrm{C}$ bonds in the GO sheets.
In addition to the direct carbon coordination, most graphenebased SACs were coordinated by $\mathrm{N}$ atoms to form the typical $\mathrm{M}-\mathrm{N}_{4}$ moieties on the graphene lattice. For instance, Huang et al. obtained Ni-, Co- and Fe-SACs coordinated by $\mathrm{M}-\mathrm{N}_{4}$ moieties by subjecting defective graphene to a pyrolysis reaction under an $\mathrm{NH}_{3}$ atmosphere (Fig. 17a). ${ }^{332}$ The coordination geometries and the dispersion of single atoms were directly detected by HRTEM, as shown in Fig. 17b-d. N-coordination can not only modify the electronic structure of the isolated metal atoms, but also improve the adsorption of the reactants. ${ }^{333}$ In a recent study on Mn-SACs $\left(\mathrm{Mn}-\mathrm{N}_{4}-\mathrm{G}\right)$, Li et al. evidenced that the four-fold $\mathrm{N}$-coordination favors the formation of nucleophilic $\mathrm{Mn}^{\mathrm{IV}}$-oxo species during the OER, which leads to further attack of another water molecule in the electrolyte for $\mathrm{O}-\mathrm{O}$ bond formation. ${ }^{334} \mathrm{How}^{-}$ ever, the reaction mechanism in this study was solely supported by DFT calculations, and thus further experimental studies on the identification of the real functionality of $\mathrm{N}$ atoms are still expected. Other carbon-based SACs, such as those supported on CNT or $\mathrm{C}_{3} \mathrm{~N}_{4}$, were also reported as efficient WOCs emerging from similar fabrication processes. ${ }^{335,336}$

Recently, alloys were introduced as alternative supports for SACs. Li et al. reported single $\mathrm{Ru}$ atoms supported by a $\mathrm{PtCu}$ alloy for the OER, with a minimized overpotential of $90 \mathrm{mV}$ at a current density of $10 \mathrm{~mA} \mathrm{~cm}{ }^{-2}$ (Fig. 17e and f). ${ }^{337}$ They found
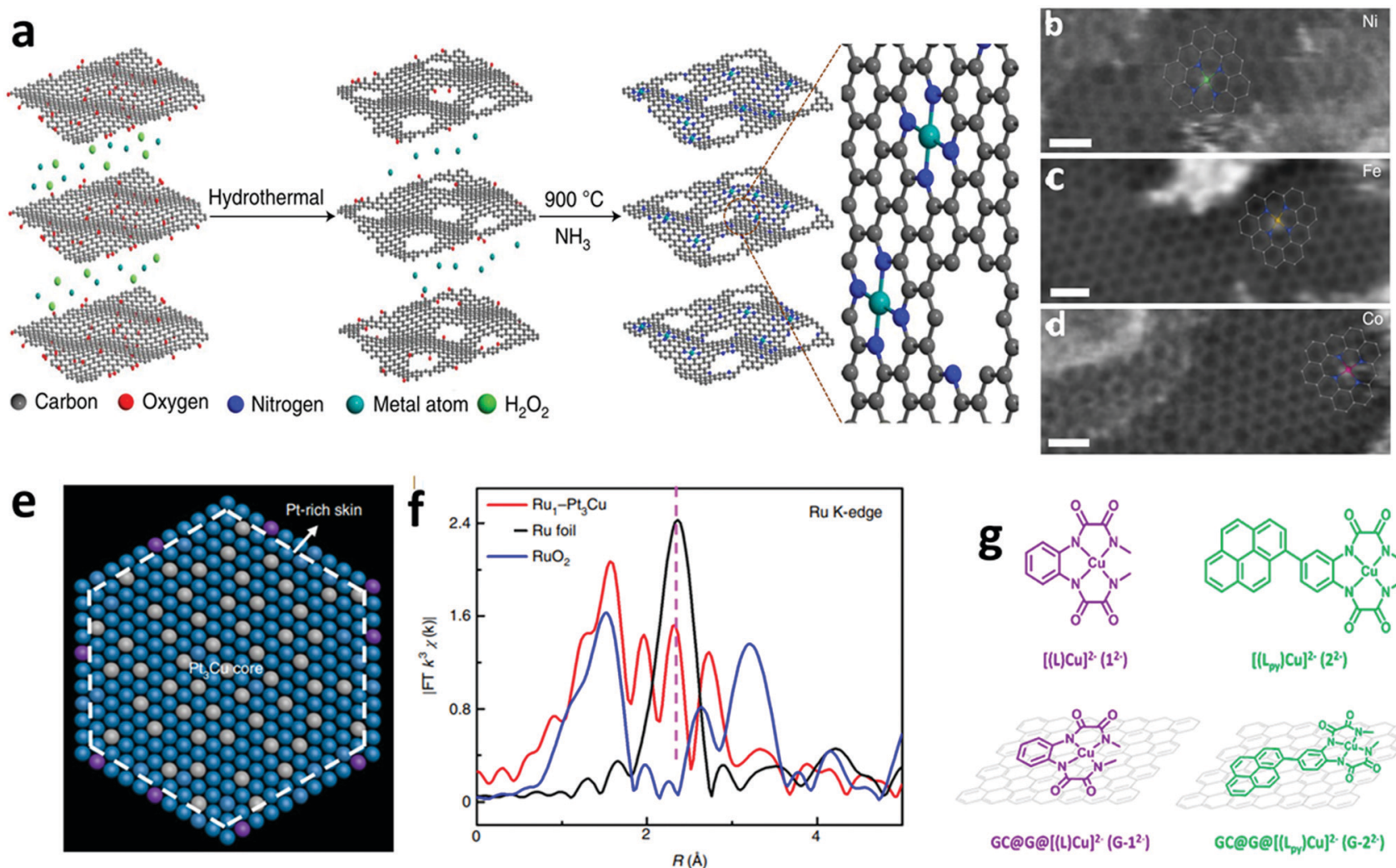

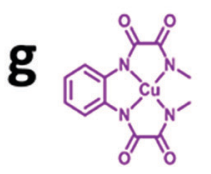

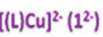
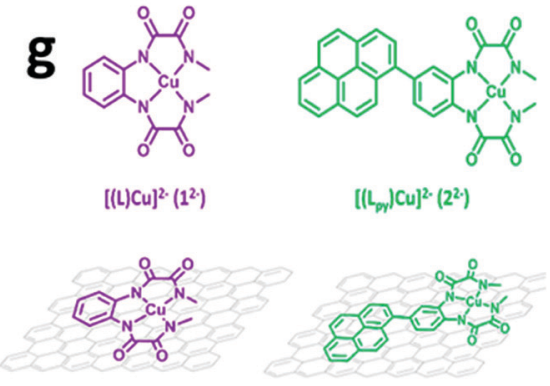

GC@G@[(L)Cu $]^{2 \cdot}\left(G-1^{2} \cdot\right)$

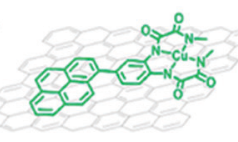

GC@G@I(L $\left.\left.L_{\text {pr }}\right) C u\right]^{2 \cdot} \cdot\left(G-2^{2 \cdot}\right)$

Fig. 17 (a) Typical synthetic protocol of SACs coordinated by defective N-doped graphene. (b-d) High-resolution TEM images of graphenecoordinated Ni, Co and Fe SACs; scale bars $=0.5 \mathrm{~nm}$. (e) Structural model showing Ru single atoms on PtCu alloy (blue, grey and purple represent $\mathrm{Pt}, \mathrm{Cu}$ and Ru, respectively). (f) FT-EXAFS spectra of the Ru K-edge and reference samples. Reproduced with permission from ref. 332 and 337 . Copyright 2018 and 2019, Springer Nature. (g) Cu molecules stabilized by Gr. Reproduced with permission from ref. 160. Copyright 2017, The American Chemical Society. 
that the compressive strain from the PtCu lattice engineers the electronic structure of the isolated $\mathrm{Ru}$ atoms through the chemical bonds formed with $\mathrm{Pt}$ and $\mathrm{Cu}$ atoms in the support. Further investigations showed that the bulk alloy could also act as an electron reservoir to donate electrons to reaction intermediates, resulting in the prevention of the over-oxidation and dissolution of Ru atoms, which enhances their stability towards the OER. ${ }^{337}$ Although non-catalytic supports do not directly participate in the OER process, they can influence the properties of single atoms by modifying the electronic structures and optimizing the adsorption energies of the reactants.

Complementing the non-catalytic supports, catalytic supports have been also widely reported in water oxidation. ${ }^{338}$ Studies on an Au/NiFe-LDH SAC system reported by Zhang et al. demonstrated that the hybridization of $\mathrm{Au}$ atoms after coordinating with $\mathrm{O}$ atoms in NiFe-LDH promoted the charge redistribution of the active $\mathrm{Fe}$ and $\mathrm{Ni}$ atoms, resulting in enhanced OER activity of the Fe sites. ${ }^{339}$ A similar result was reported by Lin et al. for Pt/NiO-SAC, where the Pt single atoms promote the phase transformation from $\mathrm{NiO}$ to $\gamma$-NiOOH rather than acting as active sites. ${ }^{340}$ On the other hand, there are also many examples of active single atoms coordinated with catalytic supports. In a representative study, Sun et al. found that the $\mathrm{Ru}$ atoms in $\mathrm{Ru} / \mathrm{CoFe}-\mathrm{LDH}$ systems are more likely to represent the active sites compared to the Fe sites. However, the authors did not state whether Co and Fe also participate as active sites in the OER. ${ }^{341} \mathrm{~A}$ similar conclusion was also reported by Ma et al. for a $\mathrm{W} / \alpha-\mathrm{Ni}(\mathrm{OH})_{2} \mathrm{SAC}^{342}$ However, in the more recently developed Ir/NiO SAC system, Gu et al. found that the single Ir atoms not only serve as the active sites for the OER but also improve the reactivity of NiO. Therein, the formed ${ }^{*} \mathrm{OH}$ species on Ni sites could easily relocate to the surrounding Ir sites to create a joint reaction pathway. ${ }^{343}$ The discussion of the real active site in a catalytic support should consider both the electronic structure effects and multi-site reaction mechanisms. Current studies mainly rely on DFT calculations, and thus more experimental evidence based on recent advanced techniques, such as ATR-FTIR and operando XAS, as mentioned in Parts I and II, is required to unambiguously identify the active sites and their reaction mechanisms in future investigations.

The discussed examples clearly show that SACs provide a platform for understanding the behavior and functionalities of isolated reaction centers and their surrounding atoms during the OER. Therefore, the design and synthesis of SACs coordinated by non-catalytic supports (such as graphene) is one of the most convenient ways to exclude the influence of the support. However, their current OER activities do not yet meet application-oriented standards. SACs coordinated by catalytic supports are much more promising in terms of their performance; however, the nature of their catalytic supports, such as oxides, alloys and hydroxides and LDHs, brings us back to the above question: what are the conceptual differences between these SACs and the traditional heterogeneous catalysts? Therefore, when investigating SACs coordinated by catalytic supports, more emphasis should be placed on understanding their coordination-performance relationships, which can bring forward future design guidelines for more active SACs in applied OER.

Support-stabilized SACs. Although the development of supportcoordinated SACs has turned into an important branch, the precise control of the coordination environment with the current widely used pyrolysis strategy for anchoring single metal atoms is still a massive preparative challenge. Researchers with expertise in molecular catalysis developed a molecule-support stabilization strategy, which paves another way to achieve both recyclable and well-coordinated SACs. However, in this protocol, establishing a strong chemical connection between molecules and solid supports remains difficult. Thus, many oxide supports have been reported to address this issue ${ }^{31}$ based on the hypothesis that molecules can be stabilized by the hydroxyl termination of the oxides, which connects most of these studies to oxide-based photochemical water oxidation using molecules as cocatalysts. ${ }^{4}$ We implemented an independent part in Section 3.3.1 to discuss this issue. Therefore, the focus in this section is on inert supports for stabilizing molecular catalysts.

Classic examples are graphene-like supports. Llobet et al. proposed a $\pi$-stacking strategy to stabilize $\mathrm{Cu}$ molecules on graphene sheets. It has been shown that the pyrene group in the molecule acts as the anchoring unit connecting the molecules to the graphene surface via $\pi$-conjugation (Fig. 17g). ${ }^{160}$ The OER performance was notably improved due to the extended $\pi$-delocalization arising from graphene. In a related study, N-doped defective graphene was used for supporting cobalt phthalocyanine (CoPc) molecules. ${ }^{344}$ The investigations showed that the Co center in the molecule was further coordinated by another $\mathrm{N}$ atom from the graphene support to form a Co- $\mathrm{N}_{5}$ moiety, acting as the active site for the OER. In addition to graphene, other carbon-based supports such as CNTs have been employed to stabilize Ru-based molecular catalysts. ${ }^{345,346}$ There are two reasons why carbon-based supports are widely developed to bridge homogeneous and heterogeneous catalysis. Firstly, their large surface area provides abundant anchoring sites to stabilize molecules; secondly, C-C $\mathrm{sp}^{2}$ hybridization provides extended $\pi$-delocalization, which offers the possibility of forming chemical bonds and electron transfer tunnels. ${ }^{347,348}$ The support-stabilized strategy can preserve the coordination environment of the original molecules to a large extent, thereby providing ample opportunities for the chemical design and optimization of molecules with high OER activities.

\subsubsection{Coordination-performance relationships of SACs. As} outlined in Part I, the design of ligands plays an important role to regulate the stability and reactivity of molecular catalysts. ${ }^{33}$ In general, amido/imine ligands with nitrogen donor(s) and/or alkoxide ligands with oxygen donor(s) are employed for molecular WOCs because they can stabilize the high oxidation states of metal centers during catalytic redox cycles. ${ }^{349}$ Therefore, the majority of current SACs are coordinated by four nitrogen atoms. As stated above, SACs can be considered as 'heterogenized molecular catalysts', where the support takes over the ligand function. Therefore, adjustment of the coordination environment in SACs can enhance their OER performance. ${ }^{350}$ Huang et al., for example, 
found that the $\mathrm{C}$ atoms connected to $\mathrm{N}$ atoms in the second coordination shell of support-coordinated $\mathrm{NiN}_{4} \mathrm{C}_{4}$ SAC are also preferential binding sites for the OER due to the doping-induced charge redistribution around the $\mathrm{M}-\mathrm{N}_{4}$ moiety. ${ }^{332}$ The authors found that the participation of $\mathrm{C}$ atoms in the OER process depends strongly on the number of d electrons $\left(N_{\mathrm{d}}\right)$ of the metal in $\mathrm{MN}_{4} \mathrm{C}_{4}$ moieties ( $\mathrm{M}=$ metal atoms). Similar conclusions were also made for Ir-based SACs stabilized by an NiO support, where the Ir atoms were stabilized by covalent bonding to $\mathrm{O}$ atoms and were considered as auxiliary active sites in the OER process. ${ }^{343}$ To systematically investigate the role of the coordination environment, Zeng and Du et al. designed different coordination environments for the most widely studied first-row TMs in the graphene lattice and theoretically examined their OER performance. $^{351,352}$ They found that the four-fold coordinated $\mathrm{Ni}$ showed better activity compared to single-coordinated $\mathrm{Ni}$ on the zigzag edge, and that both the metal center and its coordination environment play a role in lowering the absorption energy of the reaction intermediates. All the above studies show that the role of the coordinating atoms in SACs is an important OER performance parameter.

More recently, a key study of Ni-based SACs was reported by Feng et al., where one $\mathrm{N}$ atom was replaced with an $\mathrm{S}$ atom in the Ni- $\mathrm{N}_{4}$ moiety and the OER activity outperformed most of the current TM- and/or heteroatom-doped carbon-based electrocatalysts (Fig. 18a and b). ${ }^{353}$ It is well known that $\mathrm{N}$ coordinates more strongly to metal centers than $\mathrm{S}$. The N/S replacement increased the distortion of the SAC, which rendered the $\mathrm{M}-\mathrm{N}_{3} \mathrm{~S}$ moiety chemically more active. In addition, the optimization of the coordinating atoms can also regulate the density-of-states distribution and the adsorption of $\mathrm{OH}$ groups and enhance the electron transfer ability of the metal centers (Fig. 18c). Accordingly, Wu et al. further constructed an $\mathrm{M}-\mathrm{O}_{4}$ moiety, which exhibited an extremely low overpotential of $224 \mathrm{mV}$ at a current density of $10 \mathrm{~mA} \mathrm{~cm}{ }^{-2}$ for the OER (Fig. 18d). ${ }^{354}$ Theoretical studies showed that the stronger electronegativity of the $\mathrm{O}$ atoms is favorable for $\mathrm{OH}^{-}$adsorption, thus leading to the formation of an $\mathrm{Ni}-\mathrm{O}_{4}(\mathrm{OH})_{2}$ configuration with a high oxidation state for the Ni atoms (Fig. 18e and f). Similar studies on coordination-performance relationships have been also reported for SACs with other applications. ${ }^{355}$

The regulation of the coordination environment is an increasingly important research trend for improving the OER activity of SACs. ${ }^{356}$ However, the widely reported SACs with $\mathrm{M}-\mathrm{N}_{4}$ moieties still show limited activity towards the four-electron transfer process of the OER because the strong coordination ability of $\mathrm{N}$ obstructs the electron transfer and adsorption of oxygen groups (e.g., $\mathrm{H}_{2} \mathrm{O}$ and ${ }^{*} \mathrm{OH}$ ). Therefore, partial replacement of $\mathrm{N}$ atoms with other non-metal atoms, such as S, O and P, may provide an alternative option for improving the OER activity. However, the fact that the weak chemical bonding between the metal atoms and $\mathrm{O} / \mathrm{S}$ atoms may reduce the stability should also be considered for these optimization strategies. Finally, we want to highlight that a well-defined coordination environment is of
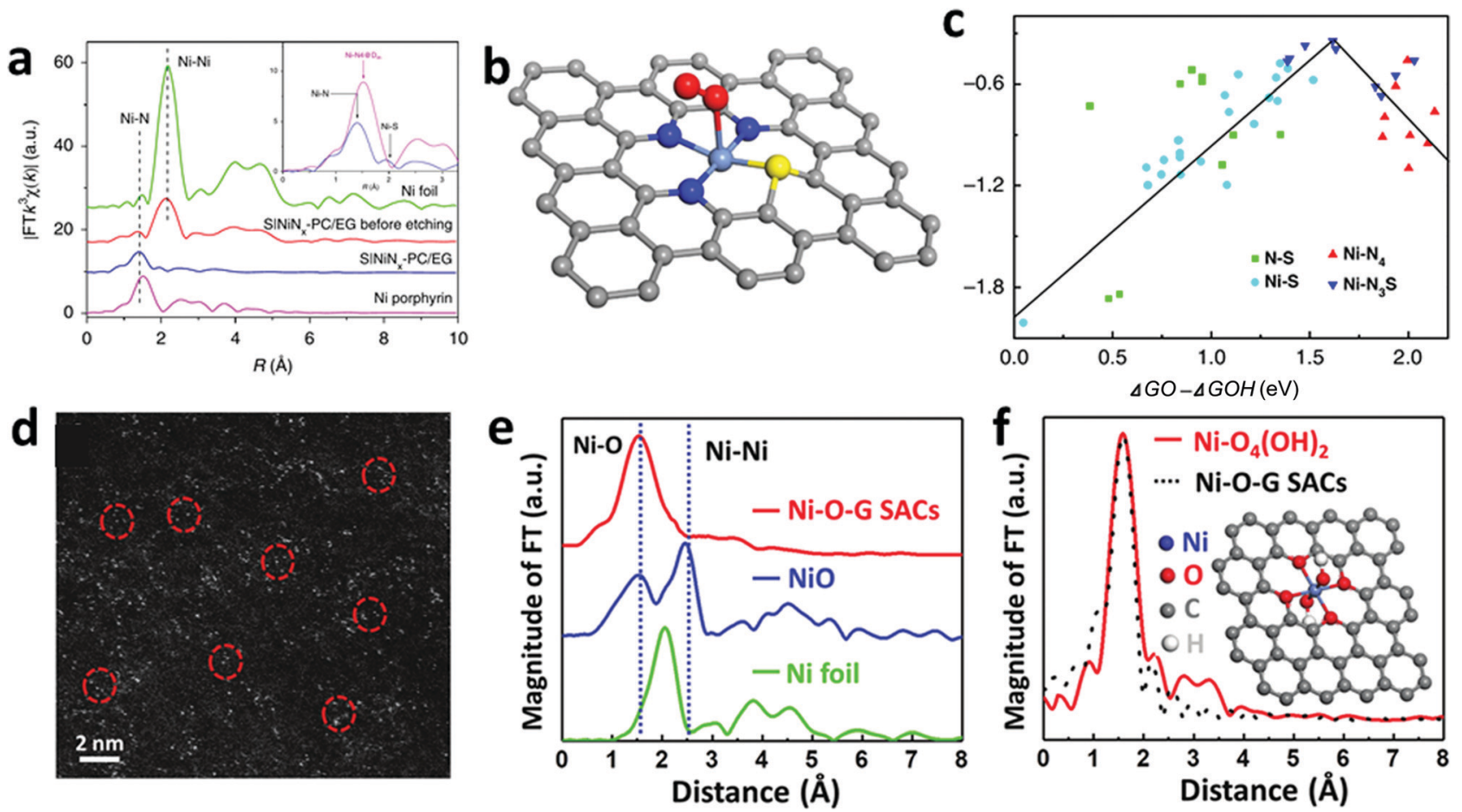

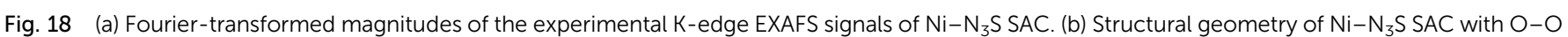

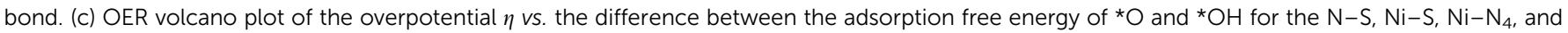

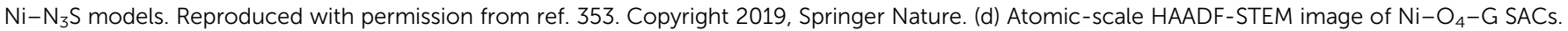

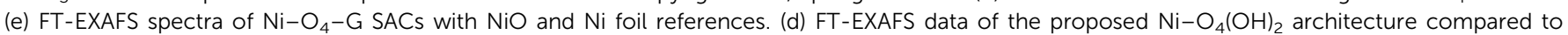
experimental $\mathrm{Ni}-\mathrm{O}_{4}$. Reproduced with permission from ref. 354. Copyright 2020, Wiley VCH. 
great importance for the interpretation of coordination-activity relationships. Thus, we recently introduced a soft-landing molecular strategy to generate well-defined model SAC targets. ${ }^{357}$ The function of $\mathrm{M}-\mathrm{N}_{4}$ moieties could therein be unambiguously investigated in a near-perfect coordination environment.

3.2.3. Single sites vs. dual/multi-site SAC-related systems. As discussed in Sections 1.2.2 and 1.2.3, binuclear and polynuclear molecules are more favorable for water oxidation owning to their synergistic effects and cooperative reaction mechanisms. This also inspires many studies to design dual/multi-site SACs in order to improve their currently unsatisfactory OER performance. As mentioned above, the OER performance of SACs is still far behind molecular OER catalysts despite their high extent of metal center involvement in the catalytic process. This raises two crucial questions for their future: are SACs really suitable for water oxidation? If so, how can we improve their activities? Looking back on traditional TM-based WOCs, the bimetallic systems are generally more active than monometallic ones, as demonstrated for both molecular and heterogeneous catalysis. ${ }^{175,358,359}$ The reasons for this trend may be associated with the fact that the single-site pathway requires higher potentials compared to the dual-site pathway involved in the $\mathrm{I} 2 \mathrm{M}$ mechanism. ${ }^{89}$ Therefore, the exploration of dual or even multi-site SACs is another efficient way to improve the current activities of SACs.
Recently, Wang et al. found that the introduction of $\mathrm{Fe}^{3+}$ into graphene-conjugated molecular $\mathrm{Ni}^{2+}$ (Fig. 19a) could significantly increase the OER activity and stability of the active Ni-Fe sites of the catalyst with a distance of $2.7 \AA$ between the two metal centers. It was observed that both the $\mathrm{Ni}$ and $\mathrm{Fe}$ sites could couple with the $\mathrm{OH}^{-}$ions through terminal and bridging geometries (Fig. 19b and c). Ni-Fe dual sites for water oxidation have been widely studied in traditional heterogeneous WOCs, as discussed in Section 2.2.1; however, this is the first experimental evidence reported to explain the synergistic effects of $\mathrm{Ni}$ and $\mathrm{Fe}$ at the atomic level. ${ }^{360}$ Another important example of a dual site system is the Co-Fe SACs reported by the Hu group. Their study showed that the high activity of Co-Fe SACs arises from a series of structural and coordination evolution processes during the OER in electrolyte containing a certain amount of $\mathrm{Fe}^{3+}$ ions (Fig. 19d). The Co atoms coordinated to the N-doped carbon support first interacted with $\mathrm{OH}^{-} / \mathrm{OH}_{2}$ species in the electrolyte, and then slowly bonded with trace amounts of $\mathrm{Fe}^{3+}$ ions in solution to form the Co-Fe site (Fig. 19e). The enhanced activity was ascribed to the joint functionalities of the Co-Fe-N-C motif. ${ }^{361}$ Although the authors did not elaborate on the detailed reaction mechanism, the in situ XAS data for the formation process of the Co-Fe-N-C site is of great significance to understand the reaction mechanism. Surprisingly, we could not find further studies on dual or multi-site SACs for the OER beyond the two works mentioned
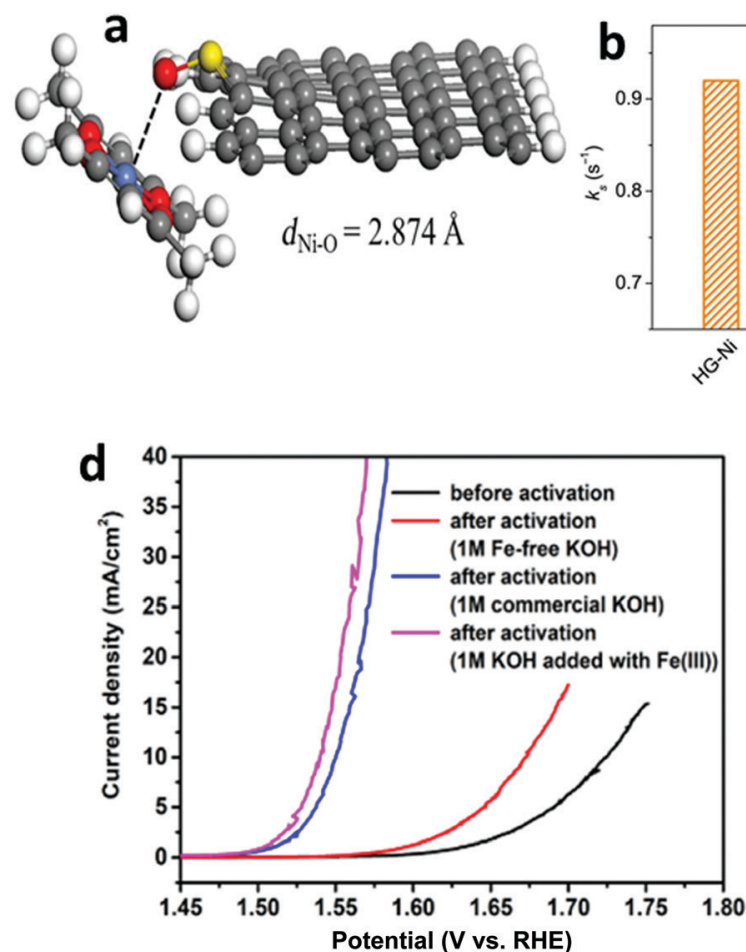
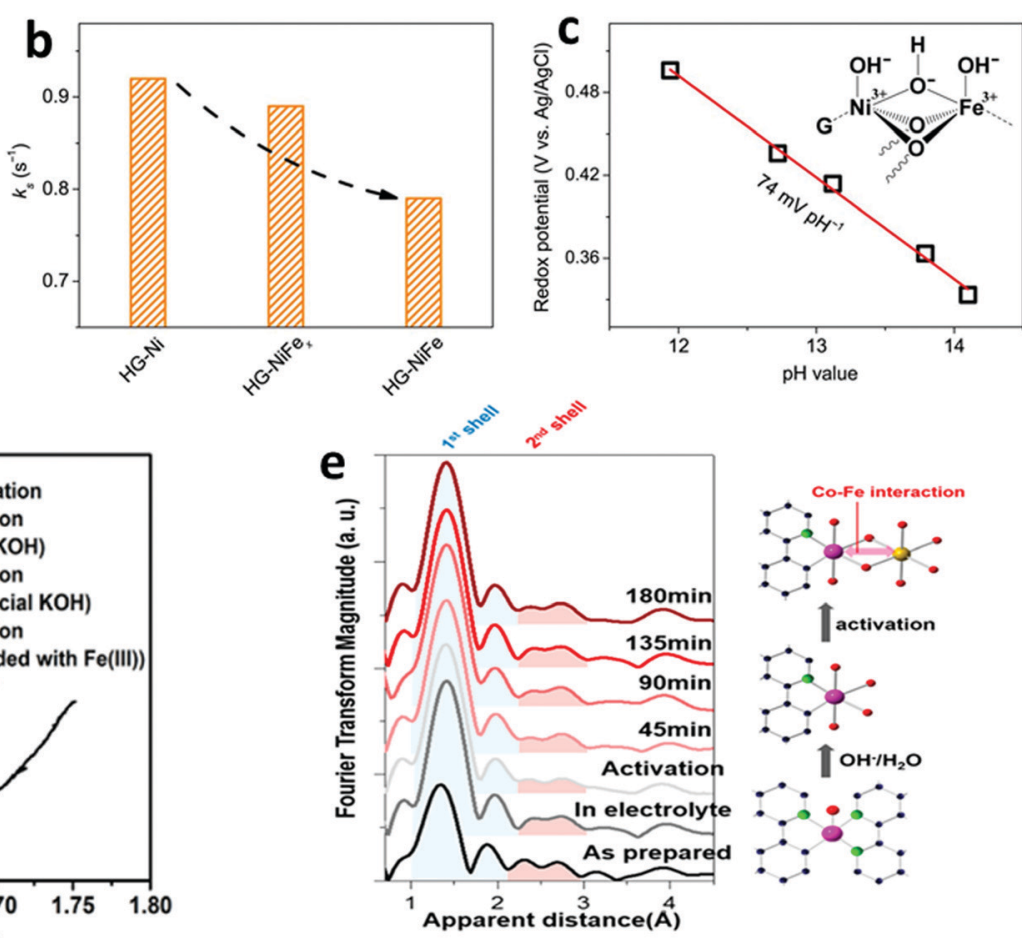

Fig. 19 (a) DFT calculation-derived model of HG-Ni SAC. (b) $k_{\mathrm{s}}$ of metal redox in HG-Ni, HG-NiFe , and HG-NiFe. (c) Redox potentials of Ni-Fe sites versus $\mathrm{pH}$ value of $\mathrm{KOH}$ solution. The inset shows the proposed configuration for the adsorption of $\mathrm{HO}^{-}$ions onto Ni-Fe sites for OER. Reproduced with permission from ref. 360. Copyright 2018 AAAS. (d) LSVs of the Co-N-C (single atom Co dispersed on a N-doped carbon support) with different qualities of $\mathrm{KOH}$ (and different concentrations of $\mathrm{Fe}^{3+}$ ). (e) Fourier transform of $\mathrm{Co} \mathrm{K-edge} \mathrm{EXAFS} \mathrm{spectra} \mathrm{for} \mathrm{as-prepared} \mathrm{Co-} \mathrm{N}-\mathrm{C}$ and the structure changes in $\mathrm{KOH}$ solution with different concentrations of $\mathrm{Fe}^{3+}$ under OER conditions. Reproduced with permission from ref. 361. Copyright 2019, The American Chemical Society. 
above at the time of writing this review. This leaves plenty of room for new explorations because multi-site SACs do not only hold the potential for high performance, but also provide a unique platform for investigating the role of each different metal center in the synergistic interaction of two metal atoms at the atomic level. This holds the key to the much sought-after general structure-performance relationships of WOCs. However, this research is still in its infancy, and the design and synthesis of these SACs are expected to greatly benefit from bi-/poly-nuclear molecular catalyst design concepts, as discussed in Part I.

\subsection{Combining the advantages of molecular and heterogeneous WOCs}

While the previous sections were focused on mutual knowledge transfer between molecular and heterogeneous catalysts, we finally cover emerging hybrid strategies based on the direct coupling of both molecular and heterogeneous WOCs. ${ }^{362,363}$ In addition to the benefits of stabilizing molecular WOCs, as discussed in Section 1.3.2, hybrid systems also provide the opportunity to preserve their inherent advantages, such as synthetic flexibility, atomic-scale monitoring/modeling and structure-function correlations, as elaborated in Part I. They can be combined with the robustness, synthetically convenient tailoring, and favorable application-oriented features of heterogeneous WOCs ( $c f$. Part II). ${ }^{345,364,365}$ More importantly, hybrid systems offer the possibility to overcome the limitations of both catalyst types. ${ }^{366,367}$ In the following, we highlight some key strategies such as hybrid molecular-heterogeneous (photo)electrodes or encapsulation strategies and briefly analyze their major advantages and remaining challenges.

3.3.1. Hybrid molecular-heterogeneous (photo)electrodes. Over the last two decades, many research groups have tried to design hybrid (photo)electrodes by integrating molecular WOCs with conductive or semi-conductive heterogeneous surfaces for electrocatalytic and photoelectrocatalytic processes. ${ }^{368-370}$ In this way, molecular species are largely confined within the diffusion layer near the electrode surface, thus maximizing their participation in the catalytic reaction. ${ }^{371}$ In parallel, the high OER overpotentials of the heterogeneous (photo)electrode were minimized and a higher catalytic output was obtained at constant potentials. $^{372}$ This indicates that hybrid (photo)electrodes can indeed combine advantages from both molecular cocatalysts and heterogeneous substrates. ${ }^{373}$ We have provided an extensive summary of the stabilization of molecular WOCs on heterogeneous substrate surfaces in Section 1.3.2 and we kindly refer to this part for strategies to design these hybrid molecularheterogeneous (photo)electrodes. However, we want to emphasize that in addition to individual engineering of molecular and heterogeneous components, special attention must be paid to engineering the solid-liquid interface of high performance hybrid (photo)electrodes. Accordingly, a wide range of different anchoring strategies have been explored, from expanding physical attachment to chemically tailored anchoring groups. ${ }^{374,375}$ Brudvig and coworkers systematically reviewed different anchoring groups and pointed out some critical factors for selecting those suitable for immobilization. ${ }^{376}$ We want to emphasize here that the stability and the charge transfer capability are two equally important considerations for the anchoring groups. ${ }^{377}$ Future development and evaluation with both principles in mind will significantly facilitate the utilization of molecular WOCs on heterogeneous surfaces. ${ }^{15}$

To facilitate the design and engineering of efficient hybrid devices, one important question has to be answered: what are the individual functions of the heterogeneous substrate and of the immobilized molecular species? For electrocatalytic devices, it is obvious that molecular WOCs are the dominant catalytic centers, especially when an inert substrate is employed. Concerning photoelectrochemical devices, the strong interplay of immobilized molecular cocatalysts and semiconductor substrate demands a stronger separation of their individual functions. ${ }^{378}$ The OER process of photoelectrochemical devices is deeply entangled with charge carrier separation and transportation processes. Therefore, the role of surface-loaded molecular WOCs is expected to be evolutionary rather than stationary. To clarify these complex relationships, our group employed a model system consisting of molecular $\mathrm{Co}_{4} \mathrm{O}_{4}$ cubanes immobilized on a hematite photoanode. ${ }^{379}$ For the first time, we identified the dynamic functionality of the molecular species as a function of the applied potential (Fig. 20a). Briefly, surface cubanes were activated first around the onset potential, and at moderate potentials they started to accumulate large amounts of oxidative equivalents. However, they were unable to transfer them efficiently to the electrolyte at moderate potentials since the competing hematite surface states were more catalytically active. At elevated potentials, the surface molecular WOCs finally started to contribute significantly to the OER. This study highlighted that the role of molecular WOCs in hybrid systems is not necessarily limited to cocatalysts. Instead, their functionality depends on the operational conditions and is closely linked to the properties of the

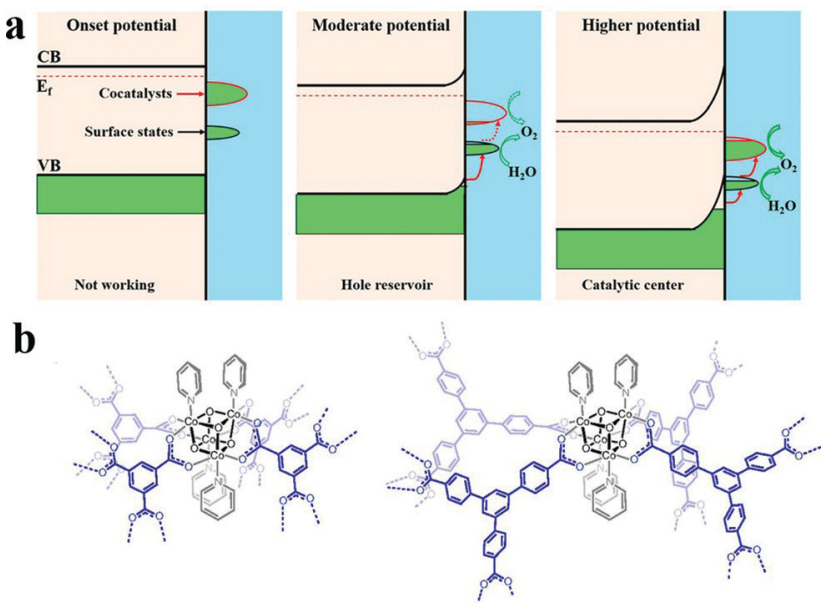

Fig. 20 (a) Dynamic role of molecular cocatalysts on hematite photoanodes upon the modulation of applied potentials. Reproduced with permission from ref. 379. Copyright 2019, The American Chemical Society. (b) Building unit of MOFs with programmable linkers that also function as a protecting ligand for molecular WOCs. Reproduced with permission from ref. 275. Copyright 2019, the National Academy of Sciences. 
heterogeneous substrate. We believe that understanding the interactive relationship between the immobilized molecular species and the underlying heterogeneous materials is crucial for the selection of optimal operation conditions and deserves to be at the center of future studies.

Meanwhile, we have to point out that the development of hybrid systems also faces several major analytical challenges. Just to mention one, it is very demanding to probe their molecular structure after their immobilization. ${ }^{380}$ Similarly, their molecular integrity under operational conditions is another critical issue since both properties are essential for drawing any meaningful mechanistic conclusions, as detailed in Section 1.3. In this context, advanced surface-sensitive and atomic-level resolution techniques, such as HAADF-STEM, AC-TEM and XAS, are important to clarify these questions, as outlined in Section 1.1.1.

3.3.2. Encapsulation of molecular species into heterogeneous scaffolds. As discussed in Section 1.3.2, encapsulation is another emerging strategy for integrating molecular and heterogeneous catalysts. ${ }^{381,382}$ In addition, encapsulation also combines the structural flexibility of both the molecular 'guest' and heterogeneous 'host'. Therefore, this strategy benefits fully from progress in synthetic chemistry and holds great promise. Typically, porous materials such as zeolites, MOFs and covalent organic frameworks (COFs) are used as the heterogeneous scaffold. ${ }^{383}$ Molecular species are either directly incorporated into the nano-voids of these porous materials or used as building blocks for the formation of novel frameworks. ${ }^{384-386}$ These strategies are based on the assumption that the nanoscale confinement effect disables the intermolecular deactivation routes of molecular WOCs. Therefore, many of these systems offer the benefit of investigating molecular reaction pathways, while circumventing the issue of aggregationinduced destabilization. In a representative study, Tilley and coworkers directly assembled $\mathrm{Co}_{4} \mathrm{O}_{4}$ cubanes as part of MOFs, thereby preventing their intermolecular decomposition (Fig. 20b). ${ }^{275}$ This approach enabled the isolation and observation of reaction intermediates of the molecular species, and a cofacial oxo-hydroxo coupling pathway was proposed. Additionally, the reactivity and stability of molecular cubanes could be regulated by using different organic linkers for the framework, which transfers many design strategies from porous materials to the field of WOC research. In another study by Kern et al., heterobimetallic catalysts were prepared through a supramolecular assembly approach, where porphyrin macrocycles were employed as the host sites for different metal centers $\left(\mathrm{Co}^{2+}\right.$ and $\left.\mathrm{Fe}^{2+}\right){ }^{387}$ These heterobimetallic catalysts showed a nonlinear increase in OER activity (by two orders of magnitude) compared to the corresponding homometallic systems. More importantly, the employed combinational strategy offers broad tailoring opportunities such as selection of different metal species, exploration of bi- and multimetallic interactions, and substitution of porphyrin macrocycles with different ligands. However, these encapsulated heterobimetallic sites still exhibit limited electrocatalytic activity, which is due to the poor conductivity of the organic motifs in the porous materials. ${ }^{388}$ Redox-active linkers can be considered as an alternative here to enhance the charge transportation properties. Finally, it should be considered that encapsulation does not necessarily warrant the operational stability of molecular species. Thus, encapsulated molecular WOCs should still be subjected to careful pre- and post-catalytic characterizations.

Overall, molecular-heterogeneous hybrid systems keep attracting interdisciplinary attention. Their advancement is not only of interest to promote industrial-scale AP with new components and building blocks, but also to explore fundamental aspects of catalyst design and stability. More importantly, these "crossover" studies are pushing the conventional boundaries between molecular and heterogeneous catalysts, which will considerably expedite the knowledge transfer and progress in catalyst optimization.

\subsection{Enhanced knowledge transfer with advanced characterization techniques}

Since the transfer of knowledge between molecular and heterogeneous WOCs relies on structural similarities at the atomic level, detailed atomic resolution models of both catalyst types are indispensable. Generally, this poses no special challenges for molecular WOCs due to the widely applicable crystallization and X-ray crystallography methods. However, for heterogeneous WOCs, this task is more problematic since most catalytically active solids are not perfectly crystalline materials, but contain many structural defects up to bulk disorder or amorphization. In this case, advanced characteristic techniques such as XAS, XFEL diffraction and PDF analysis are crucial for investigating the coordination environment and electronic structure of solid catalysts. Detailed structural insight into the surfaces or edges of heterogeneous WOCs is decisive for knowledge transfer to the molecular field, especially for the selective tailoring of molecular WOCs mimicking the motifs of efficient heterogeneous WOCs. Consequently, the as-constructed molecular WOCs can serve as important models for an in-depth understanding of the detailed reaction mechanisms of the original heterogeneous WOCs. Here, we can benefit from the wide accessibility of lab-scale spectroscopy for molecular WOCs, such as UV-Vis, Raman, EPS, and FTIR spectroscopy. Furthermore, the abovementioned methodological repertoire is also vital to verify the successful transfer of specially designed molecular features to the surfaces of a solid catalyst. For more experimental and theoretical details of these advanced characterization techniques, we kindly refer readers to our previous review. ${ }^{23}$

As we pointed out above, operando measurements are at the center of identifying active intermediates and of ultimately uncovering the reaction mechanism. To date, we have a wide arsenal for operando tests, ranging from in-house spectroscopy (ATR-FTIR, Raman, EPR, etc.) and advanced microscopy (HRTEM, STEM, and STM) to synchrotron-based high-energy spectroscopic techniques. In addition to the identification of intermediates, most of these operando measurements can provide time-resolved information about the active intermediates on the way to understanding the full reaction kinetics. More importantly, some operando techniques, e.g. XAS and HRTEM, 
directly monitor detailed structural changes, and thus enabled exciting breakthroughs in recent years. ${ }^{274,276}$ The observed dynamic catalyst transformations illustrate the need for new, evolutionary concepts to point out parallels between molecular and heterogeneous WOCs. Here, it seems that growing insight into the mechanisms on both sides strengthens the bridges that connect the molecular and heterogeneous realms, especially in water oxidation catalysis, which finally, is a process revolving around the transformation of small molecules at specific catalytic centers. However, we have to make it clear that even with so many advanced operando techniques at our disposal, we need more studies to strengthen the inspirational continuous knowledge exchange between both catalytic research areas. In parallel, another knowledge gap is opening up at the interfaces of molecular and heterogeneous assemblies. These challenges will remain until we can unambiguously monitor, predict and explain the dynamic nature of catalytic processes.

\section{Conclusions}

Since the increasing worldwide need for clean energy has turned AP into a research hotspot, tremendous efforts have been devoted to the optimization of WOCs as a major bottleneck. Over the past decades, molecular and heterogeneous strategies in parallel have brought forward enormous progress in the design of new respective WOCs. Since they greatly facilitate mechanistic and structural tracking at the atomic level, molecular WOCs serve as model systems to investigate the versatile interplay and influence of both metal centers and ligands on the reaction pathways and catalytic activity. This line of research is now being intensified for molecular WOCs with higher nuclearities to benefit from their specific advantages and similarities to heterogeneous surface motifs. Meanwhile, rapid progress has been made in the mechanistic study of heterogeneous WOCs despite the analytical challenges still associated with the operando monitoring of their active sites. Now that increasing insight into the separate advantages and drawbacks of molecular and heterogeneous WOCs arising from their respective dimensionalities is available, at least four strategic approaches for knowledge transfer and their direct combination into hybrid systems are emerging. (1) The discovery of joint structural motifs of both molecular WOCs and active heterogeneous surfaces greatly promotes unification of design principles and mechanistic models from both sides in the search for next-generation WOCs. For instance, the translation of the most active surface structural features of heterogeneous WOCs into molecular analogues will facilitate their mechanistic investigation on the atomic scale with convenient analytical methods. (2) In turn, applying structure-activity relationships of molecular WOCs to the construction of heterogeneous systems can expedite the direct clarification of open questions. This is particularly applicable for optimizing the interactions of active metal sites and supports/ligands, as in the emerging field of SACs. Their in-depth study holds the key to fully understand the coordinative needs and reaction pathways of single (and dual) metal sites in diverse heterogeneous environments. (3) Furthermore, this crossover research can help to draw or erase the lines between heterogenized molecular systems, such as SACs, and low-degree doping of solids. (4) Moreover, the targeted construction of hybrid and encapsulated catalyst systems holds the key to combine the analytical accessibility and synthetic flexibility of molecular WOCs with the economic robustness of heterogeneous supports.

The investigation of the dynamic roles of both components in these hybrids, e.g. the transitions of molecular cocatalysts on photoelectrodes or the participation of support atoms in single and dual-site SAC reactions, has just begun and will deliver further crucial insights. We want to highlight that this line of research requires rather demanding cutting-edge operando measurements. These investigations are indispensable for crucial snapshots of the catalytic process not only for understanding the reaction mechanism, but also to accelerate the dynamic mutual evolution of both molecular and heterogeneous WOCs. Ultimately, understanding these complex processes will help us combine the best of both realms and to circumvent the individual drawbacks of both catalyst types. Since the quest for applied AP is ongoing, crossing the borders between the different catalytic divisions is a key step to expedite the search for durable and technologically relevant catalysts.

\section{Conflicts of interest}

There are no conflicts to declare.

\section{Acknowledgements}

This work was supported by the University Research Priority Program (URPP) for Solar Light to Chemical Energy Conversion (LightChEC) and by the Swiss National Science Foundation (Sinergia Grant No. CRSII2_160801/1). W. Wan thanks the China Scholarship Council (CSC) for his PhD fellowship. S. Heidari thanks the University of Zurich for support of her postdoctoral research with a Forschungskredit grant (Grant No. FK-18-106).

\section{References}

1 M. D. Kärkäs and B. Åkermark, Dalton Trans., 2016, 45, 14421.

2 S. Berardi, S. Drouet, L. Francàs, C. Gimbert-Suriñach, M. Guttentag, C. Richmond, T. Stoll and A. Llobet, Chem. Soc. Rev., 2014, 43, 7501.

3 B. M. Hunter, H. B. Gray and A. M. Müller, Chem. Rev., 2016, 116, 14120.

4 T. J. Meyer, M. V. Sheridan and B. D. Sherman, Chem. Soc. Rev., 2017, 46, 6148.

5 C. C. L. McCrory, S. Jung, J. C. Peters and T. F. Jaramillo, J. Am. Chem. Soc., 2013, 135, 16977.

6 A. Singh and L. Spiccia, Coord. Chem. Rev., 2013, 257, 2607. 
7 A. R. Parent, R. H. Crabtree and G. W. Brudvig, Chem. Soc. Rev., 2013, 42, 2247.

8 R. Matheu, P. Garrido-Barros, M. Gil-Sepulcre, M. Z. Ertem, X. Sala, C. Gimbert-Suriñach and A. Llobet, Nat. Rev. Chem., 2019, 3, 331.

9 A. Bergmann, E. Martinez-Moreno, D. Teschner, P. Chernev, M. Gliech, J. F. de Araújo, T. Reier, H. Dau and P. Strasser, Nat. Commun., 2015, 6, 8625.

10 D. B. Eremin and V. P. Ananikov, Coord. Chem. Rev., 2017, 346, 2.

11 H. Dau, C. Limberg, T. Reier, M. Risch, S. Roggan and P. Strasser, ChemCatChem, 2010, 2, 724.

12 M. Risch, F. Ringleb, M. Kohlhoff, P. Bogdanoff, P. Chernev, I. Zaharieva and H. Dau, Energy Environ. Sci., 2015, 8, 661.

13 B. Li, F. Li, S. Bai, Z. Wang, L. Sun, Q. Yang and C. Li, Energy Environ. Sci., 2012, 5, 8229.

14 C. Pasquini, I. Zaharieva, D. González-Flores, P. Chernev, M. R. Mohammadi, L. Guidoni, R. D. L. Smith and H. Dau, J. Am. Chem. Soc., 2019, 141, 2938.

15 B. Zhang and L. Sun, Chem. Soc. Rev., 2019, 48, 2216.

16 D. Zhao, Z. Zhuang, X. Cao, C. Zhang, Q. Peng, C. Chen and Y. Li, Chem. Soc. Rev., 2020, 49, 2215.

17 M. A. Newton, Catal., Struct. React., 2017, 3, 2.

18 E. de La Mora, N. Coquelle, C. S. Bury, M. Rosenthal, J. M. Holton, I. Carmichael, E. F. Garman, M. Burghammer, J.-P. Colletier and M. Weik, Proc. Natl. Acad. Sci. U. S. A., 2020, 117, 4142.

19 A. Tanaka, Y. Fukushima and N. Kamiya, J. Am. Chem. Soc., 2017, 139, 1718.

20 J. Huang, J. D. Blakemore, D. Fazi, O. Kokhan, N. D. Schley, R. H. Crabtree, G. W. Brudvig and D. M. Tiede, Phys. Chem. Chem. Phys., 2014, 16, 1814.

21 K. R. Yang, A. J. Matula, G. Kwon, J. Hong, S. W. Sheehan, J. M. Thomsen, G. W. Brudvig, R. H. Crabtree, D. M. Tiede, L. X. Chen and V. S. Batista, J. Am. Chem. Soc., 2016, 138, 5511.

22 C. A. Triana, R. Moré, A. J. Bloomfield, P. V. Petrović, S. G. Ferrón, G. Stanley, S. D. Zarić, T. Fox, E. N. Brothers, S. W. Sheehan, P. T. Anastas and G. R. Patzke, Matter, 2019, 1, 1354.

23 J. Li, R. Güttinger, R. Moré, F. Song, W. Wan and G. R. Patzke, Chem. Soc. Rev., 2017, 46, 6124.

24 C. Glöckner, J. Kern, M. Broser, A. Zouni, V. Yachandra and J. Yano, J. Biol. Chem., 2013, 288, 22607.

25 S. A. Bartlett, E. V. Sackville, E. K. Gibson, V. Celorrio, P. P. Wells, M. Nachtegaal, S. W. Sheehan and U. Hintermair, Chem. Commun., 2019, 55, 7832.

26 F. Song, R. Moré, M. Schilling, G. Smolentsev, N. Azzaroli, T. Fox, S. Luber and G. R. Patzke, J. Am. Chem. Soc., 2017, 139, 14198.

27 R. Schiwon, K. Klingan, H. Dau and C. Limberg, Chem. Commun., 2014, 50, 100.

28 D. Lebedev, Y. Pineda-Galvan, Y. Tokimaru, A. Fedorov, N. Kaeffer, C. Copéret and Y. Pushkar, J. Am. Chem. Soc., 2018, 140, 451.

29 M. Haruta, K. Yoshida, H. Kurata and S. Isoda, Ultramicroscopy, 2008, 108, 545.
30 M. Haruta, K. Yoshida, H. Kurata and S. Isoda, Mol. Cryst. Liq. Cryst., 2008, 492, 200.

31 Y. Zhao, K. R. Yang, Z. Wang, X. Yan, S. Cao, Y. Ye, Q. Dong, X. Zhang, J. E. Thorne, L. Jin, K. L. Materna, A. Trimpalis, H. Bai, S. C. Fakra, X. Zhong, P. Wang, X. Pan, J. Guo, M. Flytzani-Stephanopoulos, G. W. Brudvig, V. S. Batista and D. Wang, Proc. Natl. Acad. Sci. U. S. A., 2018, 115, 2902.

32 Y. Yu, Catal. Today, 2020, 350, 192.

33 B. Zhang and L. Sun, J. Am. Chem. Soc., 2019, 141, 5565.

34 M. D. Kärkäs, R.-Z. Liao, T. M. Laine, T. Åkermark, S. Ghanem, P. E. M. Siegbahn and B. Åkermark, Catal. Sci. Technol., 2016, 6, 1306.

35 Y. Yan, J. S. Lee and D. A. Ruddy, Inorg. Chem., 2015, 54, 4550.

36 N.-Y. Huang, J.-Q. Shen, Z.-M. Ye, W.-X. Zhang, P.-Q. Liao and X.-M. Chen, Chem. Sci., 2019, 10, 9859.

37 M. Kondo and S. Masaoka, Chem. Lett., 2016, 45, 1220.

38 P. Garrido-Barros, C. Gimbert-Suriñach, R. Matheu, X. Sala and A. Llobet, Chem. Soc. Rev., 2017, 46, 6088.

39 J. D. Blakemore, R. H. Crabtree and G. W. Brudvig, Chem. Rev., 2015, 115, 12974.

40 J. L. Fillol, Z. Codolà, I. Garcia-Bosch, L. Gómez, J. J. Pla and M. Costas, Nat. Chem., 2011, 3, 807.

41 W. C. Ellis, N. D. McDaniel, S. Bernhard and T. J. Collins, J. Am. Chem. Soc., 2010, 132, 10990.

42 Z. Codolà, L. Gómez, S. T. Kleespies, L. Que, M. Costas and J. Lloret-Fillol, Nat. Commun., 2015, 6, 5865.

43 B. Das, A. Orthaber, S. Ott and A. Thapper, ChemSusChem, 2016, 9, 1178.

44 G. Panchbhai, W. M. Singh, B. Das, R. T. Jane and A. Thapper, Eur. J. Inorg. Chem., 2016, 3262.

45 B. Yang, Q.-Q. Yang, X. Jiang, B. Chen, C.-H. Tung and L.-Z. Wu, Chem. Commun., 2017, 53, 9063.

46 Z. Codolà, I. Gamba, F. Acuña-Parés, C. Casadevall, M. Clémancey, J.-M. Latour, J. M. Luis, J. Lloret-Fillol and M. Costas, J. Am. Chem. Soc., 2019, 141, 323.

47 S. I. Shylin, M. V. Pavliuk, L. D’Amario, F. Mamedov, J. Sá, G. Berggren and I. O. Fritsky, Chem. Commun., 2019, 55, 3335.

48 D. Das, S. Pattanayak, K. K. Singh, B. Garai and S. Sen Gupta, Chem. Commun., 2016, 52, 11787.

49 S. Hong, F. F. Pfaff, E. Kwon, Y. Wang, M.-S. Seo, E. Bill, K. Ray and W. Nam, Angew. Chem., Int. Ed., 2014, 53, 10403.

50 B. Wang, Y.-M. Lee, W.-Y. Tcho, S. Tussupbayev, S.-T. Kim, Y. Kim, M. S. Seo, K.-B. Cho, Y. Dede, B. C. Keegan, T. Ogura, S. H. Kim, T. Ohta, M.-H. Baik, K. Ray, J. Shearer and W. Nam, Nat. Commun., 2017, 8, 14839.

51 W. Sinha, A. Mizrahi, A. Mahammed, B. Tumanskii and Z. Gross, Inorg. Chem., 2018, 57, 478.

52 L. Xu, H. Lei, Z. Zhang, Z. Yao, J. Li, Z. Yu and R. Cao, Phys. Chem. Chem. Phys., 2017, 19, 9755.

53 J.-W. Wang, W. Liu, D. Zhong and T. Lu, Coord. Chem. Rev., 2019, 378, 237.

54 J. Lin, Q. Han and Y. Ding, Chem. Rec., 2018, 18, 1531.

55 Y.-Y. Li, L.-P. Tong and R.-Z. Liao, Inorg. Chem., 2018, 57,4590 . 
56 A. Q. Mir, G. Joshi, P. Ghosh, S. Khandelwal, A. Kar, R. Hegde, S. Khatua and A. Dutta, ACS Energy Lett., 2019, 4, 2428.

57 C. Lu, J. Du, X.-J. Su, M.-T. Zhang, X. Xu, T. J. Meyer and Z. Chen, ACS Catal., 2016, 6, 77.

58 R.-J. Xiang, H.-Y. Wang, Z.-J. Xin, C.-B. Li, Y.-X. Lu, X.-W. Gao, H.-M. Sun and R. Cao, Chem. - Eur. J., 2016, 22, 1602.

59 G.-Y. Luo, H.-H. Huang, J.-W. Wang and T.-B. Lu, ChemSusChem, 2016, 9, 485.

60 S. J. Koepke, K. M. Light, P. E. VanNatta, K. M. Wiley and M. T. Kieber-Emmons, J. Am. Chem. Soc., 2017, 139, 8586.

61 M. Schilling, G. R. Patzke, J. Hutter and S. Luber, J. Phys. Chem. C, 2016, 120, 7966.

62 H. Pan, L. Duan and R.-Z. Liao, ChemCatChem, 2020, 12, 219.

63 Ł. Szyrwiel, D. Lukács, D. F. Srankó, Z. Kerner, A. Kotynia, J. Brasuń, B. Setner, Z. Szewczuk, K. Malec and J. S. Pap, RSC Adv., 2017, 7, 24657.

64 J. Wang, H. Huang and T. Lu, Chin. J. Chem., 2017, 35, 586.

65 F. Chen, N. Wang, H. Lei, D. Guo, H. Liu, Z. Zhang, W. Zhang, W. Lai and R. Cao, Inorg. Chem., 2017, 56, 13368.

66 Y. Liu, Y. Han, Z. Zhang, W. Zhang, W. Lai, Y. Wang and R. Cao, Chem. Sci., 2019, 10, 2613.

67 B. Ariela, W. Yaniv, S. Dror, K. Haya, A. Yael, M. Eric and M. Dan, Dalton Trans., 2017, 46, 10774.

68 D. Wang and C. O. Bruner, Inorg. Chem., 2017, 56, 13638.

69 H. Kuilya, N. Alam, D. Sarma, D. Choudhury and A. Kalita, Chem. Commun., 2019, 55, 5483.

70 T. Ghosh, P. Ghosh and G. Maayan, ACS Catal., 2018, 8, 10631.

71 Q.-Y. Mao, Y.-J. Pang, X.-C. Li, G.-J. Chen and H.-W. Tan, ACS Catal., 2019, 9, 8798.

72 M.-C. Kafentzi, R. Papadakis, F. Gennarini, A. Kochem, O. Iranzo, Y. Le Mest, N. Le Poul, T. Tron, B. Faure, A. J. Simaan and M. Réglier, Chem. - Eur. J., 2018, 24, 5213.

73 M. Yoshida, M. Kondo, M. Okamura, M. Kanaike, S. Haesuwannakij, H. Sakurai and S. Masaoka, Faraday Discuss., 2017, 198, 181.

74 P. Xu, S. Hu, H.-D. Zhang and X. Zheng, Phys. Chem. Chem. Phys., 2018, 20, 14919.

75 H. Wang, Z. Xin, R. Xiang, S. Liu, X. Gao and C. Li, Chin. J. Chem., 2016, 34, 757.

76 S. Liu, Y.-J. Lei, Z.-J. Xin, R.-J. Xiang, S. Styring, A. Thapper and H.-Y. Wang, Int. J. Hydrogen Energy, 2017, 42, 29716.

77 P. Garrido-Barros, S. Grau, S. Drouet, J. Benet-Buchholz, C. Gimbert-Suriñach and A. Llobet, ACS Catal., 2019, 9, 3936.

78 A. Annunziata, R. Esposito, G. Gatto, M. E. Cucciolito, A. Tuzi, A. Macchioni and F. Ruffo, Eur. J. Inorg. Chem., 2018, 3304.

79 J.-W. Wang, X.-Q. Zhang, H.-H. Huang and T.-B. Lu, ChemCatChem, 2016, 8, 3287.

80 S. W. Gersten, G. J. Samuels and T. J. Meyer, J. Am. Chem. Soc., 1982, 104, 4029.
81 J. Limburg, J. S. Vrettos, L. M. Liable-Sands, A. L. Rheingold, R. H. Crabtree and G. W. Brudvig, Science, 1999, 283, 1524.

82 R. Angamuthu, P. Byers, M. Lutz, A. L. Spek and E. Bouwman, Science, 2010, 327, 313.

83 J.-W. Wang, D.-C. Zhong and T.-B. Lu, Coord. Chem. Rev., 2018, 377, 225.

84 R. H. Holm, P. Kennepohl and E. I. Solomon, Chem. Rev., 1996, 96, 2239.

85 C. E. Elwell, N. L. Gagnon, B. D. Neisen, D. Dhar, A. D. Spaeth, G. M. Yee and W. B. Tolman, Chem. Rev., 2017, 117, 2059.

86 R. Dhiman and C. M. Nagaraja, Eur. J. Inorg. Chem., 2018, 2826.

87 H. M. C. Lant, T. K. Michaelos, L. S. Sharninghausen, B. Q. Mercado, R. H. Crabtree and G. W. Brudvig, Eur. J. Inorg. Chem., 2019, 2115.

88 S. Neudeck, S. Maji, I. López, S. Meyer, F. Meyer and A. Llobet, J. Am. Chem. Soc., 2014, 136, 24.

89 D. W. Shaffer, Y. Xie and J. J. Concepcion, Chem. Soc. Rev., 2017, 46, 6170.

90 C. Sens, I. Romero, M. Rodríguez, A. Llobet, T. Parella and J. Benet-Buchholz, J. Am. Chem. Soc., 2004, 126, 7798.

91 F. Bozoglian, S. Romain, M. Z. Ertem, T. K. Todorova, C. Sens, J. Mola, M. Rodríguez, I. Romero, J. BenetBuchholz, X. Fontrodona, C. J. Cramer, L. Gagliardi and A. Llobet, J. Am. Chem. Soc., 2009, 131, 15176.

92 S. Romain, F. Bozoglian, X. Sala and A. Llobet, J. Am. Chem. Soc., 2009, 131, 2768.

93 M. L. Rigsby, S. Mandal, W. Nam, L. C. Spencer, A. Llobet and S. S. Stahl, Chem. Sci., 2012, 3, 3058.

94 J. Limburg, J. S. Vrettos, H. Chen, J. C. de Paula, R. H. Crabtree and G. W. Brudvig, J. Am. Chem. Soc., 2001, 123, 423.

95 T. Liu, B. Zhang and L. Sun, Chem. - Asian J., 2019, 14, 31. 96 E. A. Karlsson, B.-L. Lee, T. Åkermark, E. V. Johnston, M. D. Kärkäs, J. Sun, Ö. Hansson, J.-E. Bäckvall and B. Åkermark, Angew. Chem., Int. Ed., 2011, 50, 11715.

97 W. A. A. Arafa, M. D. Kärkäs, B.-L. Lee, T. Åkermark, R.Z. Liao, H.-M. Berends, J. Messinger, P. E. M. Siegbahn and B. Åkermark, Phys. Chem. Chem. Phys., 2014, 16, 11950.

98 S. Fukuzumi, S. Mandal, K. Mase, K. Ohkubo, H. Park, J. Benet-Buchholz, W. Nam and A. Llobet, J. Am. Chem. Soc., 2012, 134, 9906.

99 C. Gimbert-Suriñach, D. Moonshiram, L. Francàs, N. Planas, V. Bernales, F. Bozoglian, A. Guda, L. Mognon, I. López, M. A. Hoque, L. Gagliardi, C. J. Cramer and A. Llobet, J. Am. Chem. Soc., 2016, 138, 15291.

100 H. Kotani, D. Hong, K. Satonaka, T. Ishizuka and T. Kojima, Inorg. Chem., 2019, 58, 3676.

101 T. Ishizuka, A. Watanabe, H. Kotani, D. Hong, K. Satonaka, T. Wada, Y. Shiota, K. Yoshizawa, K. Ohara, K. Yamaguchi, S. Kato, S. Fukuzumi and T. Kojima, Inorg. Chem., 2016, $55,1154$.

102 J.-W. Wang, P. Sahoo and T.-B. Lu, ACS Catal., 2016, 6, 5062 . 
103 H.-Y. Wang, E. Mijangos, S. Ott and A. Thapper, Angew. Chem., Int. Ed., 2014, 53, 14499.

104 R. Modak, B. Mondal, Y. Sikdar, J. Banerjee, E. Colacio, I. Oyarzabal, J. Cano and S. Goswami, Dalton Trans., 2020, 49, 6328.

105 D. Wang and L. Que, Chem. Commun., 2013, 49, 10682.

106 C. Panda, J. Debgupta, D. Díaz Díaz, K. K. Singh, S. Sen Gupta and B. B. Dhar, J. Am. Chem. Soc., 2014, 136, 12273.

107 W.-P. To, T. Wai-Shan Chow, C.-W. Tse, X. Guan, J.-S. Huang and C.-M. Che, Chem. Sci., 2015, 6, 5891.

108 Y. Liu, R. Xiang, X. Du, Y. Ding and B. Ma, Chem. Commun., 2014, 50, 12779.

109 B. Das, B.-L. Lee, E. A. Karlsson, T. Åkermark, A. Shatskiy, S. Demeshko, R.-Z. Liao, T. M. Laine, M. Haukka, E. Zeglio, A. F. Abdel-Magied, P. E. M. Siegbahn, F. Meyer, M. D. Kärkäs, E. V. Johnston, E. Nordlander and B. Åkermark, Dalton Trans., 2016, 45, 13289.

110 L. D. Wickramasinghe, R. Zhou, R. Zong, P. Vo, K. J. Gagnon and R. P. Thummel, J. Am. Chem. Soc., 2015, 137, 13260.

111 K. G. Kottrup, S. D’Agostini, P. H. van Langevelde, M. A. Siegler and D. G. H. Hetterscheid, ACS Catal., 2018, 8, 1052.

112 C. M. Lemon, M. Huynh, A. G. Maher, B. L. Anderson, E. D. Bloch, D. C. Powers and D. G. Nocera, Angew. Chem., Int. Ed., 2016, 55, 2176.

113 S. Karim, A. Chakraborty, D. Samanta, E. Zangrando, T. Ghosh and D. Das, Catal. Sci. Technol., 2020, 10, 2830.

114 H. Lee, X. Wu and L. Sun, Nanoscale, 2020, 12, 4187.

115 X.-J. Su, M. Gao, L. Jiao, R.-Z. Liao, P. E. M. Siegbahn, J.-P. Cheng and M.-T. Zhang, Angew. Chem., Int. Ed., 2015, 54, 4909.

116 Q.-Q. Hu, X.-J. Su and M.-T. Zhang, Inorg. Chem., 2018, 57, 10481.

117 X. Zhang, Y.-Y. Li, J. Jiang, R. Zhang, R.-Z. Liao and M. Wang, Inorg. Chem., 2020, 59, 5424.

118 A. Sartorel, M. Bonchio, S. Campagna and F. Scandola, Chem. Soc. Rev., 2013, 42, 2262.

119 H. B. Lee, D. A. Marchiori, R. Chatterjee, P. H. Oyala, J. Yano, R. D. Britt and T. Agapie, J. Am. Chem. Soc., 2020, 142, 3753.

120 N. Cox, M. Retegan, F. Neese, D. A. Pantazis, A. Boussac and W. Lubitz, Science, 2014, 345, 804.

121 M. Askerka, G. W. Brudvig and V. S. Batista, Acc. Chem. Res., 2017, 50, 41.

122 G. C. Dismukes, R. Brimblecombe, G. A. N. Felton, R. S. Pryadun, J. E. Sheats, L. Spiccia and G. F. Swiegers, Acc. Chem. Res., 2009, 42, 1935.

123 R. G. Hadt, D. Hayes, C. N. Brodsky, A. M. Ullman, D. M. Casa, M. H. Upton, D. G. Nocera and L. X. Chen, J. Am. Chem. Soc., 2016, 138, 11017.

124 J. G. McAlpin, Y. Surendranath, M. Dinca, T. A. Stich, S. A. Stoian, W. H. Casey, D. G. Nocera and R. D. Britt, J. Am. Chem. Soc., 2010, 132, 6882.

125 J. G. McAlpin, T. A. Stich, C. A. Ohlin, Y. Surendranath, D. G. Nocera, W. H. Casey and R. D. Britt, J. Am. Chem. Soc., 2011, 133, 15444.
126 C. N. Brodsky, R. G. Hadt, D. Hayes, B. J. Reinhart, N. Li, L. X. Chen and D. G. Nocera, Proc. Natl. Acad. Sci. U. S. A., 2017, 114, 3855.

127 H. Liu and H. Frei, ACS Catal., 2020, 10, 2138.

128 F. Evangelisti, R. Moré, F. Hodel, S. Luber and G. R. Patzke, J. Am. Chem. Soc., 2015, 137, 11076.

129 A. I. Nguyen, D. L. M. Suess, L. E. Darago, P. H. Oyala, D. S. Levine, M. S. Ziegler, R. D. Britt and T. D. Tilley, J. Am. Chem. Soc., 2017, 139, 5579.

130 A. I. Nguyen, L. E. Darago, D. Balcells and T. D. Tilley, J. Am. Chem. Soc., 2018, 140, 9030.

131 X. Jiang, J. Li, B. Yang, X.-Z. Wei, B.-W. Dong, Y. Kao, M.-Y. Huang, C.-H. Tung and L.-Z. Wu, Angew. Chem., 2018, 57, 7850.

132 W.-S. Gao, J.-M. Wang, N.-N. Shi, C.-N. Chen, Y.-H. Fan and M. Wang, New J. Chem., 2019, 43, 4640.

133 J. Lin, X. Liang, X. Cao, N. Wei and Y. Ding, Chem. Commun., 2018, 54, 12515.

134 J.-M. Wang, Y.-R. Liu, X.-Y. Mao, N.-N. Shi, X. Zhang, H.-S. Wang, Y.-H. Fan and M. Wang, Chem. - Asian J., 2019, 14, 2685.

135 M. Okamura, M. Kondo, R. Kuga, Y. Kurashige, T. Yanai, S. Hayami, V. K. K. Praneeth, M. Yoshida, K. Yoneda, S. Kawata and S. Masaoka, Nature, 2016, 530, 465.

136 R.-Z. Liao, S. Masaoka and P. E. M. Siegbahn, ACS Catal., 2018, 8, 11671.

137 G. Maayan, N. Gluz and G. Christou, Nat. Catal., 2018, $1,48$.

138 Q. Lan, Z.-M. Zhang, C. Qin, X.-L. Wang, Y.-G. Li, H.-Q. Tan and E.-B. Wang, Chem. - Eur. J., 2016, 22, 15513.

139 J. Soriano-López, D. G. Musaev, C. L. Hill, J. R. GalánMascarós, J. J. Carbó and J. M. Poblet, J. Catal., 2017, 350, 56.

140 X.-F. Su, W. Guan, L.-K. Yan and Z.-M. Su, J. Catal., 2020, 381, 402.

141 R. Kaushik, R. Sakla, D. Amilan Jose and A. Ghosh, New J. Chem., 2020, 44, 3764.

142 H. Lv, Y. V. Geletii, C. Zhao, J. W. Vickers, G. Zhu, Z. Luo, J. Song, T. Lian, D. G. Musaev and C. L. Hill, Chem. Soc. Rev., 2012, 41, 7572.

143 S. M. Lauinger, Q. Yin, Y. V. Geletii and C. L. Hill, in Advances in Inorganic Chemistry Polyoxometalate Chemistry, ed. R. van Eldik and L. Cronin, Academic Press, 2017, p. 117.

144 B. Chakraborty, G. Gan-Or, M. Raula, E. Gadot and I. A. Weinstock, Nat. Commun., 2018, 9, 4896.

145 S. Bae, J.-E. Jang, H.-W. Lee and J. Ryu, Eur. J. Inorg. Chem., 2019, 2040.

146 G. A. Volpato, M. Marasi, T. Gobbato, F. Valentini, F. Sabuzi, V. Gagliardi, A. Bonetto, A. Marcomini, S. Berardi, V. Conte, M. Bonchio, S. Caramori, P. Galloni and A. Sartorel, Chem. Commun., 2020, 56, 2248.

147 Q. Yin, J. M. Tan, C. Besson, Y. V. Geletii, D. G. Musaev, A. E. Kuznetsov, Z. Luo, K. I. Hardcastle and C. L. Hill, Science, 2010, 328, 342.

148 J. J. Stracke and R. G. Finke, J. Am. Chem. Soc., 2011, 133, 14872. 
149 J. J. Stracke and R. G. Finke, ACS Catal., 2013, 3, 1209.

150 J. J. Stracke and R. G. Finke, ACS Catal., 2014, 4, 79.

151 J. W. Vickers, H. Lv, J. M. Sumliner, G. Zhu, Z. Luo, D. G. Musaev, Y. V. Geletii and C. L. Hill, J. Am. Chem. Soc., 2013, 135, 14110.

152 H. I. Buvailo, V. G. Makhankova, V. N. Kokozay, I. V. Omelchenko, S. V. Shishkina, J. Jezierska, M. V. Pavliuk and S. I. Shylin, Inorg. Chem. Front., 2019, 6, 1813.

153 L. Yu, Y. Ding and M. Zheng, Appl. Catal., B, 2017, 209, 45.

154 M. Navarro, M. Li, H. Müller-Bunz, S. Bernhard and M. Albrecht, Chem. - Eur. J., 2016, 22, 6740.

155 M. D. Bairagya, R. J. Bujol and N. Elgrishi, Chem. - Eur. J., 2019, 26, 3991.

156 B. Das, A. Thapper, S. Ott and S. B. Colbran, Sustainable Energy Fuels, 2019, 3, 2159.

157 D. P. Hickey, C. Sandford, Z. Rhodes, T. Gensch, L. R. Fries, M. S. Sigman and S. D. Minteer, J. Am. Chem. Soc., 2019, 141, 1382.

158 E. A. Mohamed, Z. N. Zahran and Y. Naruta, J. Catal., 2017, 352, 293.

159 X. Zhou, T. Zhang, C. W. Abney, Z. Li and W. Lin, ACS Appl. Mater. Interfaces, 2014, 6, 18475.

160 P. Garrido-Barros, C. Gimbert-Suriñach, D. Moonshiram, A. Picón, P. Monge, V. S. Batista and A. Llobet, J. Am. Chem. Soc., 2017, 139, 12907.

161 B. Liu, J. Li, H.-L. Wu, W.-Q. Liu, X. Jiang, Z.-J. Li, B. Chen, C.-H. Tung and L.-Z. Wu, ACS Appl. Mater. Interfaces, 2016, 8, 18577.

162 Y. Wang, F. Li, X. Zhou, F. Yu, J. Du, L. Bai and L. Sun, Angew. Chem., Int. Ed., 2017, 56, 6911.

163 S. M. Lauinger, J. M. Sumliner, Q. Yin, Z. Xu, G. Liang, E. N. Glass, T. Lian and C. L. Hill, Chem. Mater., 2015, 27, 5886.

164 S. M. Lauinger, B. D. Piercy, W. Li, Q. Yin, D. L. CollinsWildman, E. N. Glass, M. D. Losego, D. Wang, Y. V. Geletii and C. L. Hill, ACS Appl. Mater. Interfaces, 2017, 9, 35048.

165 B.-T. Chen, N. Morlanés, E. Adogla, K. Takanabe and V. O. Rodionov, ACS Catal., 2016, 6, 4647.

166 G. Paille, M. Gomez-Mingot, C. Roch-Marchal, B. LassalleKaiser, P. Mialane, M. Fontecave, C. Mellot-Draznieks and A. Dolbecq, J. Am. Chem. Soc., 2018, 140, 3613.

167 W. Salomon, F.-J. Yazigi, C. Roch-Marchal, P. Mialane, P. Horcajada, C. Serre, M. Haouas, F. Taulelle and A. Dolbecq, Dalton Trans., 2014, 43, 12698.

168 X.-J. Kong, Z. Lin, Z.-M. Zhang, T. Zhang and W. Lin, Angew. Chem., 2016, 128, 6521.

169 J. Wang, J. Liu, B. Zhang, H. Wan, Z. Li, X. Ji, K. Xu, C. Chen, D. Zha, L. Miao and J. Jiang, Nano Energy, 2017, 42, 98.

170 L. Zhuang, L. Ge, Y. Yang, M. Li, Y. Jia, X. Yao and Z. Zhu, Adv. Mater., 2017, 29, 1606793.

171 W. Wan, S. Wei, J. Li, C. A. Triana, Y. Zhou and G. R. Patzke, J. Mater. Chem. A, 2019, 7, 15145.

172 Y. Zhao, C. K. Mavrokefalos, P. Zhang, R. Erni, J. Li, C. A. Triana and G. R. Patzke, Chem. Mater., 2020, 32, 1371.

173 J. Song, C. Wei, Z.-F. Huang, C. Liu, L. Zeng, X. Wang and Z. J. Xu, Chem. Soc. Rev., 2020, 49, 2196.
174 C. Feng, M. B. Faheem, J. Fu, Y. Xiao, C. Li and Y. Li, ACS Catal., 2020, 10, 4019.

175 J. Y. C. Chen, L. Dang, H. Liang, W. Bi, J. B. Gerken, S. Jin, E. E. Alp and S. S. Stahl, J. Am. Chem. Soc., 2015, 137, 15090.

176 L. Francàs, S. Corby, S. Selim, D. Lee, C. A. Mesa, R. Godin, E. Pastor, I. E. L. Stephens, K.-S. Choi and J. R. Durrant, Nat. Commun., 2019, 10, 5208.

177 S. Lee, L. Bai and X. Hu, Angew. Chem., Int. Ed., 2020, 59, 8072.

178 M. M. Najafpour, G. Renger, M. Hołyńska, A. N. Moghaddam, E.-M. Aro, R. Carpentier, H. Nishihara, J. J. Eaton-Rye, J.-R. Shen and S. I. Allakhverdiev, Chem. Rev., 2016, 116, 2886.

179 M. W. Kanan, J. Yano, Y. Surendranath, M. Dincă, V. K. Yachandra and D. G. Nocera, J. Am. Chem. Soc., 2010, 132, 13692.

180 J. B. Gerken, J. G. McAlpin, J. Y. C. Chen, M. L. Rigsby, W. H. Casey, R. D. Britt and S. S. Stahl, J. Am. Chem. Soc., 2011, 133, 14431.

181 S. R. Pendlebury, M. Barroso, A. J. Cowan, K. Sivula, J. Tang, M. Grätzel, D. Klug and J. R. Durrant, Chem. Commun., 2011, 47, 716.

182 C. Y. Cummings, F. Marken, L. M. Peter, A. A. Tahir and K. G. U. Wijayantha, Chem. Commun., 2012, 48, 2027.

183 B. Klahr, S. Gimenez, F. Fabregat-Santiago, J. Bisquert and T. W. Hamann, Energy Environ. Sci., 2012, 5, 7626.

184 M. Barroso, S. R. Pendlebury, A. J. Cowan and J. R. Durrant, Chem. Sci., 2013, 4, 2724.

185 K. M. H. Young, B. M. Klahr, O. Zandi and T. W. Hamann, Catal. Sci. Technol., 2013, 3, 1660.

186 H. S. Ahn and A. J. Bard, J. Am. Chem. Soc., 2015, 137, 612. 187 J. Rodríguez-López, M. A. Alpuche-Avilés and A. J. Bard, J. Am. Chem. Soc., 2008, 130, 16985.

188 C. Costentin, T. R. Porter and J.-M. Savéant, J. Am. Chem. Soc., 2016, 138, 5615.

189 R. Nakamura and Y. Nakato, J. Am. Chem. Soc., 2004, 126, 1290.

190 M. Zhang, M. de Respinis and H. Frei, Nat. Chem., 2014, 6, 362. 191 H. H. Pham, M.-J. Cheng, H. Frei and L.-W. Wang, ACS Catal., 2016, 6, 5610.

192 S. Koroidov, M. F. Anderlund, S. Styring, A. Thapper and J. Messinger, Energy Environ. Sci., 2015, 8, 2492.

193 A. M. Ullman, C. N. Brodsky, N. Li, S.-L. Zheng and D. G. Nocera, J. Am. Chem. Soc., 2016, 138, 4229.

194 J. Fester, M. García-Melchor, A. S. Walton, M. Bajdich, Z. Li, L. Lammich, A. Vojvodic and J. V. Lauritsen, Nat. Commun., 2017, 8, 14169.

195 G. S. Hutchings, Y. Zhang, J. Li, B. T. Yonemoto, X. Zhou, K. Zhu and F. Jiao, J. Am. Chem. Soc., 2015, 137, 4223.

196 H.-Y. Wang, S.-F. Hung, H.-Y. Chen, T.-S. Chan, H. M. Chen and B. Liu, J. Am. Chem. Soc., 2016, 138, 36.

197 H.-Y. Wang, S.-F. Hung, Y.-Y. Hsu, L. Zhang, J. Miao, T.-S. Chan, Q. Xiong and B. Liu, J. Phys. Chem. Lett., 2016, 7, 4847.

198 S. W. Lee, C. Carlton, M. Risch, Y. Surendranath, S. Chen, S. Furutsuki, A. Yamada, D. G. Nocera and Y. Shao-Horn, J. Am. Chem. Soc., 2012, 134, 16959. 
199 D. González-Flores, I. Sánchez, I. Zaharieva, K. Klingan, J. Heidkamp, P. Chernev, P. W. Menezes, M. Driess, H. Dau and M. L. Montero, Angew. Chem., Int. Ed., 2015, 54, 2472.

200 N. Weidler, S. Paulus, J. Schuch, J. Klett, S. Hoch, P. Stenner, A. Maljusch, J. Brötz, C. Wittich, B. Kaiser and W. Jaegermann, Phys. Chem. Chem. Phys., 2016, 18, 10708.

201 K. Sivula, F. Le Formal and M. Grätzel, ChemSusChem, 2011, 4, 432.

202 O. Zandi and T. W. Hamann, Nat. Chem., 2016, 8, 778.

203 S. Haschke, M. Mader, S. Schlicht, A. M. Roberts, A. M. Angeles-Boza, J. A. C. Barth and J. Bachmann, Nat. Commun., 2018, 9, 4565.

204 F. Le Formal, E. Pastor, S. D. Tilley, C. A. Mesa, S. R. Pendlebury, M. Grätzel and J. R. Durrant, J. Am. Chem. Soc., 2015, 137, 6629.

205 C. A. Mesa, L. Francàs, K. R. Yang, P. Garrido-Barros, E. Pastor, Y. Ma, A. Kafizas, T. E. Rosser, M. T. Mayer, E. Reisner, M. Grätzel, V. S. Batista and J. R. Durrant, Nat. Chem., 2020, 12, 82.

206 Y. Zhang, H. Zhang, A. Liu, C. Chen, W. Song and J. Zhao, J. Am. Chem. Soc., 2018, 140, 3264.

207 Y. Zhang, H. Zhang, H. Ji, W. Ma, C. Chen and J. Zhao, J. Am. Chem. Soc., 2016, 138, 2705.

208 D. M. Herlihy, M. M. Waegele, X. Chen, C. D. Pemmaraju, D. Prendergast and T. Cuk, Nat. Chem., 2016, 8, 549.

209 X. Chen, D. J. Aschaffenburg and T. Cuk, Nat. Catal., 2019, 2, 820 .

210 M. Zhang and H. Frei, Catal. Lett., 2015, 145, 420.

211 I. C. Man, H.-Y. Su, F. Calle-Vallejo, H. A. Hansen, J. I. Martínez, N. G. Inoglu, J. Kitchin, T. F. Jaramillo, J. K. Nørskov and J. Rossmeisl, ChemCatChem, 2011, 3, 1159.

212 M. Kuang, P. Han, L. Huang, N. Cao, L. Qian and G. Zheng, Adv. Funct. Mater., 2018, 28, 1804886.

213 M. Görlin, P. Chernev, J. Ferreira de Araújo, T. Reier, S. Dresp, B. Paul, R. Krähnert, H. Dau and P. Strasser, J. Am. Chem. Soc., 2016, 138, 5603.

214 Z. Li, W. Niu, L. Zhou and Y. Yang, ACS Energy Lett., 2018, 3, 892.

215 H. Hajiyani and R. Pentcheva, ACS Catal., 2018, 8, 11773.

216 Y. Zhou, S. Sun, J. Song, S. Xi, B. Chen, Y. Du, A. C. Fisher, F. Cheng, X. Wang, H. Zhang and Z. J. Xu, Adv. Mater., 2018, 30, 1802912.

217 M. Görlin, J. Ferreira de Araújo, H. Schmies, D. Bernsmeier, S. Dresp, M. Gliech, Z. Jusys, P. Chernev, R. Kraehnert, H. Dau and P. Strasser, J. Am. Chem. Soc., 2017, 139, 2070.

218 Y. Hu, Z. Wang, W. Liu, L. Xu, M. Guan, Y. Huang, Y. Zhao, J. Bao and H.-M. Li, ACS Sustainable Chem. Eng., 2019, 7, 16828.

219 K. Fan, H. Chen, Y. Ji, H. Huang, P. M. Claesson, Q. Daniel, B. Philippe, H. Rensmo, F. Li, Y. Luo and L. Sun, Nat. Commun., 2016, 7, 11981.

220 J. Bao, Z. Wang, J. Xie, L. Xu, F. Lei, M. Guan, Y. Zhao, Y. Huang and H. Li, Chem. Commun., 2019, 55, 3521.

221 Y. Wang, Y. Sun, F. Yan, C. Zhu, P. Gao, X. Zhang and Y. Chen, J. Mater. Chem. A, 2018, 6, 8479.
222 S. Sun, Y. Sun, Y. Zhou, J. Shen, D. Mandler, R. Neumann and Z. J. Xu, Chem. Mater., 2019, 31, 8106.

223 T. Tian, H. Gao, X. Zhou, L. Zheng, J. Wu, K. Li and Y. Ding, ACS Energy Lett., 2018, 3, 2150.

224 Z.-F. Huang, J. Song, Y. Du, S. Xi, S. Dou, J. M. V. Nsanzimana, C. Wang, Z. J. Xu and X. Wang, Nat. Energy, 2019, 4, 329.

225 T. Wu, S. Sun, J. Song, S. Xi, Y. Du, B. Chen, W. A. Sasangka, H. Liao, C. L. Gan, G. G. Scherer, L. Zeng, H. Wang, H. Li, A. Grimaud and Z. J. Xu, Nat. Catal., 2019, 2, 763.

226 D.-H. Seo, J. Lee, A. Urban, R. Malik, S. Kang and G. Ceder, Nat. Chem., 2016, 8, 692.

227 G. Assat and J.-M. Tarascon, Nat. Energy, 2018, 3, 373.

228 J. Lee, D. A. Kitchaev, D.-H. Kwon, C.-W. Lee, J. K. Papp, Y.S. Liu, Z. Lun, R. J. Clément, T. Shi, B. D. McCloskey, J. Guo, M. Balasubramanian and G. Ceder, Nature, 2018, 556, 185.

229 J. Song, C. Wei, Z.-F. Huang, C. Liu, L. Zeng, X. Wang and Z. J. Xu, Chem. Soc. Rev., 2020, 49, 2196.

230 M.-Q. Yang, J. Wang, H. Wu and G. W. Ho, Small, 2018, 14, 1703323.

231 T. Ling, D.-Y. Yan, Y. Jiao, H. Wang, Y. Zheng, X. Zheng, J. Mao, X.-W. Du, Z. Hu, M. Jaroniec and S.-Z. Qiao, Nat. Commun., 2016, 7, 12876.

232 L. Xu, Q. Jiang, Z. Xiao, X. Li, J. Huo, S. Wang and L. Dai, Angew. Chem., Int. Ed., 2016, 55, 5277.

233 X. Zhang, Y. Zhao, Y. Zhao, R. Shi, G. I. N. Waterhouse and T. Zhang, Adv. Energy Mater., 2019, 9, 1900881.

234 R. Zhang, Y.-C. Zhang, L. Pan, G.-Q. Shen, N. Mahmood, Y.-H. Ma, Y. Shi, W. Jia, L. Wang, X. Zhang, W. Xu and J.-J. Zou, ACS Catal., 2018, 8, 3803.

235 D. Ji, L. Fan, L. Tao, Y. Sun, M. Li, G. Yang, T. Q. Tran, S. Ramakrishna and S. Guo, Angew. Chem., Int. Ed., 2019, 58, 13840.

236 Z. Li, Y. Zhang, Y. Feng, C.-Q. Cheng, K.-W. Qiu, C.-K. Dong, H. Liu and X.-W. Du, Adv. Funct. Mater., 2019, 29, 1903444.

237 Y. Wang, Y. Zhang, Z. Liu, C. Xie, S. Feng, D. Liu, M. Shao and S. Wang, Angew. Chem., Int. Ed., 2017, 56, 5867.

238 Q. He, Y. Wan, H. Jiang, Z. Pan, C. Wu, M. Wang, X. Wu, B. Ye, P. M. Ajayan and L. Song, ACS Energy Lett., 2018, 3, 1373.

239 Y. Zhao, W. Wan, Y. Chen, R. Erni, C. A. Triana, J. Li, C. K. Mavrokefalos, Y. Zhou and G. R. Patzke, Adv. Energy Mater., 2020, 10, 2002228.

240 X. Du, J. Huang, J. Zhang, Y. Yan, C. Wu, Y. Hu, C. Yan, T. Lei, W. Chen, C. Fan and J. Xiong, Angew. Chem., Int. Ed., 2019, 58, 4484.

241 H. Li, C. Tsai, A. L. Koh, L. Cai, A. W. Contryman, A. H. Fragapane, J. Zhao, H. S. Han, H. C. Manoharan, F. Abild-Pedersen, J. K. Nørskov and X. Zheng, Nat. Mater., 2016, 15, 48.

242 W. Cheng, X. Zhao, H. Su, F. Tang, W. Che, H. Zhang and Q. Liu, Nat. Energy, 2019, 4, 115.

243 D. Zhou, S. Wang, Y. Jia, X. Xiong, H. Yang, S. Liu, J. Tang, J. Zhang, D. Liu, L. Zheng, Y. Kuang, X. Sun and B. Liu, Angew. Chem., Int. Ed., 2019, 58, 736. 
244 L. Wang, K. A. Stoerzinger, L. Chang, X. Yin, Y. Li, C. S. Tang, E. Jia, M. E. Bowden, Z. Yang, A. Abdelsamie, L. You, R. Guo, J. Chen, A. Rusydi, J. Wang, S. A. Chambers and Y. Du, ACS Appl. Mater. Interfaces, 2019, 11, 12941.

245 L. Wang, Z. Zeng, W. Gao, T. Maxson, D. Raciti, M. Giroux, X. Pan, C. Wang and J. Greeley, Science, 2019, 363, 870.

246 T. Ling, D.-Y. Yan, H. Wang, Y. Jiao, Z. Hu, Y. Zheng, L. Zheng, J. Mao, H. Liu, X.-W. Du, M. Jaroniec and S.Z. Qiao, Nat. Commun., 2017, 8, 1509.

247 J. R. Petrie, V. R. Cooper, J. W. Freeland, T. L. Meyer, Z. Zhang, D. A. Lutterman and H. N. Lee, J. Am. Chem. Soc., 2016, 138, 2488.

248 Z. Chen, C. X. Kronawitter and B. E. Koel, Phys. Chem. Chem. Phys., 2015, 17, 29387.

249 R. Gao, J. Zhu, X. Xiao, Z. Hu, J. Liu and X. Liu, J. Phys. Chem. C, 2015, 119, 4516.

250 L. Liu, Z. Jiang, L. Fang, H. Xu, H. Zhang, X. Gu and Y. Wang, ACS Appl. Mater. Interfaces, 2017, 9, 27736.

251 X. Han, G. He, Y. He, J. Zhang, X. Zheng, L. Li, C. Zhong, W. Hu, Y. Deng and T.-Y. Ma, Adv. Energy Mater., 2018, 8, 1702222.

252 X. Zhou, Z. Liu, Y. Wang and Y. Ding, Appl. Catal., B, 2018, 237, 74 .

253 A. Govind Rajan, J. M. P. Martirez and E. A. Carter, J. Am. Chem. Soc., 2020, 142, 3600.

254 J. Deng, D. Deng and X. Bao, Adv. Mater., 2017, 29, 1606967.

255 Q. Lu, J. Yu, X. Zou, K. Liao, P. Tan, W. Zhou, M. Ni and Z. Shao, Adv. Funct. Mater., 2019, 29, 1904481.

256 H. Khani, N. S. Grundish, D. O. Wipf and J. B. Goodenough, Adv. Energy Mater., 2020, 10, 1903215.

257 X. Cui, P. Ren, D. Deng, J. Deng and X. Bao, Energy Environ. Sci., 2016, 9, 123.

258 X. Han, W. Zhang, X. Ma, C. Zhong, N. Zhao, W. Hu and Y. Deng, Adv. Mater., 2019, 31, 1808281.

259 J. Zhang, B. Qian, S. Sun, S. Tao, W. Chu, D. Wu and L. Song, Small, 2019, 15, 1904260.

260 L. Zhang, J. Xiao, H. Wang and M. Shao, ACS Catal., 2017, 7, 7855.

261 Y. Li, X. Du, J. Huang, C. Wu, Y. Sun, G. Zou, C. Yang and J. Xiong, Small, 2019, 15, e1901980.

262 M. M. Najafpour, M. Abbasi Isaloo, M. Abasi and M. Hołyńska, New J. Chem., 2014, 38, 852.

263 J. O’M. Bockris and T. Otagawa, J. Electrochem. Soc., 2019, 131, 290.

264 W. G. Hardin, J. T. Mefford, D. A. Slanac, B. B. Patel, X. Wang, S. Dai, X. Zhao, R. S. Ruoff, K. P. Johnston and K. J. Stevenson, Chem. Mater., 2014, 26, 3368.

265 W. T. Hong, M. Risch, K. A. Stoerzinger, A. Grimaud, J. Suntivich and Y. Shao-Horn, Energy Environ. Sci., 2015, 8, 1404.

266 R. A. Rincón, E. Ventosa, F. Tietz, J. Masa, S. Seisel, V. Kuznetsov and W. Schuhmann, ChemPhysChem, 2014, 15, 2810.

267 W. Zhou, M. Zhao, F. Liang, S. C. Smith and Z. Zhu, Mater. Horiz., 2015, 2, 495.
268 C. Lawley, M. Nachtegaal, J. Stahn, V. Roddatis, M. Döbeli, T. J. Schmidt, D. Pergolesi and T. Lippert, Nat. Commun., 2020, 11, 1728.

269 B.-J. Kim, E. Fabbri, D. F. Abbott, X. Cheng, A. H. Clark, M. Nachtegaal, M. Borlaf, I. E. Castelli, T. Graule and T. J. Schmidt, J. Am. Chem. Soc., 2019, 141, 5231.

270 S. H. Chang, N. Danilovic, K.-C. Chang, R. Subbaraman, A. P. Paulikas, D. D. Fong, M. J. Highland, P. M. Baldo, V. R. Stamenkovic, J. W. Freeland, J. A. Eastman and N. M. Markovic, Nat. Commun., 2014, 5, 1.

271 X. Rong, J. Parolin and A. M. Kolpak, ACS Catal., 2016, 6, 1153.

272 B. Han, M. Risch, Y.-L. Lee, C. Ling, H. Jia and Y. ShaoHorn, Phys. Chem. Chem. Phys., 2015, 17, 22576.

273 K. J. May, C. E. Carlton, K. A. Stoerzinger, M. Risch, J. Suntivich, Y.-L. Lee, A. Grimaud and Y. Shao-Horn, J. Phys. Chem. Lett., 2012, 3, 3264.

274 E. Fabbri, M. Nachtegaal, T. Binninger, X. Cheng, B.-J. Kim, J. Durst, F. Bozza, T. Graule, R. Schäublin, L. Wiles, M. Pertoso, N. Danilovic, K. E. Ayers and T. J. Schmidt, Nat. Mater., 2017, 16, 925.

275 A. I. Nguyen, K. M. van Allsburg, M. W. Terban, M. Bajdich, J. Oktawiec, J. Amtawong, M. S. Ziegler, J. P. Dombrowski, K. V. Lakshmi, W. S. Drisdell, J. Yano, S. J. L. Billinge and T. D. Tilley, Proc. Natl. Acad. Sci. U. S. A., 2019, 116, 11630.

276 D. Mierwaldt, V. Roddatis, M. Risch, J. Scholz, J. Geppert, M. E. Abrishami and C. Jooss, Adv. Sustainable Syst., 2017, 1, 1700109.

277 S. Heidari, J. P. Singh, H. Feizi, R. Bagheri, K. H. Chae, Z. Song, M. Khatamian and M. M. Najafpour, Sci. Rep., 2019, 9, 7749.

278 S. Heidari, M. M. Najafpour, M. Hołyńska, J. P. Singh, K. H. Chae and M. Khatamian, Dalton Trans., 2018, 47, 1557.

279 K. Fan, H. Zou, Y. Lu, H. Chen, F. Li, J. Liu, L. Sun, L. Tong, M. F. Toney, M. Sui and J. Yu, ACS Nano, 2018, 12, 12369.

280 Y.-J. Tang, Y. Wang and K. Zhou, J. Mater. Chem. A, 2020, 8, 7925.

281 X. Zhang, Y.-S. Chen, P. V. Kamat and S. Ptasinska, J. Phys. Chem. C, 2018, 122, 13894.

282 D. Friebel, M. W. Louie, M. Bajdich, K. E. Sanwald, Y. Cai, A. M. Wise, M.-J. Cheng, D. Sokaras, T.-C. Weng, R. AlonsoMori, R. C. Davis, J. R. Bargar, J. K. Nørskov, A. Nilsson and A. T. Bell, J. Am. Chem. Soc., 2015, 137, 1305.

283 T. Morikawa, S. Gul, Y. F. Nishimura, T. M. Suzuki and J. Yano, Chem. Commun., 2020, 56, 5158.

284 R. D. L. Smith, C. Pasquini, S. Loos, P. Chernev, K. Klingan, P. Kubella, M. R. Mohammadi, D. González-Flores and H. Dau, Energy Environ. Sci., 2018, 11, 2476.

285 D. González-Flores, K. Klingan, P. Chernev, S. Loos, M. R. Mohammadi, C. Pasquini, P. Kubella, I. Zaharieva, R. D. L. Smith and H. Dau, Sustainable Energy Fuels, 2018, 2, 1986.

286 N. Ortiz Peña, D. Ihiawakrim, M. Han, B. Lassalle-Kaiser, S. Carenco, C. Sanchez, C. Laberty-Robert, D. Portehault and O. Ersen, ACS Nano, 2019, 13, 11372. 
287 S. Klaus, Y. Cai, M. W. Louie, L. Trotochaud and A. T. Bell, J. Phys. Chem. C, 2015, 119, 7243.

288 B. M. Hunter, N. B. Thompson, A. M. Müller, G. R. Rossman, M. G. Hill, J. R. Winkler and H. B. Gray, Joule, 2018, 2, 747.

289 L. Trotochaud, S. L. Young, J. K. Ranney and S. W. Boettcher, J. Am. Chem. Soc., 2014, 136, 6744.

290 Z. Zand, P. Salimi, M. R. Mohammadi, R. Bagheri, P. Chernev, Z. Song, H. Dau, M. Görlin and M. M. Najafpour, ACS Sustainable Chem. Eng., 2019, 7, 17252.

291 S. Back, K. Tran and Z. W. Ulissi, ACS Catal., 2019, 9, 7651.

292 A. Nandy, J. Zhu, J. P. Janet, C. Duan, R. B. Getman and H. J. Kulik, ACS Catal., 2019, 9, 8243.

293 R. Palkovits and S. Palkovits, ACS Catal., 2019, 9, 8383.

294 M. S. Burke, M. G. Kast, L. Trotochaud, A. M. Smith and S. W. Boettcher, J. Am. Chem. Soc., 2015, 137, 3638.

295 P. W. Menezes, A. Indra, D. González-Flores, N. R. Sahraie, I. Zaharieva, M. Schwarze, P. Strasser, H. Dau and M. Driess, ACS Catal., 2015, 5, 2017.

296 R. D. L. Smith, C. Pasquini, S. Loos, P. Chernev, K. Klingan, P. Kubella, M. R. Mohammadi, D. Gonzalez-Flores and H. Dau, Nat. Commun., 2017, 8, 2022.

297 M. Risch, A. Grimaud, K. J. May, K. A. Stoerzinger, T. J. Chen, A. N. Mansour and Y. Shao-Horn, J. Phys. Chem. C, 2013, 117, 8628.

298 A. Indra, P. W. Menezes, C. Das, C. Göbel, M. Tallarida, D. Schmeißer and M. Driess, J. Mater. Chem. A, 2017, 5, 5171.

299 R. Zhang, Z. Wang, S. Hao, R. Ge, X. Ren, F. Qu, G. Du, A. M. Asiri, B. Zheng and X. Sun, ACS Sustainable Chem. Eng., 2017, 5, 8518.

300 Z. Chen, Z. Duan, Z. Wang, X. Liu, L. Gu, F. Zhang, M. Dupuis and C. Li, ChemCatChem, 2017, 9, 3641.

301 J. S. Kim, I. Park, E.-S. Jeong, K. Jin, W. M. Seong, G. Yoon, H. Kim, B. Kim, K. T. Nam and K. Kang, Adv. Mater., 2017, 29, 1606893.

302 L. Liardet and X. Hu, ACS Catal., 2018, 8, 644.

303 X. Wang, W. Ma, C. Ding, Z. Xu, H. Wang, X. Zong and C. Li, ACS Catal., 2018, 8, 9926.

304 K. Klingan, F. Ringleb, I. Zaharieva, J. Heidkamp, P. Chernev, D. Gonzalez-Flores, M. Risch, A. Fischer and H. Dau, ChemSusChem, 2014, 7, 1301.

305 P. Du, O. Kokhan, K. W. Chapman, P. J. Chupas and D. M. Tiede, J. Am. Chem. Soc., 2012, 134, 11096.

306 J.-H. Xu, L.-Y. Guo, H.-F. Su, X. Gao, X.-F. Wu, W.-G. Wang, C.-H. Tung and D. Sun, Inorg. Chem., 2017, 56, 1591.

307 D. Y. Shopov, L. S. Sharninghausen, S. B. Sinha, B. Q. Mercado, D. Balcells, G. W. Brudvig and R. H. Crabtree, Inorg. Chem., 2018, 57, 5684.

308 M. Schilling and S. Luber, Front. Chem., 2018, 6, 100.

309 I. D. Young, M. Ibrahim, R. Chatterjee, S. Gul, F. Fuller, S. Koroidov, A. S. Brewster, R. Tran, R. Alonso-Mori, T. Kroll, T. Michels-Clark, H. Laksmono, R. G. Sierra, C. A. Stan, R. Hussein, M. Zhang, L. Douthit, M. Kubin, C. de Lichtenberg, P. Long Vo, H. Nilsson, M. H. Cheah, D. Shevela, C. Saracini, M. A. Bean, I. Seuffert, D. Sokaras,
T.-C. Weng, E. Pastor, C. Weninger, T. Fransson, L. Lassalle, P. Bräuer, P. Aller, P. T. Docker, B. Andi, A. M. Orville, J. M. Glownia, S. Nelson, M. Sikorski, D. Zhu, M. S. Hunter, T. J. Lane, A. Aquila, J. E. Koglin, J. Robinson, M. Liang, S. Boutet, A. Y. Lyubimov, M. Uervirojnangkoorn, N. W. Moriarty, D. Liebschner, P. V. Afonine, D. G. Waterman, G. Evans, P. Wernet, H. Dobbek, W. I. Weis, A. T. Brunger, P. H. Zwart, P. D. Adams, A. Zouni, J. Messinger, U. Bergmann, N. K. Sauter, J. Kern, V. K. Yachandra and J. Yano, Nature, 2016, 540, 453.

310 D. J. Vinyard and G. W. Brudvig, Annu. Rev. Phys. Chem., 2017, 68, 101.

311 D. A. Lutterman, Y. Surendranath and D. G. Nocera, J. Am. Chem. Soc., 2009, 131, 3838.

312 Y. Zhao, J. Lin, Y. Liu, B. Ma, Y. Ding and M. Chen, Chem. Commun., 2015, 51, 17309.

313 P. F. Smith, C. Kaplan, J. E. Sheats, D. M. Robinson, N. S. McCool, N. Mezle and G. C. Dismukes, Inorg. Chem., 2014, 53, 2113.

314 F. Song, K. Al-Ameed, M. Schilling, T. Fox, S. Luber and G. R. Patzke, J. Am. Chem. Soc., 2019, 141, 8846.

315 Z. W. Seh, J. Kibsgaard, C. F. Dickens, I. Chorkendorff, J. K. Nørskov and T. F. Jaramillo, Science, 2017, 355, 6321. 316 J. Greeley, Annu. Rev. Chem. Biomol. Eng., 2016, 7, 605.

317 M. Busch, A. Fabrizio, S. Luber, J. Hutter and C. Corminboeuf, J. Phys. Chem. C, 2018, 122, 12404.

318 J. K. Nørskov, J. Rossmeisl, A. Logadottir, L. Lindqvist, J. R. Kitchin, T. Bligaard and H. Jónsson, J. Phys. Chem. B, 2004, 108, 17886.

319 J. Rossmeisl, E. Skúlason, M. E. Björketun, V. Tripkovic and J. K. Nørskov, Chem. Phys. Lett., 2008, 466, 68.

320 M. Busch, Curr. Opin. Electrochem., 2018, 9, 278.

321 J. Hessels, R. J. Detz, M. T. M. Koper and J. N. H. Reek, Chem. - Eur. J., 2017, 23, 16413.

322 K. Fan, Y. Ji, H. Zou, J. Zhang, B. Zhu, H. Chen, Q. Daniel, Y. Luo, J. Yu and L. Sun, Angew. Chem., Int. Ed., 2017, 56, 3289.

323 Z.-F. Huang, J. Song, S. Dou, X. Li, J. Wang and X. Wang, Matter, 2019, 1, 1494.

324 B. Zhang, X. Zheng, O. Voznyy, R. Comin, M. Bajdich, M. García-Melchor, L. Han, J. Xu, M. Liu, L. Zheng, F. P. García de Arquer, C. T. Dinh, F. Fan, M. Yuan, E. Yassitepe, N. Chen, T. Regier, P. Liu, Y. Li, P. de Luna, A. Janmohamed, H. L. Xin, H. Yang, A. Vojvodic and E. H. Sargent, Science, 2016, 352, 333.

325 M. J. Craig, G. Coulter, E. Dolan, J. Soriano-López, E. Mates-Torres, W. Schmitt and M. García-Melchor, Nat. Commun., 2019, 10, 4993.

326 B. Schwarz, J. Forster, M. K. Goetz, D. Yücel, C. Berger, T. Jacob and C. Streb, Angew. Chem., Int. Ed., 2016, 55, 6329.

327 R. Al-Oweini, A. Sartorel, B. S. Bassil, M. Natali, S. Berardi, F. Scandola, U. Kortz and M. Bonchio, Angew. Chem., Int. Ed., 2014, 53, 11182.

328 M. L. Pegis, C. F. Wise, B. Koronkiewicz and J. M. Mayer, J. Am. Chem. Soc., 2017, 139, 11000. 
329 Y. Chen, S. Ji, C. Chen, Q. Peng, D. Wang and Y. Li, Joule, 2018, 2, 1242.

330 B. Limburg, E. Bouwman and S. Bonnet, Coord. Chem. Rev., 2012, 256, 1451.

331 L. Zhang, Y. Jia, G. Gao, X. Yan, N. Chen, J. Chen, M. T. Soo, B. Wood, D. Yang, A. Du and X. Yao, Chem, 2018, 4, 285.

332 H. Fei, J. Dong, Y. Feng, C. S. Allen, C. Wan, B. Volosskiy, M. Li, Z. Zhao, Y. Wang, H. Sun, P. An, W. Chen, Z. Guo, C. Lee, D. Chen, I. Shakir, M. Liu, T. Hu, Y. Li, A. I. Kirkland, X. Duan and Y. Huang, Nat. Catal., 2018, 1, 63.

333 Q. Li, W. Chen, H. Xiao, Y. Gong, Z. Li, L. Zheng, X. Zheng, W. Yan, W.-C. Cheong, R. Shen, N. Fu, L. Gu, Z. Zhuang, C. Chen, D. Wang, Q. Peng, J. Li and Y. Li, Adv. Mater., 2018, 30, 1800588.

334 J. Guan, Z. Duan, F. Zhang, S. D. Kelly, R. Si, M. Dupuis, Q. Huang, J. Q. Chen, C. Tang and C. Li, Nat. Catal., 2018, 1,870 .

335 P. Chen, T. Zhou, L. Xing, K. Xu, Y. Tong, H. Xie, L. Zhang, W. Yan, W. Chu, C. Wu and Y. Xie, Angew. Chem., Int. Ed., 2017, 56, 610.

336 Y. Zheng, Y. Jiao, Y. Zhu, Q. Cai, A. Vasileff, L. H. Li, Y. Han, Y. Chen and S.-Z. Qiao, J. Am. Chem. Soc., 2017, 139, 3336.

337 Y. Yao, S. Hu, W. Chen, Z.-Q. Huang, W. Wei, T. Yao, R. Liu, K. Zang, X. Wang, G. Wu, W. Yuan, T. Yuan, B. Zhu, W. Liu, Z. Li, D. He, Z. Xue, Y. Wang, X. Zheng, J. Dong, C.-R. Chang, Y. Chen, X. Hong, J. Luo, S. Wei, W.-X. Li, P. Strasser, Y. Wu and Y. Li, Nat. Catal., 2019, 2, 304.

338 W. Liu, L. Cao, W. Cheng, Y. Cao, X. Liu, W. Zhang, X. Mou, L. Jin, X. Zheng, W. Che, Q. Liu, T. Yao and S. Wei, Angew. Chem., Int. Ed., 2017, 56, 9312.

339 J. Zhang, J. Liu, L. Xi, Y. Yu, N. Chen, S. Sun, W. Wang, K. M. Lange and B. Zhang, J. Am. Chem. Soc., 2018, 140, 3876.

340 C. Lin, Y. Zhao, H. Zhang, S. Xie, Y.-F. Li, X. Li, Z. Jiang and Z.-P. Liu, Chem. Sci., 2018, 9, 6803.

341 P. Li, M. Wang, X. Duan, L. Zheng, X. Cheng, Y. Zhang, Y. Kuang, Y. Li, Q. Ma, Z. Feng, W. Liu and X. Sun, Nat. Commun., 2019, 10, 1711.

342 J. Yan, L. Kong, Y. Ji, J. White, Y. Li, J. Zhang, P. An, S. Liu, S.-T. Lee and T. Ma, Nat. Commun., 2019, 10, 2149.

343 Q. Wang, X. Huang, Z. L. Zhao, M. Wang, B. Xiang, J. Li, Z. Feng, H. Xu and M. Gu, J. Am. Chem. Soc., 2020, $142,7425$.

344 Y. Pan, R. Lin, Y. Chen, S. Liu, W. Zhu, X. Cao, W. Chen, K. Wu, W.-C. Cheong, Y. Wang, L. Zheng, J. Luo, Y. Lin, Y. Liu, C. Liu, J. Li, Q. Lu, X. Chen, D. Wang, Q. Peng, C. Chen and Y. Li, J. Am. Chem. Soc., 2018, 140, 4218.

345 S. Zhan and M. S. G. Ahlquist, J. Am. Chem. Soc., 2018, 140, 7498.

346 F. Li, B. Zhang, X. Li, Y. Jiang, L. Chen, Y. Li and L. Sun, Angew. Chem., Int. Ed., 2011, 50, 12276.

347 T. Xue, B. Peng, M. Xue, X. Zhong, C.-Y. Chiu, S. Yang, Y. Qu, L. Ruan, S. Jiang, S. Dubin, R. B. Kaner, J. I. Zink,
M. E. Meyerhoff, X. Duan and Y. Huang, Nat. Commun., 2014, 5, 3200.

348 F. Li, H. Yang, W. Li and L. Sun, Joule, 2018, 2, 36.

349 N. Kuwamura, Y. Kurioka, N. Yoshinari and T. Konno, Chem. Commun., 2018, 54, 10766.

350 P. Christopher, ACS Energy Lett., 2019, 4, 2249.

351 H. Xu, D. Cheng, D. Cao and X. C. Zeng, Nat. Catal., 2018, 1, 339.

352 G. Gao, S. Bottle and A. Du, Catal. Sci. Technol., 2018, 8, 996.

353 Y. Hou, M. Qiu, M. G. Kim, P. Liu, G. Nam, T. Zhang, X. Zhuang, B. Yang, J. Cho, M. Chen, C. Yuan, L. Lei and X. Feng, Nat. Commun., 2019, 10, 1392.

354 Y. Li, Z.-S. Wu, P. Lu, X. Wang, W. Liu, Z. Liu, J. Ma, W. Ren, Z. Jiang and X. Bao, Adv. Sci., 2020, 7, 1903089.

355 J. Zhang, Y. Zhao, C. Chen, Y.-C. Huang, C.-L. Dong, C.-J. Chen, R.-S. Liu, C. Wang, K. Yan, Y. Li and G. Wang, J. Am. Chem. Soc., 2019, 141, 20118.

356 B. Lu, Q. Liu and S. Chen, ACS Catal., 2020, 10, 7584.

357 W. Wan, C. A. Triana, J. Lan, J. Li, C. S. Allen, Y. Zhao, M. Iannuzzi and G. R. Patzke, ACS Nano, 2020, 14, 13279. 358 B. Wurster, D. Grumelli, D. Hötger, R. Gutzler and K. Kern, J. Am. Chem. Soc., 2016, 138, 3623.

359 H. Xiao, H. Shin and W. A. Goddard, Proc. Natl. Acad. Sci. U. S. A., 2018, 115, 5872.

360 J. Wang, L. Gan, W. Zhang, Y. Peng, H. Yu, Q. Yan, X. Xia and X. Wang, Sci. Adv., 2018, 4, 7970.

361 L. Bai, C.-S. Hsu, D. T. L. Alexander, H. M. Chen and X. Hu, J. Am. Chem. Soc., 2019, 141, 14190.

362 S. Fukuzumi, Y.-M. Lee and W. Nam, ChemCatChem, 2018, 10, 1686.

363 Y. Zhao, X. Yan, K. R. Yang, S. Cao, Q. Dong, J. E. Thorne, K. L. Materna, S. Zhu, X. Pan, M. Flytzani-Stephanopoulos, G. W. Brudvig, V. S. Batista and D. Wang, ACS Cent. Sci., 2018, 4, 1166.

364 S. W. Sheehan, J. M. Thomsen, U. Hintermair, R. H. Crabtree, G. W. Brudvig and C. A. Schmuttenmaer, Nat. Commun., 2015, 6, 6469.

365 Z. N. Zahran, Y. Tsubonouchi, E. A. Mohamed and M. Yagi, ChemSusChem, 2019, 12, 1775.

366 Z. Chen, J. J. Concepcion, J. F. Hull, P. G. Hoertz and T. J. Meyer, Dalton Trans., 2010, 39, 6950.

367 G. Pastori, K. Wahab, A. Bucci, G. Bellachioma, C. Zuccaccia, J. Llorca, H. Idriss and A. Macchioni, Chem. - Eur. J., 2016, 22, 13459.

368 A. K. Vannucci, L. Alibabaei, M. D. Losego, J. J. Concepcion, B. Kalanyan, G. N. Parsons and T. J. Meyer, Proc. Natl. Acad. Sci. U. S. A., 2013, 110, 20918.

369 H. Baydoun, J. Burdick, B. Thapa, L. Wickramasinghe, D. Li, J. Niklas, O. G. Poluektov, H. B. Schlegel and C. N. Verani, Inorg. Chem., 2018, 57, 9748.

370 L. Wu, M. Eberhart, A. Nayak, M. K. Brennaman, B. Shan and T. J. Meyer, J. Am. Chem. Soc., 2018, 140, 15062.

371 R. M. Bullock, A. K. Das and A. M. Appel, Chem. - Eur. J., 2017, 23, 7626.

372 M. Yamamoto and K. Tanaka, ChemPlusChem, 2016, 81, 1028. 
373 W. Jiang, X. Yang, F. Li, Q. Zhang, S. Li, H. Tong, Y. Jiang and L. Xia, Chem. Commun., 2019, 55, 1414.

374 L. Duan, L. Tong, Y. Xu and L. Sun, Energy Environ. Sci., 2011, 4, 3296.

375 X. Liu, S. Inagaki and J. Gong, Angew. Chem., Int. Ed., 2016, 55, 14924.

376 K. L. Materna, R. H. Crabtree and G. W. Brudvig, Chem. Soc. Rev., 2017, 46, 6099.

377 L. Wu, A. Nayak, J. Shao and T. J. Meyer, Proc. Natl. Acad. Sci. U. S. A., 2019, 116, 11153.

378 T. Morikawa, S. Sato, K. Sekizawa, T. Arai and T. M. Suzuki, ChemSusChem, 2019, 12, 1807.

379 J. Li, W. Wan, C. A. Triana, Z. Novotny, J. Osterwalder, R. Erni and G. R. Patzke, J. Am. Chem. Soc., 2019, 141, 12839.

380 L. Xie, X. Li, B. Wang, J. Meng, H. Lei, W. Zhang and R. Cao, Angew. Chem., Int. Ed., 2019, 58, 18883.
381 P. Manna, J. Debgupta, S. Bose and S. K. Das, Angew. Chem., Int. Ed., 2016, 55, 2425.

382 B. A. Johnson, A. Bhunia and S. Ott, Dalton Trans., 2017, 46, 1382.

383 X. Liu, Y. Maegawa, Y. Goto, K. Hara and S. Inagaki, Angew. Chem., Int. Ed., 2016, 55, 7943.

384 X. Song, C. Peng and H. Fei, ACS Appl. Energy Mater., 2018, 1, 2446.

385 S. Gutiérrez-Tarriño, J. L. Olloqui-Sariego, J. J. Calvente, M. Palomino, G. Mínguez Espallargas, J. L. Jordá, F. Rey and P. Oña-Burgos, ACS Appl. Mater. Interfaces, 2019, 11, 46658.

386 N. Heidary, T. G. A. A. Harris, K. H. Ly and N. Kornienko, Physiol. Plant., 2019, 166, 460.

387 B. Wurster, D. Grumelli, D. Hötger, R. Gutzler and K. Kern, J. Am. Chem. Soc., 2016, 138, 3623.

388 Q. Shao, J. Yang and X. Huang, Chem. - Eur. J., 2018, 24, 15143. 